\title{
CHROMOSOME PAIRING AND CHIASMA FORMATION IN ALLOHEXAPLOID WHEAT, TRITICUM AESTIVUM ANALYZED BY SPREADING OF MEIOTIC NUCLEI
}

by

\author{
PREBEN BACH HOLM
}

Department of Physiology, Carlsberg Laboratory, Gamle Carlsberg Vej 10, DK-2500 Copenhagen Valby

\begin{abstract}
Keywords: Synaptonemal complex, allopolyploid, correction of pairing, prealignment, chromosome 5B
\end{abstract}

\begin{abstract}
An ultrastructural analysis of spread, silver stained meiotic nuclei of wheat, Triticum aestivum, has been performed. Partial tracings of lateral components and synaptonemal complexes of a number of leptotene and early zygotene nuclei, and complete tracings of eleven mid zygotene, seven late zygotene, twenty pachytene, twelve early diplotene, twenty-one mid diplotene and sixteen late diplotene nuclei have permitted the following observations and conclusions: 1) The chromosomes appear to be randomly distributed within the spread leptotene and early zygotene nuclei. 2) The complement length, i.e., the lateral component length of the three genomes, decreases from a mean of $2063 \mu \mathrm{m}$ at mid zygotene to $1806 \mu \mathrm{m}$ at late zygotene and $1474 \mu \mathrm{m}$ at pachytene. 3) Chromosome pairing is initiated preferentially from the telomeres. These are aggregated during zygotene whereby a chromosome bouquet is established. Synaptonemal complex formation is thereafter also initiated interstitially, the mean number of synaptonemal complex segments being 156 per nucleus in the eleven mid zygotene nuclei where on the average $61 \%$ of the complement is paired. 4) Chromosome pairing and synaptonemal complex formation remain frequently incomplete. In most cases, however, more than $95 \%$ of the complement has synapsed. 5) Interlocking of lateral components and synaptonemal complexes is frequent at zygotene. Detailed analysis of one mid zygotene nucleus revealed more than 20 interlockings, most of them being bivalent interlockings. At late zygotene there was a mean of 5 bivalent interlockings per nucleus and a few chromosome interlockings. Some interlockings persist up to pachytene and a few may remain into diplotene. 6) Multiple associations of chromosomes, probably signifying pairing between homoeologues, are seen in eight of the eleven mid zygotene nuclei, in four of the seven late zygotene nuclei, in three of the 20 pachytene nuclei and possibly in one of the early diplotene nuclei. 7) Synaptonemal complex degradation is initiated at a limited number of sites at the pachytene to diplotene transition, the number of retained synaptonemal complex segments amounting to 89,119 and 136 at early, mid and late diplotene. This exceeds thè number of chiasmata observable at metaphase I in the light microscope by a factor of two to three. 8) It is proposed that the diploid behaviour of allohexaploid wheat results from: I) A high stringency of chromosome pairing at the beginning of zygotene whereby pairing preferentially is initiated between homologues. II) Transformation of multiple association into bivalents at the end of zygotene and during pachytene before crossing over occurs and/or III) a suppression of crossing over between paired segments of homoeologous chromosomes. The possible effect of the Ph gene(s) on the long arm of chromosome 5B is discussed.
\end{abstract}

\section{INTRODUCTION}

The common bread wheat, Triticum aestivum is generally considered as a type example of an allopolyploid. Extensive investigations have revealed that it is an allohexaploid $(2 n=6 x=42)$ which appears to have arisen by two successive hybridization events followed by chromosome doubling involving three different 
species, (T. monococcum, $2 x=14$, A genome; unknown species, $2 x=14$, B genome; $T$. tauschii, $2 x=14$, D genome) (see 58, 59 for review). Breeding experiments and genetic and cytological studies have shown that each pair of chromosomes is partly homologous (homoeologous) to a chromosome pair in each of the two other genomes $(44,58,59)$. In spite of a close genetic relationship between the three genomes, crossing over and chiasma formation occur only between homologues and never between homoeologues, the outcome being disomic inheritance, an exclusive presence of bivalents at metaphase I and a regular segregation.

Crossing over and chiasma formation between the homoeologues of wheat do take place however, in F1 hybrids of rye or other wheat species with hexaploid wheat lacking particular chromosome arms, such as the short arm of chromosome $3 \mathrm{~A}$, the short arm of chromosome $3 \mathrm{D}$ or the long arm of chromosome $5 \mathrm{~B}$ (see 59 for review). These chromosome arms may thus contain genes affecting meiotic chromosome pairing and recombination. In the absence of one or more of these genes crossing over and chiasma formation can occur between the partly homologous chromosomes provided that there is no homologous pairing partner available, i.e., no competition by homologues.

In hexaploid wheat only the long arm of chromosome $5 \mathrm{~B}$ has proven to have an effect on recombination between homoeologues. In plants nullisomic for chromosome 5B or its long arm, crossing over occurs between homoeologous chromosomes $(50,54,55,57)$ : On the average each cell contained 1.4 univalents, 17.22 bivalents, 0.47 trivalents and 0.59 quadrivalents while pentavalents and hexavalents were rare. Several microsporocytes did not have multivalents, while others had many (54). More extensive chiasma formation betweeen homoeologues is observed in haploids lacking chromosome 5B (means of 3.17-4.17 bivalents and $0.86-2.00$ trivalents per cell) compared to the euhaploids where the complement at metaphase I consists almost exclusively of univalents (54, 55). WALL et al. $(64,65)$ obtained an ethyl methane sulfonate induced variant of wheat which in F1 hybrids with rye had a frequency of chiasmata between homoeologues approaching that observed in hybrids deficient for chromosome 5B. No effect was noted in hexaploid wheat homozygous for the mutation. The mutant phenotype segregated together with the long arm of chromosome 5B and behaved in crosses like a Mendelian recessive gene. It was concluded that the mutation had occurred in a single locus on the long arm of chromosome 5B. The normal dominant allele was designated $\mathrm{Ph}$ and its recessive allele ph (pairing homologous). A mutant gene termed $\mathrm{Phlb}$, located on the long arm of chromosome $5 \mathrm{~B}$, was subsequently identified in $\mathrm{x}$-rayed material (60). In F1 hybrids between this mutant line and tetraploid Triticum kotschyi, the frequency of trivalents and quadrivalents was drastically increased compared to $\mathrm{F} 1$ hybrids of normal wheat and $\mathrm{T}$. kotschyi and was even somewhat higher than that observed in hybrids between these two species lacking chromosome 5B. Even pentavalents were frequent (mean of 0.40 per cell). This mutant has later been used to transfer chromosome segments from Agropyron elongatum (61) and Secale cereale (42) into wheat, by crossing over between the homoeologues of the parental genomes. Supporting evidence for the presence of a $\mathrm{Ph}$ gene on the long arm of chromosome $5 \mathrm{~B}$ was obtained by showing that a deletion of a region between two C-bands on the long arm of chromosome 5B in tetraploid wheat, $\mathrm{T}$. turgidum, permitted chiasma formation between homoeologues in hybrids with tetraploid species of Aegilops (15).

A large number of speculations have been presented to explain the diploid-like behaviour of meiosis of allohexaploid wheat and other allopolyploids $(58,59,66)$. In wheat it is generally assumed that the gene(s) residing on the long arm of chromosome 5B suppresses pairing of homoeologues during zygotene $(58,59)$, possibly by affecting the location of the individual chromosomes during the premeiotic and preceding interphases $(17,18$, see section 4.7 for discussion). DRISCOLl et al. (14) proposed, however, in a mathematical study of the frequencies of the different chromosome configurations at metaphase $I$ in various lines of wheat and wheat hybrids that the degree of association within the individual groups of homoeologues were unaffected by the presence/absence of chromosome 
5B, but that the Ph gene(s) somehow "rendered sites for chiasma formation of homoeologues more similar". It was hypothesized $(12,13)$ that an enzyme system was affected that determines whether various types of pairing events proceed to chiasmata.

A major limitation to these studies and speculations on meiosis in allopolyploids, is that only chromosomes at metaphase I are amenable for light microscopic investigations. Any deductions as to the pairing behaviour of chromosomes during zygotene from observations at metaphase I therefore rest on the assumption that the chromosome associations at metaphase I constitute a true image of the initial chromosome pairing at zygotene. Extrapolating from this assumption the rather unfortunate term "pairing" is used to describe the chromosome configurations at metaphase I.

Ultrastructural studies of serially sectioned and spread zygotene and pachytene nuclei have shown that this assumption is incorrect. A major argument for a premeiotic alignment of homologues has been the virtual absence of interlocked chromosomes and bivalents at metaphase I. It is now evident from ultrastructural studies of zygotene nuclei that interlocking of chromosomes and bivalents is a regular feature of the zygotene chromosome pairing in all organisms (see 70 for review) where the zygotene stage has been analyzed. These interlockings are subsequently corrected by breakage-reunion of one or more of the interlocked or interlocking chromosomes. This breakage-reunion mechanism accounts for the rarity of interlockings at metaphase I. Secondly, observations from autopolyploid silkworms have shown that multivalent associations in the absence of crossing over are subjected to a correction process during pachytene whereby bivalents are formed at the expense of multivalents (53). A correction of translocation quadrivalents into heteromorphic bivalents in the absence of crossing over on both sides of the region of pairing partner exchanges has also been inferred from observations in man, mouse, and the basidiomycete Coprinus cinereus (see 70 for review).

A partial reconstruction of an early zygotene nucleus of euploid wheat (38) and a complete reconstruction of a late zygotene and a pachytene nucleus revealed the presence of multivalents and interlocking at zygotene, while only bivalents were present at pachytene (27). This showed that also in allopolyploids interlocking is a regular feature of the zygotene chromosome pairing and that correction of multivalents into bivalents is important for ensuring a disomic inheritance and regular segregation at the first meiotic division. It was proposed (27) that the Ph gene(s) might suppress crossing over until the correction of multivalents into bivalents was complete, while in the absence of chromosome 5B, crossing over occurs before the transformation of the multivalents. Subsequent work on allotriploid and allotetraploid Lolium, where chiasma formation between homoeologues can be modified by the presence of B chromosomes has supported this proposal by showing that multivalents formed during zygotene are corrected into bivalents and univalents in lines containing $\mathrm{B}$ chromosomes $(40,41$, see section 4.6 for discussion). As proposed by НовоLrH (27) correction of chromosome pairing may thus be a universal feature of chromosome pairing in allopolyploids.

A major problem in the analysis of chromosome pairing and chiasma formation and their genetic regulation in wheat is the amount of time required to reconstruct zygotene nuclei of wheat. It may thus take up to six months to reconstruct a single zygotene nucleus. Furthermore, complete reconstructions of the early and mid zygotene stages have not yet been possible due to the presence of knots of entangled lateral components and synaptonemal complexes in which the continuity of these structures often is lost $(38,71)$. It has therefore been considered desirable to try to employ on wheat microsporocytes a spreading technique $(2,3,22)$, where meiotic nuclei are subjected to a hypotonic shock in the presence of a weak ionic detergent, fixed and air dried onto a plastic membrane. This permits the analysis of the entire complement of synaptonemal complexes and lateral components in a two dimensional preparation. Although information on the three dimensional configuration of the nucleus as well as most of the details in the ultrastructure of the nucleus is lost, the technique is well suited for analysis of chromosome pairing. It was therefore decided to 
use this technique for analyzing chromosome pairing in a number of wheat genotypes with different numbers of copies of the long arm of chromosome 5B. Results from plants triisosomic for the long arm of chromosome $5 B$ have already been reported (71) and work on hexaploid plants with 0,1 and four copies of the long arm as well as work on haploids (triploids) with and without chromosome 5B are in progress. The present paper is addressed to chromosome pairing and chiasma formation in euploid wheat as revealed by spreading of meiotic nuclei.

\section{MATERIALS AND METHODS}

Plants of Triticum aestivum cv. Chinese Spring were grown at $20^{\circ} \mathrm{C}$ in a glasshouse at the Carlsberg Laboratory. During the spring and autumn the light period was prolonged by artificial illumination and during the wintertime the plants were grown under continuous light.

Spikes at the desired stage of development as judged by their size and thickness and the size of the individual spikelets were isolated. The three anthers of the most advanced floret of a spikelet from the middle of the spike were dissected out and transferred to a drop of ice cold maceration medium on a depression slide placed on a precooled block of aluminum. The maceration medium consisted of $120 \mathrm{~mm}(0.5 \%) \mathrm{NaCl}, 10$ mM-disodium ethylenediamine tetracetic acid (EDTA) and $6 \mathrm{~mm}$-sodium phosphate buffer, $\mathrm{pH}$ 7.4. The $\mathrm{pH}$ was adjusted to 7.5 by adding 1 $\mathrm{N}-\mathrm{NaOH}$. Following a measurement of the length of the anthers by an ocular micrometer, they were macerated with a needle and a scalpel blade and the suspension analyzed under phase contrast optics. The meiotic nuclei, liberated as a result of the anther maceration, could with some experience be staged precisely and it was thus not necessary to squash and stain one of the anthers for determination of stage.

Swelling of the nuclei was induced by adding two drops of an ice cold medium consisting of 10 mM-EDTA, 6 mM-sodium phosphate buffer, $\mathrm{pH}$ 7.4 , and $0.03 \%$ Tex-I, the $\mathrm{pH}$ being adjusted to 7.5 with $1 \mathrm{~N}-\mathrm{NaOH}$. Tex-I is a lemon-scented dishwashing agent from Blumøller A/S, Odense, Denmark and contains the two detergents sodium dodecyl benzene sulphonate and sodium dodecyl ether sulphate. The nuclei were allowed to swell for 1.5 min at $4{ }^{\circ} \mathrm{C}$, whereafter 6-7 drops of $4 \%$ formaldehyde containing $1.5 \%$ sucrose were added. The formaldehyde was prepared as a $40 \%$ stock solution by alkaline hydrolysis of paraformaldehyde at $70^{\circ} \mathrm{C}$, and the $\mathrm{pH}$ of the $4 \%$ solution was adjusted to 8.2 by adding 0.05 M-sodium tetraborate. After fixation for at least 30 min at $4{ }^{\circ} \mathrm{C}$, the suspension of anther debris and swollen mitotic and meiotic nuclei was poured onto slides covered with an Optilux membrane. Additional fixation mixture was added to wash down any remaining material sticking to the depression slide. The plastic membrane was prepared by dipping precleaned and polished slides into a $1 \%$ solution of Falcon Optilux petri dish plastic dissolved in redistilled chloroform. After drying, the edges of the slide were secured by painting with clear nail polish.

The suspension of anther material was air dried at room temperature overnight and if not considered sufficiently dry, placed on a hot plate at $60^{\circ} \mathrm{C}$ for a few hours the following day. The slides were then washed/ postfixed for $10 \mathrm{~min}$ in $1 \%$ glutaraldehyde in $0.5 \%$ Ficoll 400 (Pharmacia) with the $\mathrm{pH}$ adjusted to 8.2 with borate buffer, washed for $5 \mathrm{~min}$ in $0.5 \%$ Ficoll, $\mathrm{pH} 8.2$ and air dried at room temperature.

Silver staining of the slides was performed essentially as described by BLOOM and GoODPASTURE (8) and GoOdPasture and Bloom (24). The slides were incubated with a few drops of $50 \% \mathrm{AgNO}_{3}$ and placed on a hot plate at $60^{\circ}$ for $10 \mathrm{~min}$. The coverslips were then floated off, the slides washed in distilled water and air dried. Thereafter the slides were stained/developed in a 1:1 or 2:1 mixture of the following two solutions: 1) $4 \mathrm{~g}$ of silver nitrate in $5 \mathrm{ml}$ glass-distilled water and $5 \mathrm{ml}$ concentrated $\mathrm{NH}_{4} \mathrm{OH}$, the $\mathrm{pH}$ being adjusted to 11.3 with $\mathrm{HNO}_{3}$, and 2) a developer consisting of $1.2 \%$ formaldehyde which had been neutralized if necessary with sodium acetate crystals and thereafter adjusted to $\mathrm{pH}$ 5-6 with formic acid. The staining was monitored under phase contrast or conventional optics and, when the staining was considered appropriate, the coverslip was floated off and the slides washed in distilled water and dipped for 10 sec in $0.4 \%$ Kodak Photoflo 200 adjusted to $\mathrm{pH}$ 8.2 with borate buffer before air drying. Some 
spreads were stained with phosphotungstic acid (PTA) as described by Moses (46). One part of aqueous 4\% PTA was mixed with three parts of $96 \%$ ethanol and the slides were stained for 10 $\min$ in the mixture whereafter they were washed in $96 \%$ ethanol and air dried.

The stained slides were scanned by low magnification phase contrast optics. Well-spread and well-stained nuclei were marked by drawing a circle about $3 \mathrm{~mm}$ in diameter around them using an indelible felt tipped pen. Using a scalpel blade the membrane was scored along the edges and the membrane floated off on a clean water surface. Electron microscopic slot grids, previously dipped into an $8 \%$ solution of "Klab Op" in heptane (Klæb Op is a commercial glue for mounting photographs) were placed over the marked areas using a Baltzers vacuum tweezer, and the film picked up on a sheet of Parafilm. The grids were detached from the surrounding film before they were completely dry by scoring around the edges of the grid and placed in a grid box for further drying.

Zygotene and some pachytene nuclei were photographed at a negative magnification of 3,000 times and prints were made at a final magnification of 14,000 times. Usually 9-16 negatives were required to cover a nucleus. Some pachytene and all diplotene nuclei were photographed at a negative magnification of 1,000 to 2,000 times and were enlarged to a final magnification of 3,000 to 8,000 . The photographic montages of the zygotene and pachytene nuclei were covered with transparent plastic sheets and the individual lateral components traced onto them. The absolute length of the lateral components and synaptonemal complexes were measured with a map measurer.

\section{RESULTS}

\subsection{Early zygotene}

The fine structure of a spread nucleus at the leptotene-zygotene transition is shown in Figure 1. Lateral component formation appears to be complete or almost so and a distinct polarization of telomeres is apparent. A few of these have synapsed. Leptotene nuclei ranging in stage from the first appearance of segments of lateral components to a full leptotene stage, i.e., a stage where the lateral component formation appears to be completed, were also analyzed. At the earliest leptotene stages the lateral components are discontinuous and appear as a large number of individual pieces. Neither at this stage, nor at later stages of leptotene was there any indication of an alignment of long segments of lateral components.

A complete tracing of the lateral components at leptotene or at the beginning of zygotene was not attempted. As it can be seen from Figure 1 the lateral components can often be traced over longer distances but the large number of lateral component discontinuities as well as entanglement of lateral components do not appear to permit a tracing of the entire complement. In several regions short lateral component segments were aligned with another segment of lateral component, in particular in the region of aggregated telomeres where a few segments already have paired and formed a synaptonemal complex. In a few cases a lateral component appeared to be in the process of alignment with one partner in one region and with another lateral component in another region, indicating a potential future exchange of pairing partner. In other cases (Figure 1) the lateral components appeared to fold back and align with themselves.

A spread nucleus at a later stage of early zygotene is shown in Figure 2. Pairing is in progress but synaptonemal complex formation is largely confined to the distal segments of the chromosomes which form a typical bouquet. Complete tracings of nuclei at such an early stage of zygotene have likewise not yet been possible due to frequent discontinuities and entanglement of the lateral components. Exchanges of pairing partner between adjacent distal chromosome segments were not observed, but since the entire complement was not traced it cannot be excluded that some of the lateral components have synapsed with different partners at their two ends.

\subsection{Mid zygotene}

\subsubsection{General aspects}

Nuclei in which the degree of synapsis ranges from 25 percent to 75 percent are assigned to the mid zygotene stage. Eleven nuclei at this stage 
were traced. A survey micrograph of a spread mid zygotene nucleus (MZ5) is shown in Figure 3 and the complete tracing of this nucleus is presented as four separate drawings in Figure 4. The idiogram of this nucleus is shown in Figure 10.

In most of the spread nuclei a chromosome bouquet was present. The apparent absence of this organization in some nuclei may result from a different orientation of the nucleus on the membrane. If the aggregated telomeres were located in the top or the bottom half of the nucleus prior to drying down, the bouquet configuration will be difficult to recognize.

Complete tracing of lateral components in mid zygotene nuclei is not straightforward and requires that the analysis is performed on prints of a rather high magnification. Discontinuities and entanglements of lateral components and synaptonemal complexes are the major obstacles, and a large number of photographed spread nuclei had to be rejected due to too many ambiguities in the tracing. Entangled and overlapping lateral components could often be sorted out by analyzing these regions at 10-20,000 times magnification in the electron microscope provided that the lateral components were adequately stained. In several cases discontinuities of lateral components appeared to result from stretching of lateral components and synaptonemal complexes and often segments of extended lateral components were observed in such apparent gaps, permitting a tracing through this region. In other cases the lateral components were discontinuous without any apparent signs of a stretching. Several of these cases could be attributed to resolution of interlocking by breakage of lateral components (see section 3.2.2), the free ends still being located within the regions of presumptive interlocking. The remaining discontinuities of lateral components may reflect previous incidents of interlocking or a hooking of lateral components around other lateral components as well as breaks caused by proteolytic enzymes and nucleases liberated during the maceration of the tissue. Since in some nuclei the fragmentation is extensive and often occurs with some periodicity along the lateral component it is interpreted as a degradation. When maceration, swelling, and initial fixation were performed at low temperatures and in the presence of high concentrations of EDTA, the frequency of lateral component discontinuities was reduced supporting the above interpretation.

\subsubsection{Interlocking}

All the completely traced mid zygotene nuclei contained numerous interlockings of chromosomes and bivalents. In several cases in each nucleus one or both lateral components of an interstitial unpaired segment were stretched due to entrapment of one or more lateral components and synaptonemal complexes within the unpaired region (Figures 5 and 6). As also shown in Figure 4 several of the partially paired bivalents have large unpaired interstitial segments (e.g., bivalents 6 and 10 in Figure 4a, bivalents 3,9 and 11 in Figure $4 \mathrm{~b}$ and bivalents 2 and 4 in Figure 4c) which probably entrap a large number of chromosomes and bivalents. One or both lateral components are often irregularly bent and stretched where they appear to interlock other lateral components, e.g., bivalent 3 in Figure $4 \mathrm{~b}$. It was not possible to make a precise estimate of the total number of interlocking events in each nucleus due to difficulties in determining exactly the spatial relationship of lateral components as they cross each other in the flattened nucleus. This was in particular difficult for unpaired lateral components while it usually was possible to determine whether a lateral component is traversing below or above a synaptonemal complex. In order to obtain an estimate of the interlocking frequency, one nucleus (MZ10) which had relatively few lateral component discontinuities and several small unpaired segments, was examined in more detail. This nucleus contained at least 5 chromosome interlockings and at least 15 bivalent interlockings. In several cases one or more of the interlocked and interlocking lateral components showed indications of stretching and was longer than its homologous segment.

Interlocked or interlocking lateral components and synaptonemal complexes were often discontinuous, indicating breakage. Examples of discontinuities are shown in Figure $4 \mathrm{c}$ where one of the lateral components of bivalent 18 is discontinuous as it passes bivalent 2, in Figure 5 , 


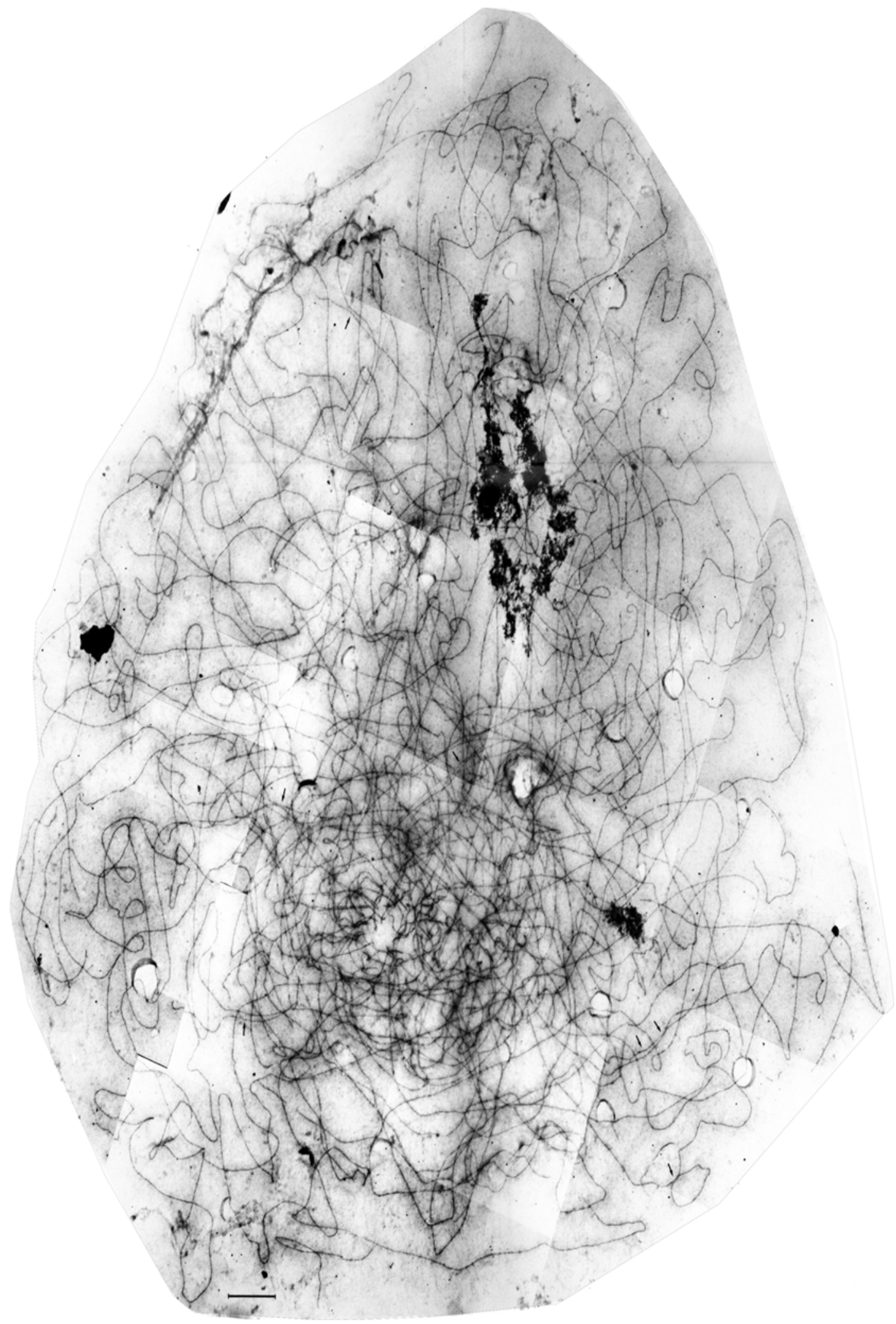

Figure 1. Survey micrograph of a spread nucleus at the beginning of zygotene. The few paired telomeres are aggregated in a small area. Aligned segments of lateral components are denoted by arrows. (Bar $=5.0 \mu \mathrm{m}$ ) 
where the continuity of one of the interlocking lateral components is almost lost where it is hooked around a synaptonemal complex and in Figure 7 where one of the unpaired lateral component is discontinuous in a region of presumptive interlocking (marked with diamond symbols). These discontinuities probably form as intermediates in the resolution of interlockings.

\subsubsection{Specificity and kinetics of chromosome pairing.}

The mean complement length, i.e., the lateral component length of the haploid complement, amounts to $2122 \pm 254 \mu \mathrm{m}$ (range 1869-2709 $\mu \mathrm{m}$ ) in the eleven mid zygotene nuclei, which on the average have $60.7 \%$ of the complement paired with a synaptonemal complex (range 45.0-71.6\%) (Table I). The nucleus (MZ3) with the longest complement length $(2709 \mu \mathrm{m})$ had clearly been subjected to stretching during drying and was irregularly elongated with often thin lateral components in contrast to the other ten mid zygotene nuclei which had an approximately circular outline. If the complement length of this nucleus is excluded the mean length of the mid zygotene nuclei amounts to $2063 \pm 171 \mu \mathrm{m}$ (range 1869-2392 $\mu \mathrm{m}$ ). As judged from the data presented in Table I there is no obvious correlation between complement length and degree of pairing.

Most chromosomes are at mid zygotene present as partially paired bivalents (Figure 10) and only few have engaged in multiple associations (see section 3.2.5). The central region of the synaptonemal complex could generally not be seen at this as well as the subsequent stages, but regions paired with a central region are easily distinguished from unpaired regions by the close alignment of the lateral components (Figure 7). Furthermore, the boundary between paired and unpaired segments is usually well demarcated by a pairing fork, where the lateral components diverge from the paired region.

The distribution of paired segments in the partially paired bivalents of the eleven mid zygotene nuclei is basically the same (Figure 10). Chromosome pairing and synaptonemal complex formation is usually complete in the distal segments of the bivalents, while several interstitial segments are unpaired. The mean number of synaptonemal complex segments per nucleus in the eleven nuclei is $156 \pm 38$ with a range from 114 to 228 (Table I). There is no apparent correlation between the degree of pairing and the number of synaptonemal complex segments. Thus, the nucleus with the lowest number of segments (MZ3, 114 segments) has as much of its complements paired as the nucleus with the largest number of segments (MZ10, 228 segments).

The length of the two lateral components in an unpaired interstitial region is nearly always the same. The measured length of the lateral components is shown in the idiograms in Figure 10. In cases where the two lateral components are of different length, the line symbolizing the shortest one is joined to the region representing the flanking paired segment by a line drawn at an oblique angle. The actual length of the lateral components is thus only represented by the length of the vertical lines. Only seven percent (76 out of 1099) of the unpaired interstitial regions where both lateral components are continuous had lateral components of significantly different length. Figure 8 illustrates a case where there is a major difference in length of the two lateral components in an unpaired region. This difference appears, however, to be compensated for by a corresponding difference in lateral components length in each of the two unpaired regions flanking this segment. Pairing in this distal segment thus appears to be between nonhomologous chromosome segments perhaps due to a presumptive chromosome interlocking involving the middle unpaired segment (Figure 8). In other cases, the differences in length of the lateral component segments of unpaired regions are more likely the result of mechanical stretching caused by interlocking or hookings. Since large unpaired interstitial regions may interlock several other chromosomes and bivalents an unequal mechanical stretching of the two lateral components is understandable.

In several of the unpaired chromosome regions one or occasionally both lateral components are discontinuous (Figure 10). As outlined in section 3.2.2 such breakages are often observed in regions of presumptive interlocking 


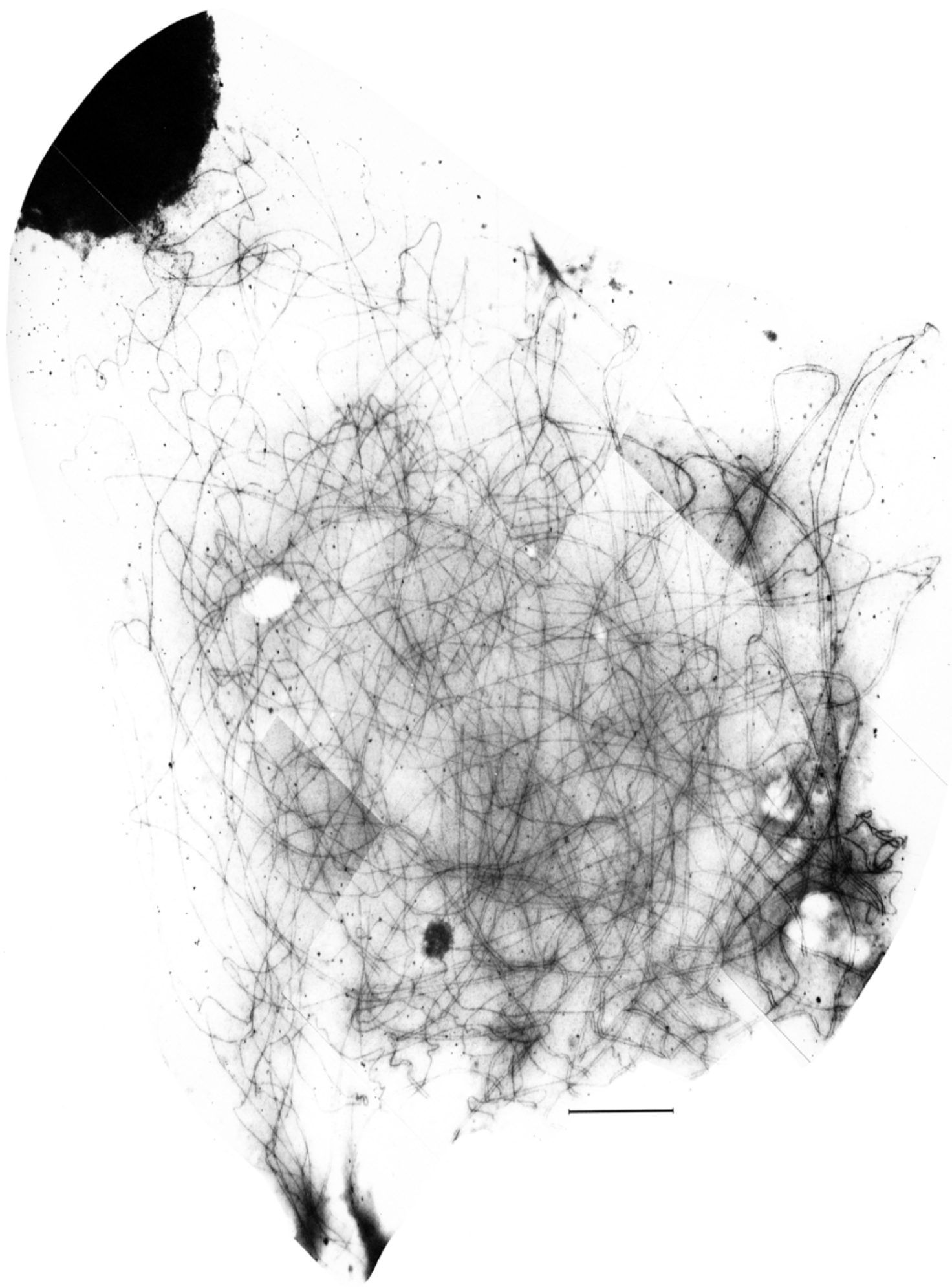

Figure 2. Survey micrograph of a spread early zygotene nucleus. The paired telomeres are clustered in the side of the rucleus opposite the nucleolus. (Bar $=5.0 \mu \mathrm{m}$ ). 
(Figure 7). In unpaired segments where only one lateral component is discontinuous, the continuous segment is generally somewhat longer than the combined length of the two segments of the broken one.

There are a few clearcut examples of nonhomologous pairing in the form of fold-back paired segments, i.e., in chromosome number 33 in nucleus $M Z 3$, in chromosome number 1 in nucleus $\mathrm{MZ4}$, in chromosome number 23 in MZ8 and in chromosome number 6 in MZ10 (Figure 10).

\subsubsection{The mid zygotene idiograms}

The partially paired bivalents and bivalent segments are in the idiograms arranged after decreasing length (Figure 10). Centromere-like structures as well as terminal knobs were occasionally found on some of the bivalents but their presence was too erratic to serve as structural markers for chromosome identification. Only the nucleolus organizing regions could be used as more reliable bivalent markers, but even these were often difficult to distinguish from other bivalent segments which were located below or above the nucleolus in the flattened nucleus. At mid zygotene there were never more than three presumptive nucleolus organizers, see section 3.3. A micrograph of two nucleolus organizing regions and the satellites is shown in Figure 9.

\subsubsection{The multiple associations at mid zygotene}

Multiple chromosome associations due to shift of pairing partner during synaptonemal complex formation were observed in eight of the eleven mid zygotene nuclei (Table I). This frequency must be regarded as a minimum estimate, since breakage of lateral components occasionally may have obviated the identification of additional multiple associations. Preliminary observations of spread zygotene nuclei from wheat lines nullisomic for chromosome $5 \mathrm{~B}$ and tetrasomic for chromosome 5D, which contain several multivalents, have shown, however, that multivalents are easily recognized using the present methodology and do not appear to be more susceptible to lateral compo- nent breakage than ordinary, partially paired, bivalents. Hence, it is considered unlikely that the actual frequency of multiple association is significantly higher than that observed. Partially paired bivalents with long unpaired regions where one lateral component is broken and somewhat shorter than the intact lateral component, might also have been involved in multiple association, e.g. bivalents 2 and 16 in nucleus MZ3, 1 and 2 in MZ4, 1 in MZ6, 1 in MZ7, and bivalent 5 in nucleus MZ8 (Figure 10). These gaps might also, however, have arisen by extensive stretching of the intact homologue or by loss of a lateral component segment. Such short segments are often seen in the flattened nucleus (Figure 10) apparently without contact to surrounding lateral components. A possible exception is bivalent 1 in nucleus MZ4, where the discontinuous lateral component is considerably longer than the intact lateral component, indicating that this bivalent has been released from a multiple association by breakage of lateral components.

Two different types of multiple associations can be recognized in the mid zygotene nuclei (Figure 10). The first type, Type I, is characterized by a single interstitial exchange of pairing partner per chromosome. Usually the pairing between the different combinations of lateral components extends from the presumptive telomere to approximately the middle of the lateral component. Examples of this type are the association of chromosomes A-E in nucleus $\mathrm{MZ1}$, of chomosomes $\mathrm{H}, \mathrm{I}$ and $\mathrm{J}$ in nucleus MZ4, and of chromosomes A-E in nucleus MZ6 (Figures 10, 11, 12 and 13). The second type, type II, are associations where a lateral component exchanges pairing partner over a short region and then switches back to its original partner. They thus appear to consist of apparently homologously paired bivalents, which are associated with a lateral component of another partially paired bivalent or a single lateral component through a short segment of synaptonemal complex (Figure 7). Examples of this type are the association of chromosomes F-K and L-N in nucleus MZ6, of chromosomes A-F and G-J in nucleus MZ7, of chromosomes A-C in nucleus $\mathrm{MZ8}$, of chromosomes A-D in nuclei MZ9 and MZ10 and of chromosomes A-D in 


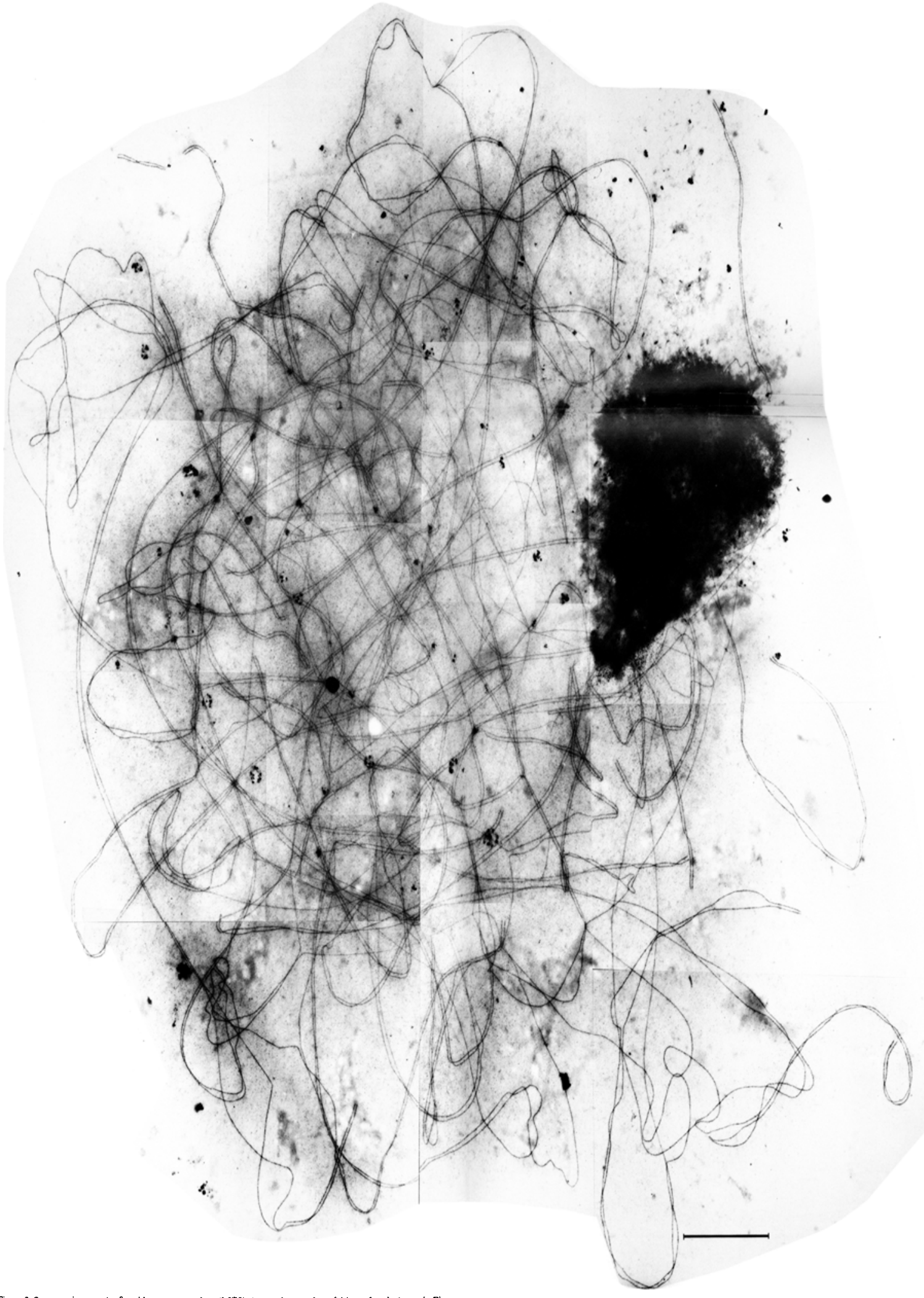

Figure 3. Survey micrograph of a mid zygotene nucleus (M25). A complete tracing of this nucleus is shown in Figure 4 and an idiogram of its complement in Figure $[0 .\langle\mathrm{Bar}=5.0 \mu \mathrm{m})$. 
P. B Holm Chromosome pairing in hexaploid wheat
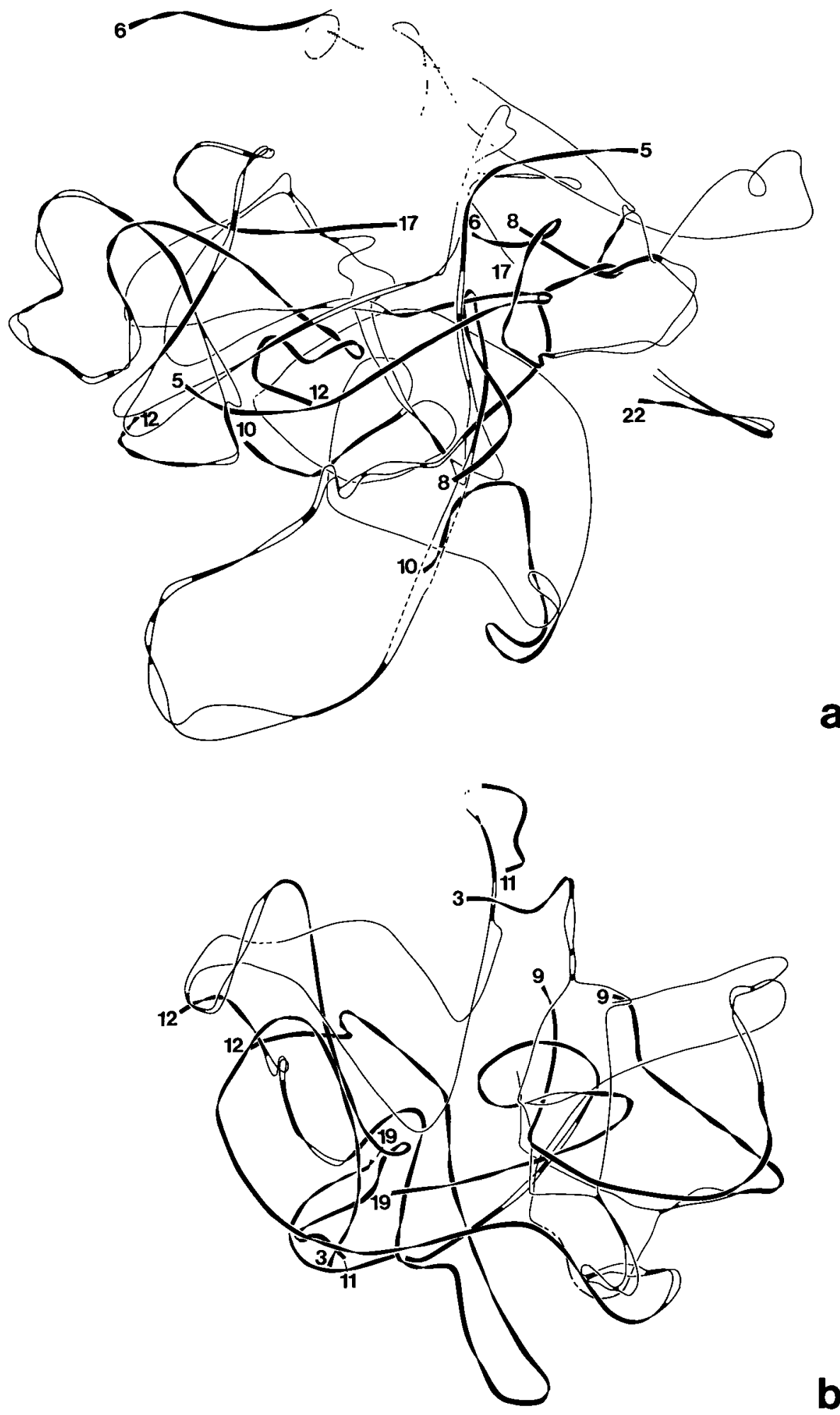

b 

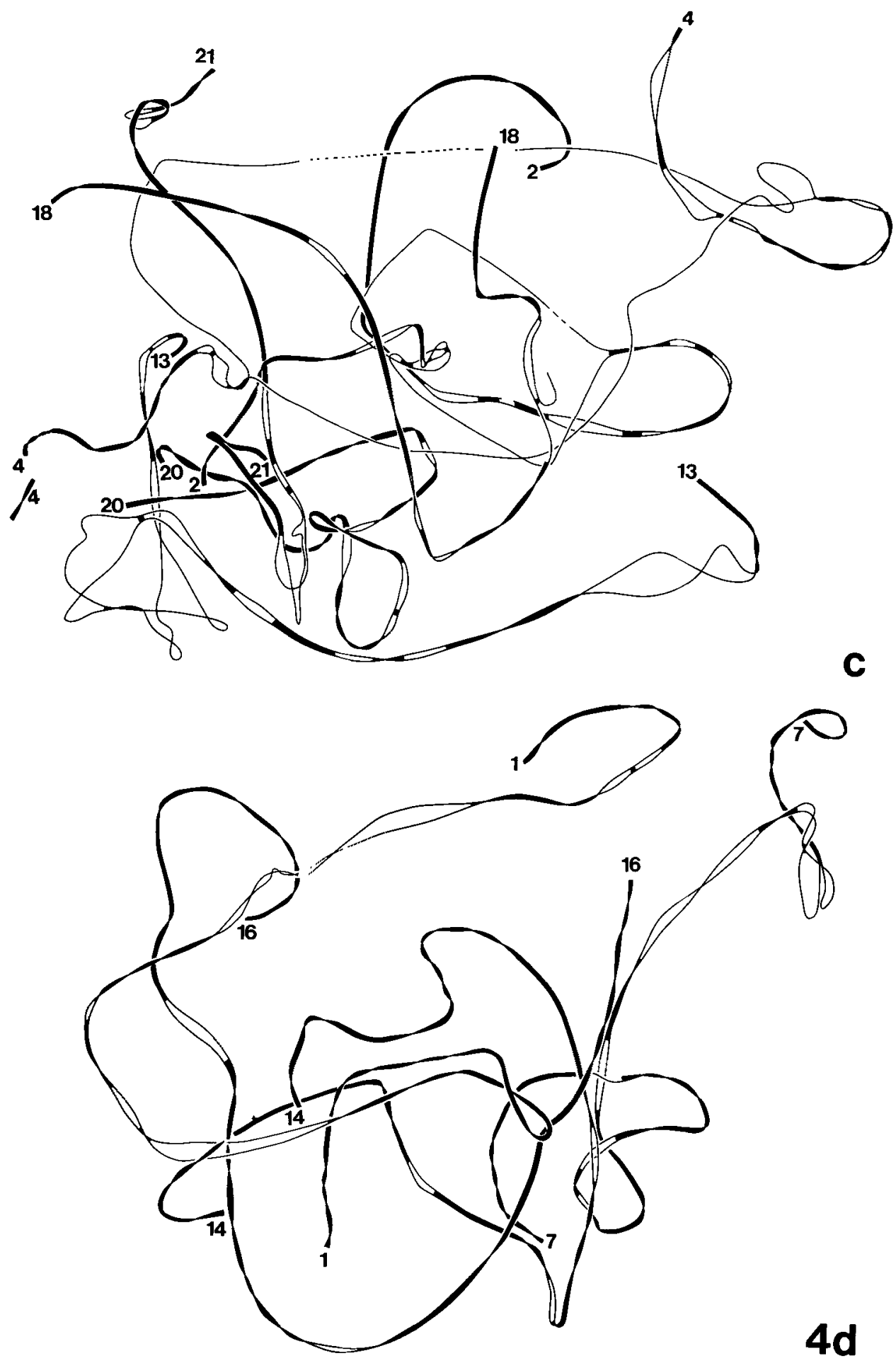
Figure 4. A complete tracing of a mid zygotene nucleus (MZ5). A survey micrograph of this nucleus is shown in Figure 3 and the idiogram of the complement is presented in Figure 10. The partially paired bivalents are numbered according to decreasing length. Stippled lines indicate discontinuities of the lateral components. The lines representing the lateral components are interrrupted where they cross each other. This is to facilitate tracing of the individual lateral component and does not reflect their spatial organization.

nucleus MZ12. More complex associations which appear to be mixtures of both types are represented by the association of chromosomes A-F in nucleus MZ4 and chromosomes O-T in nucleus MZ6.

A detailed characterization of these multiple associations is not possible since neither of the involved chromosomes can be identified. On the assumption that homoeologous chromosomes pair more readily than nonhomologous chromosomes, it is considered likely that a substantial fraction of the multiple associations, in particular associations of type I consists of homologous and homoeologous chromosomes and thus constitutes true multivalents.

\subsection{Late zygotene}

Idiograms of the seven completely traced late zygotene nuclei are shown in Figure 14. The mean length of the complement amounts at this stage to $1806 \pm 160 \mu \mathrm{m}$ with a range from 1641 $\mu \mathrm{m}$ to $2061 \mu \mathrm{m}$ (Table II). On the average $93 \%$ of the complement is paired with a synaptonemal complex, the degree of pairing ranging from 86 to $97 \%$. The mean number of synaptonemal complex segments per nucleus amounts to 45 and the unpaired segments are almost exclusively confined to interstitial parts of the bivalents. Most of these regions interlock other bivalents (Figure 15). It was estimated that there were 6, $7,1,6,2$ and 8 bivalent interlockings in the late zygotene nuclei LZ1, 2, 3, 4, 5 and 6, where between 93 and $97 \%$ of the complement had synapsed. Often the two lateral components interlocking other bivalents were of different length or one of the lateral components was broken. Presumptive chromosome interlockings were seen in the nuclei $L Z 1, L Z 2$ and LZ6. Breakage of both lateral components in an unpaired region, giving rise to two half bivalents appeared in several cases to result from previous interlocking events, since one or more synaptonemal complexes often were present between the unpaired ends of the two broken lateral components. The multiple associations often appeared to interlock several lateral components and synaptonemal complexes. As described below the associations predominantly involved six lateral components which form a six armed structure that encloses several other chromosomes.

At this, as well as at the pachytene stage, the nucleolus organizing regions were easier to identify than at mid zygotene. Three of the nuclei contained two while the last four nuclei contained three of these regions, located distally in the longer bivalents (Figures 9, 14). In the cases where only two nucleolus organizing regions are present they probably represent the ones in bivalents $1 \mathrm{~B}$ and $6 \mathrm{~B}$ which together contain $90 \%$ of the ribosomal cistrons, while the remaining 10 percent are located on chromosome 5D (4).

Four of the seven nuclei contained one or more multiple associations (Figure 14), whereas in the three remaining nuclei pairing is exclu-

Figure 5. A micrograph of an interlocking at mid zygotene and a tracing of the interlocking lateral components. A partially paired bivalent on the right side in the micrograph (denoted by arrowheads) appears to be interlocked between the lateral components presented as dotted lines. On the left side in the micrograph the other lateral component of the traced bivalent appears to interlock two or more bivalents. This lateral component is considerably longer than the homologous lateral component and its continuity is almost lost where it is hooked around a synaptonemal complex (denoted by arrows). (Bar $=1.0 \mu \mathrm{m})$. 

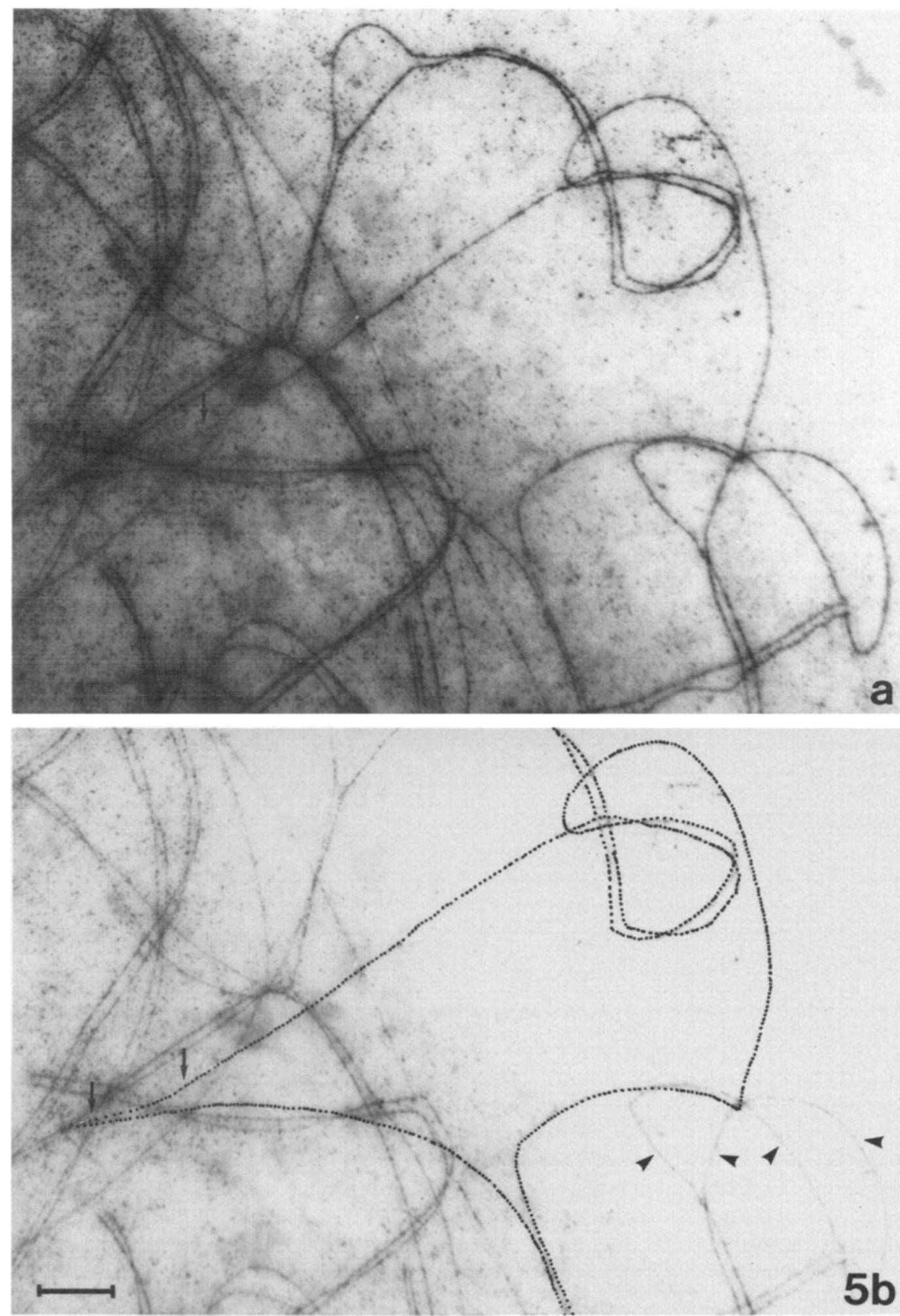

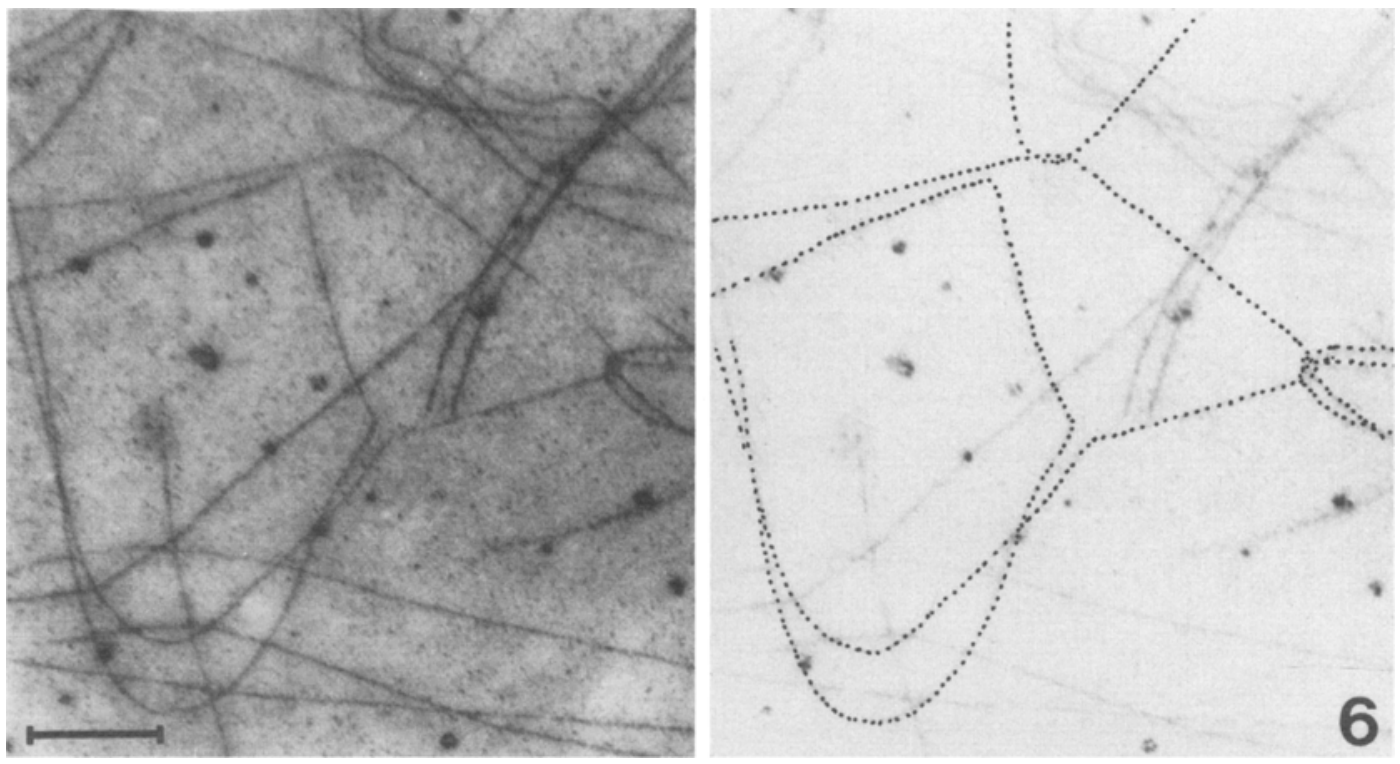

Figure 6. A micrograph and a tracing of an interlocking at mid zygotene. An unpaired lateral component (top of micrograph) and a synaptonemal complex (right side of micrograph) are trapped between two partially paired lateral components. One of the interlocking lateral components is longer than the other and appears stretched. (Bar $=1.0 \mu \mathrm{m}$ ).

\section{Table I.}

Mean complement length (half the lateral component length), degree of pairing in percent, number of synaptonemal complex (SC) segments and the number of chromosomes or chromosome fragments involved in multiple associations in eleven mid zygotene nuclei.

s.d., standard deviation

\begin{tabular}{lcccc}
\hline $\begin{array}{l}\text { Nucleus } \\
\text { number }\end{array}$ & $\begin{array}{l}\text { Complement } \\
\text { length }(\mu \mathrm{m})\end{array}$ & $\begin{array}{l}\text { Pairing } \\
\text { percent }\end{array}$ & $\begin{array}{c}\text { Number of } \\
\text { SC } \\
\text { segments }\end{array}$ & $\begin{array}{l}\text { Number of chromosomes } \\
\text { or chromosome fragments } \\
\text { in multiple associations }\end{array}$ \\
\hline MZ1 & 2173 & 65.4 & 140 & 6 \\
MZ2 & 2075 & 64.7 & 146 & 0 \\
MZ3 & $2709^{\mathrm{a}}$ & 71.6 & 114 & 0 \\
MZ4 & 2055 & 55.3 & 120 & 10 \\
MZ5 & 1869 & 64.9 & 134 & 0 \\
MZ6 & 2392 & 52.1 & 148 & $5,6,3,5$ \\
MZ7 & 2275 & 45.0 & 197 & 6,4 \\
MZ8 & 1926 & 58.1 & 207 & 4 \\
MZ9 & 2031 & 56.9 & 157 & 4 \\
MZ10 & 1944 & 69.7 & 228 & 4 \\
MZ12 & 1896 & 63.6 & 120 & \\
\hline Mean & 2063 & 60.7 & 156 & \\
\pm s.d. & \pm 171 & \pm 8.0 & \pm 38 &
\end{tabular}

a) The length is not included in the calculation of the mean due to extensive stretching of the lateral components of the nucleus. 


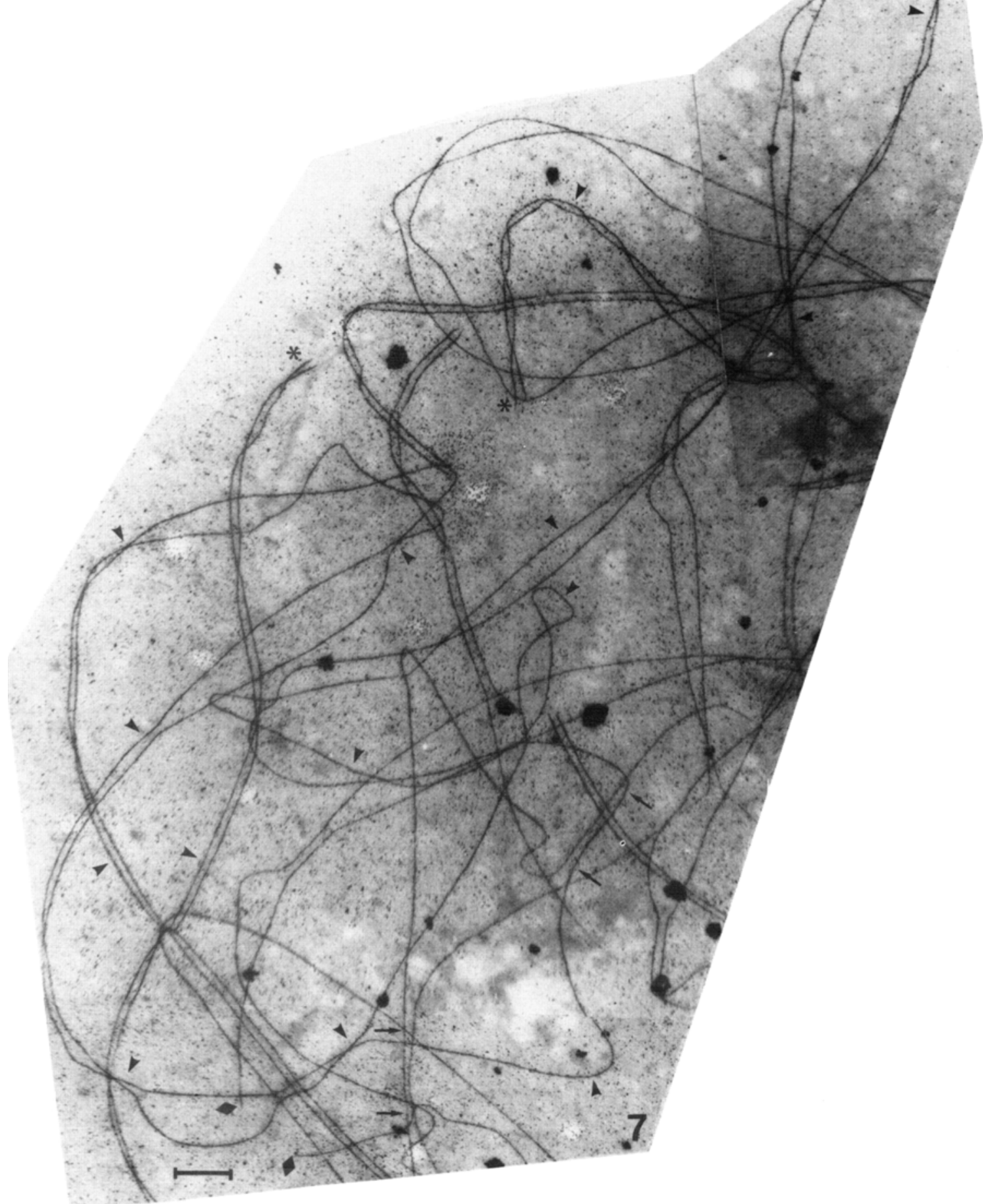

Figure 7. Micrograph of an entire parially paired bivalent from a mid zygotene nucleus. The lateral components of this bivalent are marked by arrowheads and its telomeres with asterisks. One of the lateral components is paired over a short distance with another lateral component (delimited by arrows) which at the bottom of the micrograph has engaged in pairing with a third lateral component (delimited by arrows). A break in a lateral component of a partially paired bivalent which appears to interlock the bivalent marked by arrowheads is denoted by diamond symbols. $($ Bar $=1.0 \mu \mathrm{m})$. 


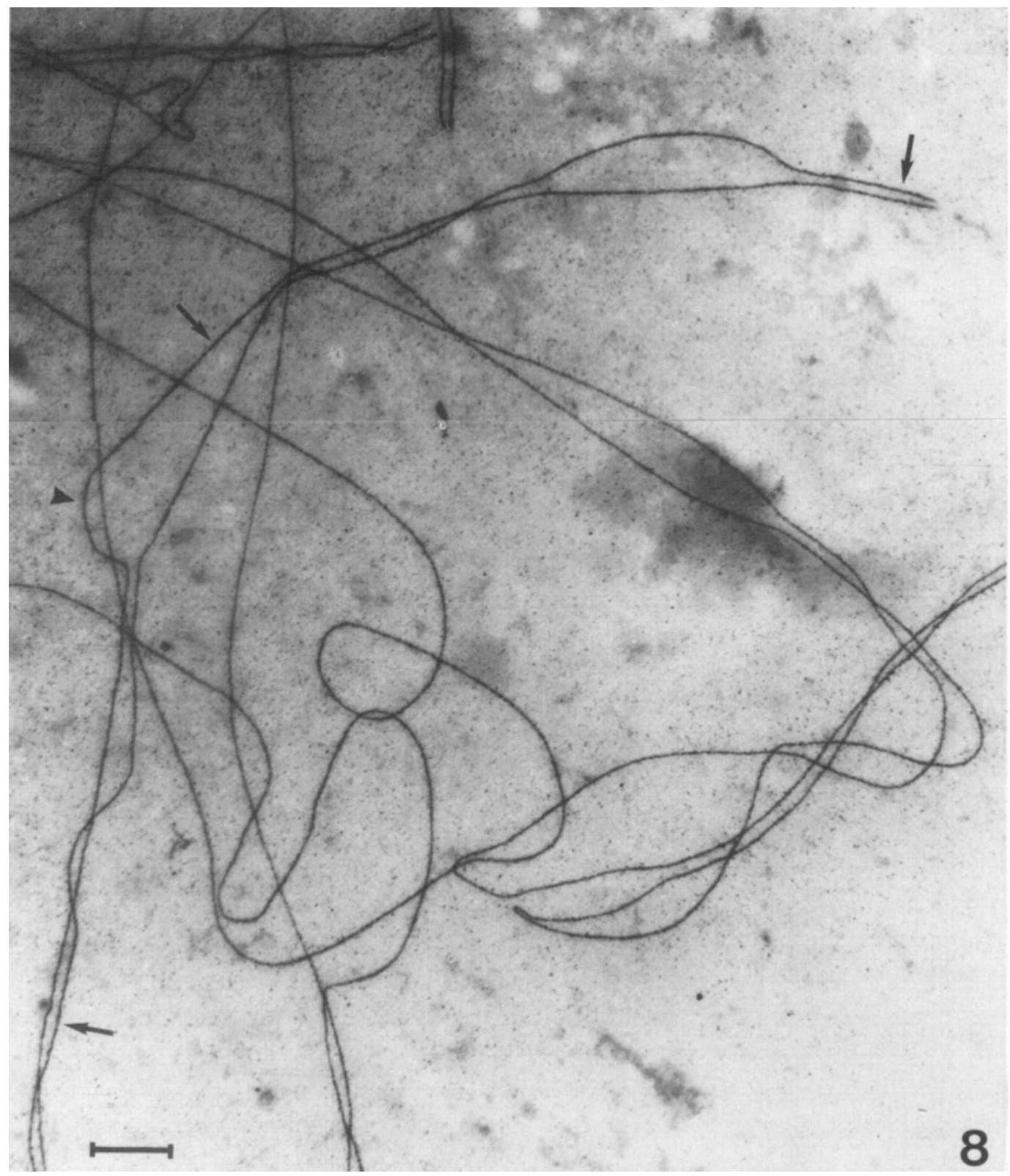

Figure 8. A micrograph of a partially paired bivalent (denoted by arrows) where the two lateral components are misaligned. A presumptive chromosome interlocking is denoted by an arrowhead. $(\mathrm{Bar}=1.0 \mu \mathrm{m})$.

sively in the form of nearly completely paired bivalents. As at mid zygotene, breakage of lateral components may have resolved additional multiple associations and the observed frequency thus represents a minimum estimate. In each nucleus there is a number of "half bivalents" of which a few may have been part of multivalents. There is, however, no indication that the number of multiple associations is significantly higher than that observed.

Tracings of the five multiple associations in the four nuclei are shown in Figure 17 and the 

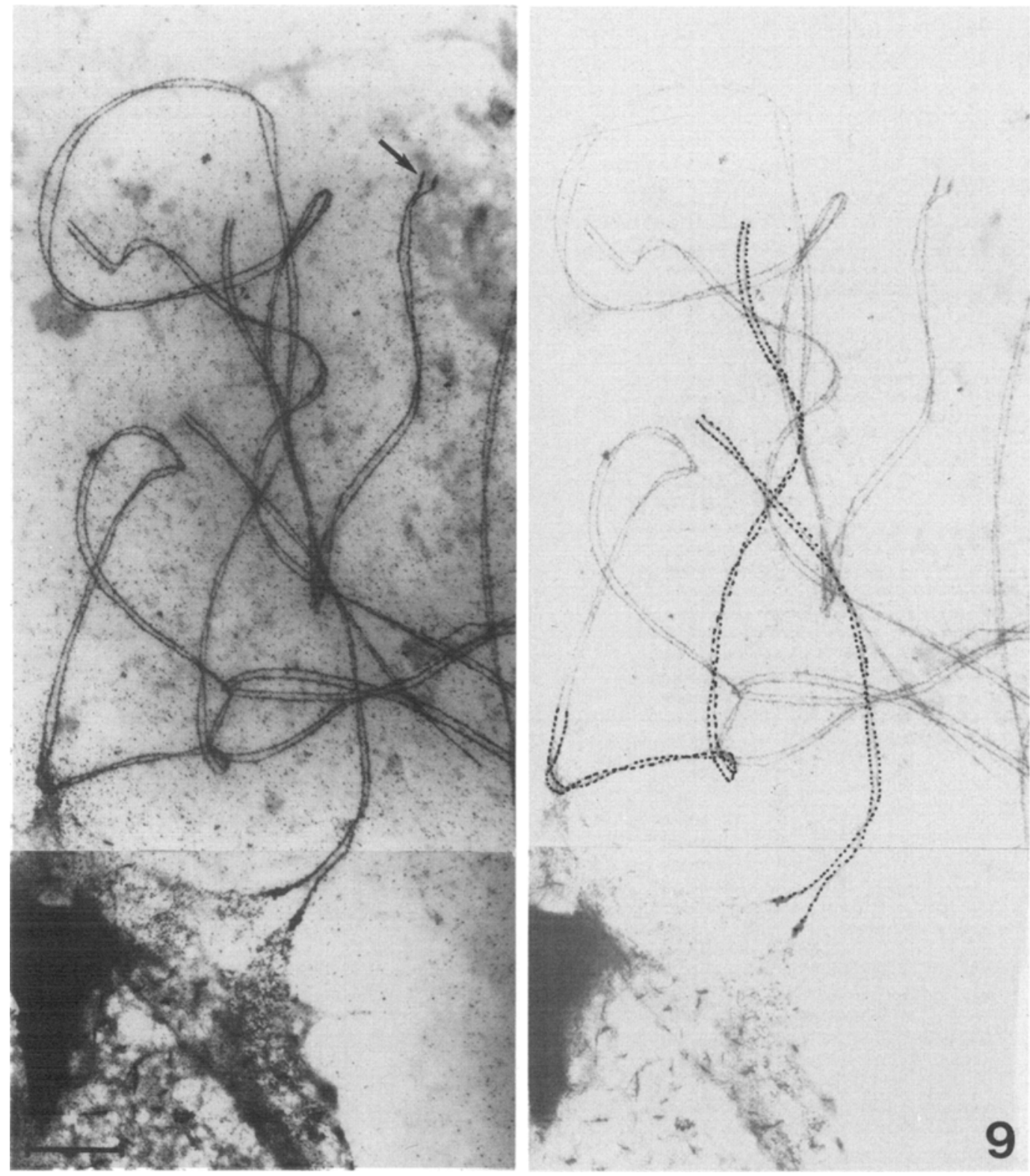

Figure 9. Micrographs of two nucleolus organizing regions of a pachytene nucleus with tracings of the satellites. One of the organizer bivalents is broken in the middle of the nucleolus organizing regions. The presumed free end of the organizing region which contains thickened lateral components is marked with an arrow. (Bar $=2.0 \mu \mathrm{m}$ ).

fine structure of one region of pairing partner exchange in Figure 16. In nuclei LZ2, 3, 4, 5 and 7 the multiple associations were of type $I$, consisting of five apparently intact lateral components and two fragments. It is considered likely that these associations are hexavalents each involving three pairs of homoeologues. In nucleus LZ5 the association of 12 chromosomes may have arisen by formation of a short nonhomologous synaptonemal complex segment between an association of six lateral components, possibly a hexavalent and an association of five 


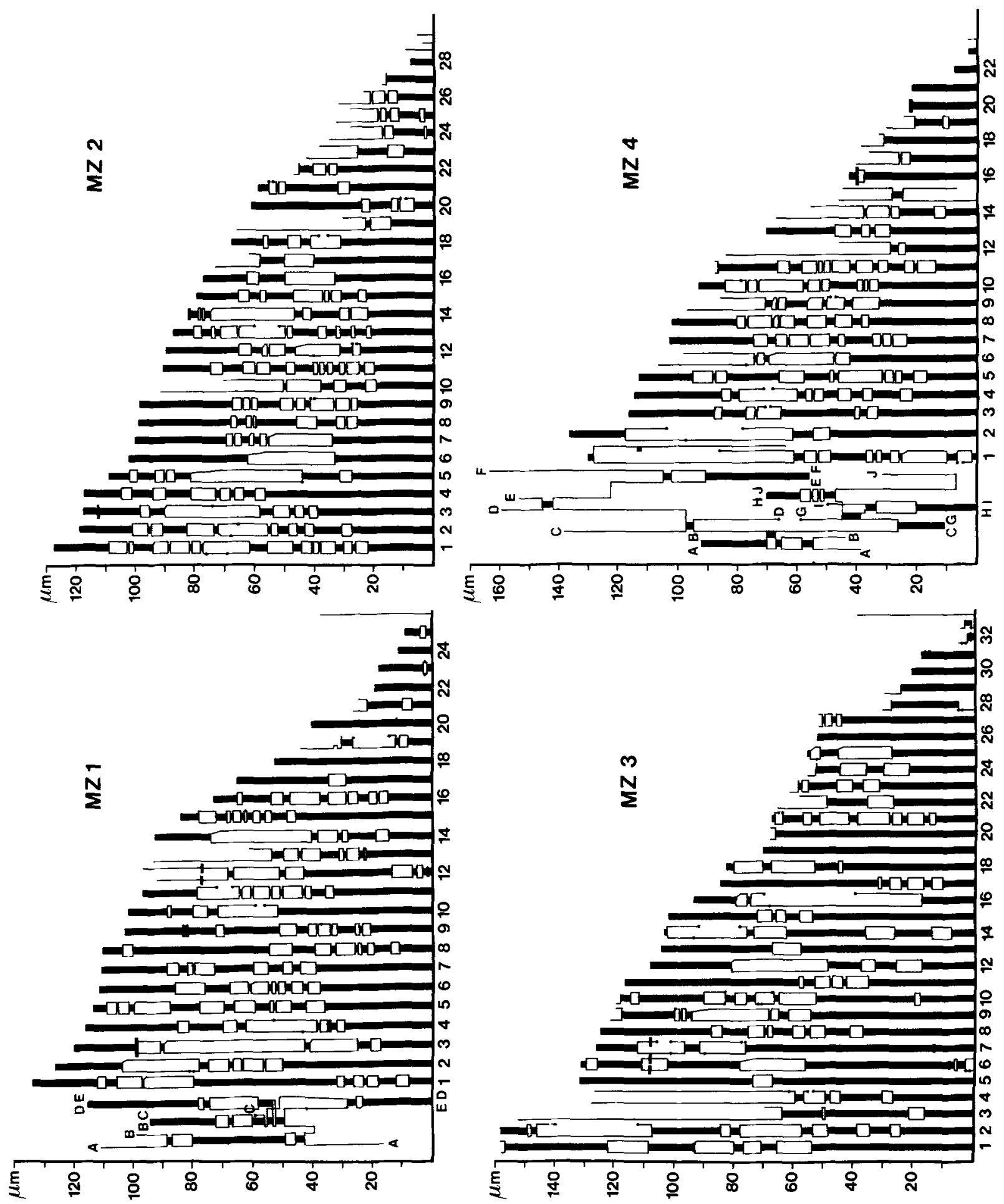




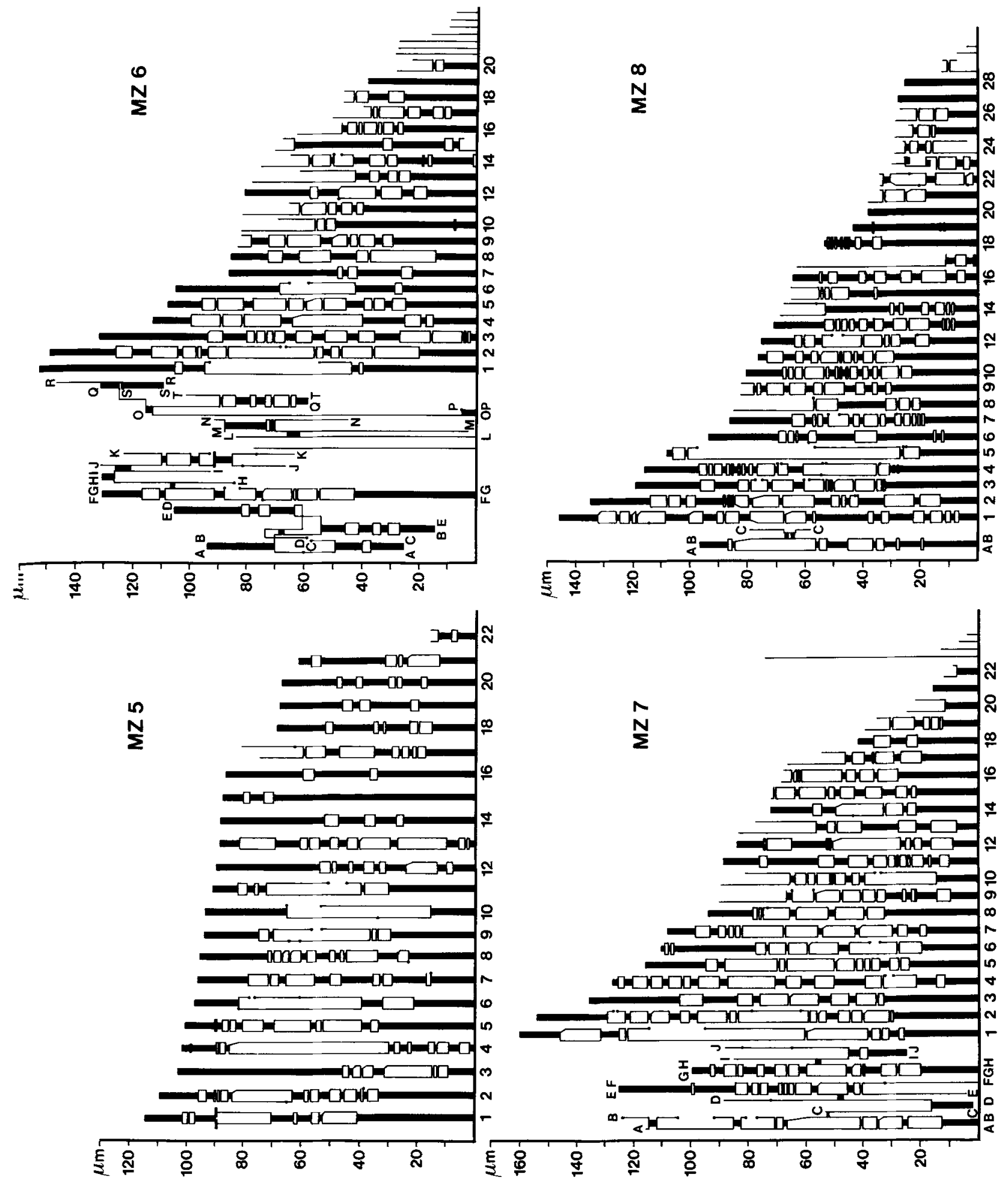



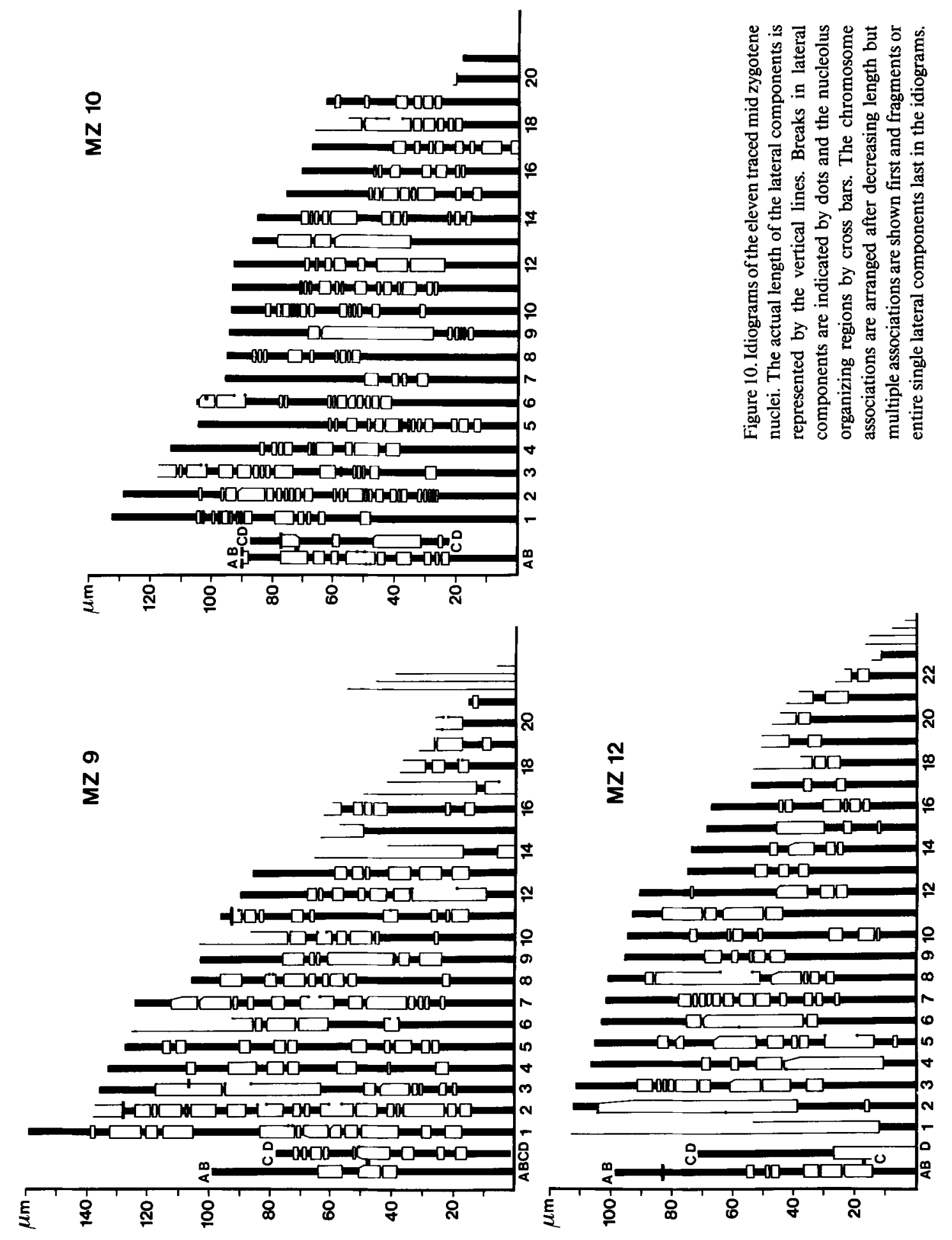


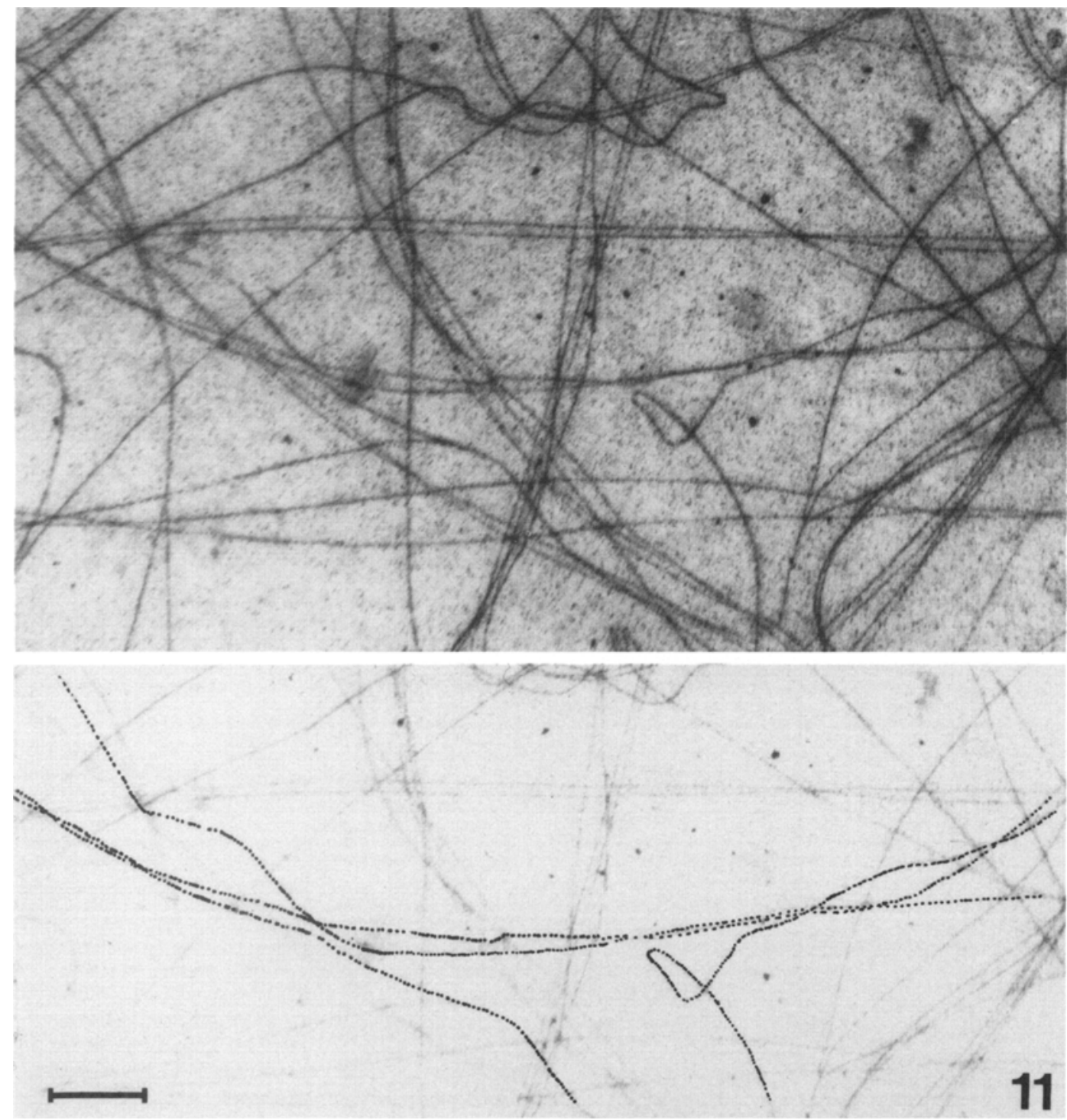

Figure 11. A micrograph and a tracing of a pairing partner exchange in a mid zygotene nucleus (nucleus number MZ4). The entire multiple association is shown in Figure 13c. $($ Bar $=1.0 \mu \mathrm{m})$.

lateral components. This association involves two of the nucleolus organizing chromosomes. In all these associations there is a regular alignment and synaptonemal complex formation of the two lateral components in the paired segments except in a single case (chromosomes AB in nucleus LZ4, Figure 17b) where two lateral component segments interlock a synaptonemal complex segment (Figure 15). In three of the nuclei, LZ3, LZ4 and LZ6 short unpaired regions have folded back and paired with themselves.

\subsection{Pachytene}

The transition from zygotene to pachytene in wheat spermatocytes is marked by a change in the outline of the flattened nuclei. While the 

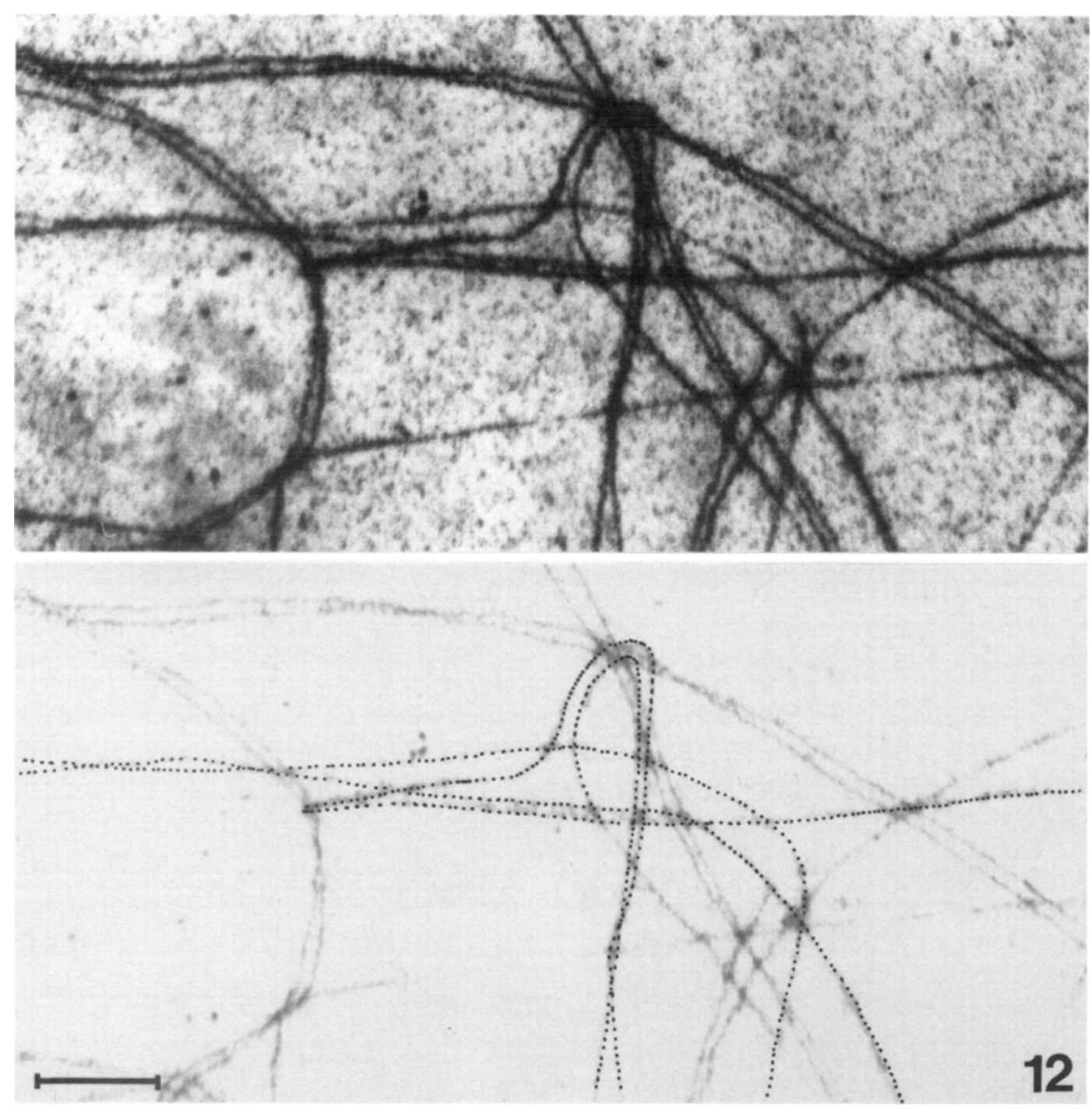

Figure 12. A micrograph and a tracing of a pairing partner exchange in a mid zygotene nucleus (nucleus number $\mathrm{MZ1})$. The entire multiple association is shown in Figure 13a. $(\operatorname{Bar}=1.0 \mu \mathrm{m})$.

spherical zygotene nuclei at all stages possess a bouquet configuration due to an aggregation of the attachment sites for the telomeres, pachytene nuclei appear to have a more even distribution of telomeres and the collapsed nuclei are generally elongated, often with the shape of a dumbbell (Figure 18). Occasionally the flattened nucleus has three lobes, but the individual bivalents generally span two or all three lobes. The changed organization of the nucleus is accompanied by a reduction in complement length from a mean of $1806 \mu \mathrm{m}$ at late zygotene to a mean of $1474 \pm 214 \mu \mathrm{m}$ (range 1194-1972 $\mu \mathrm{m})$ at pachytene (Table III). Chromosome pairing and synaptonemal complex formation seldom reaches completion. The mean degree of pairing in 19 of the 20 analyzed nuclei was $96.7 \%$. These nuclei were, however, selected for a nearly complete pairing and do not represent a random sample. Several nuclei were found 
which possessed the elongated shape and/or the short complement length characteristic of pachytene nuclei but where several segments were unpaired. One of these nuclei (P4, Table III) had only $65 \%$ of its complement paired. Additional evidence for incomplete pairing at pachytene is provided by the observation that early diplotene nuclei often have unpaired lateral component segments (see section 3.5), a phenomenon not normally encountered in other analyzed species (70).

The idiogram of a pachytene nucleus (nucleus P1) in Figure 19 illustrates that as in the late zygotene nuclei, the unpaired segments are interstitial, relatively short and present on several of the bivalents. Among the 20 pachytene nuclei there are, however, also nuclei where only one or two longer lateral component segments remain unpaired. Fold-back pairing is frequent in these segments. In several cases the lateral components of the unpaired segments appear to interlock other bivalents (Figure 20), while chromosome interlockings are seen seldomly.

Multiple associations were observed in three of the 20 analyzed pachytene nuclei. Nucleus P1 1 contained two type I associations of three lateral components of which one is shown in Figure 21, nucleus P18 contained a type II association of five lateral component segments and nucleus P20 two type I associations of three lateral components. As is the case at mid and late zygotene, this frequency is a minimum estimate since breakage of lateral components in unpaired regions might in rare cases obviate the identification of additional multiple associations.

\subsection{Diplotene}

The initiation of synaptonemal complex degradation marks the transition from pachytene to diplotene. The degradation is initiated at a relatively low number of sites generating a number of discrete synaptonemal complex segments, which have retained their normal morphology (Figure 22). In the 12 analyzed early diplotene nuclei the mean number of segments is $89 \pm 21$ with a range of $44-118$. The combined length of these segments is $946 \mu \mathrm{m}$ (Table IV). In some of the nuclei (e.g., numbers ED 3, 10, 11 and 12) no, or very few, unpaired lateral components are present, while in other nuclei unpaired lateral components were abundant. A substantial part of the complement has thus been unpaired at the beginning of early diplotene. As shown in Figure 23, some of these nuclei exhibit the circular outline characteristic for spread zygotene nuclei, indicating that such nuclei have progressed directly from zygotene to diplotene. The mean length of unpaired segments amounts to $162 \mu \mathrm{m}$ and the complement length to $1108 \mu \mathrm{m}$. On the assumption that paired and unpaired segments are not differentially degraded, $85 \%$ of the complement has been paired with a synaptonemal complex, when the degradation is initiated.

The lack of synaptonemal complex and lateral component continuity over longer distances represents major obstacles to the identification of interlockings and multiple associations. In a few cases presumptive interlockings have been seen, a possible example is shown in Figure 24. Multiple associations appeared to be rare or entirely absent. When analyzed at low magnification a number of such apparent associations were seen, but at higher magnifications they all turned out to be either entangled lateral components, fold-back paired segments, or presumptive interlockings. Only one case of a possible multiple association was found (Figure 25) at this as well as at the following stages.

At mid diplotene the unpaired lateral components are almost completely eliminated. Only lateral components of a mean combined length of $38 \mu \mathrm{m}$ per nucleus remain (Table V). Degradation of the synaptonemal complex segments appears primarily to affect the ends of the segments reducing their length between early and mid diplotene from $946 \mu \mathrm{m}$ to $647 \mu \mathrm{m}$. In the same period the number of segments increased from 89 to 119 due to interstitial degradation. The synaptonemal complex segments still combine the homologous chromosomes as evidenced by the ultrastructure of insufficiently spread bivalents. As illustrated in Figure 26 individual bivalents and bivalent segments can be identified in such nuclei, consisting of a central synaptonemal complex segment, surrounded by chromatin-like fibers. Interlockings and multiple associations were not observed at this stage. 

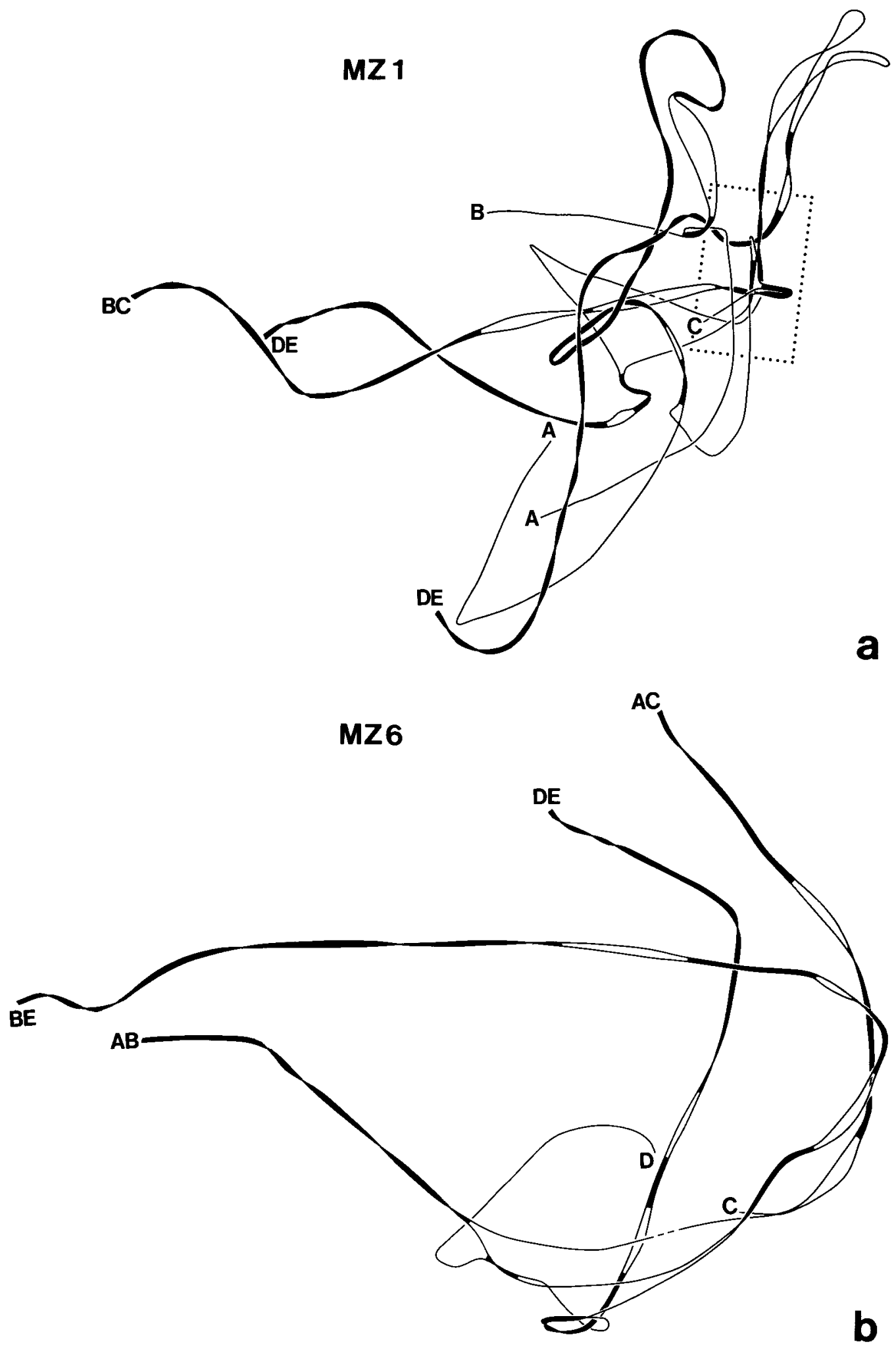


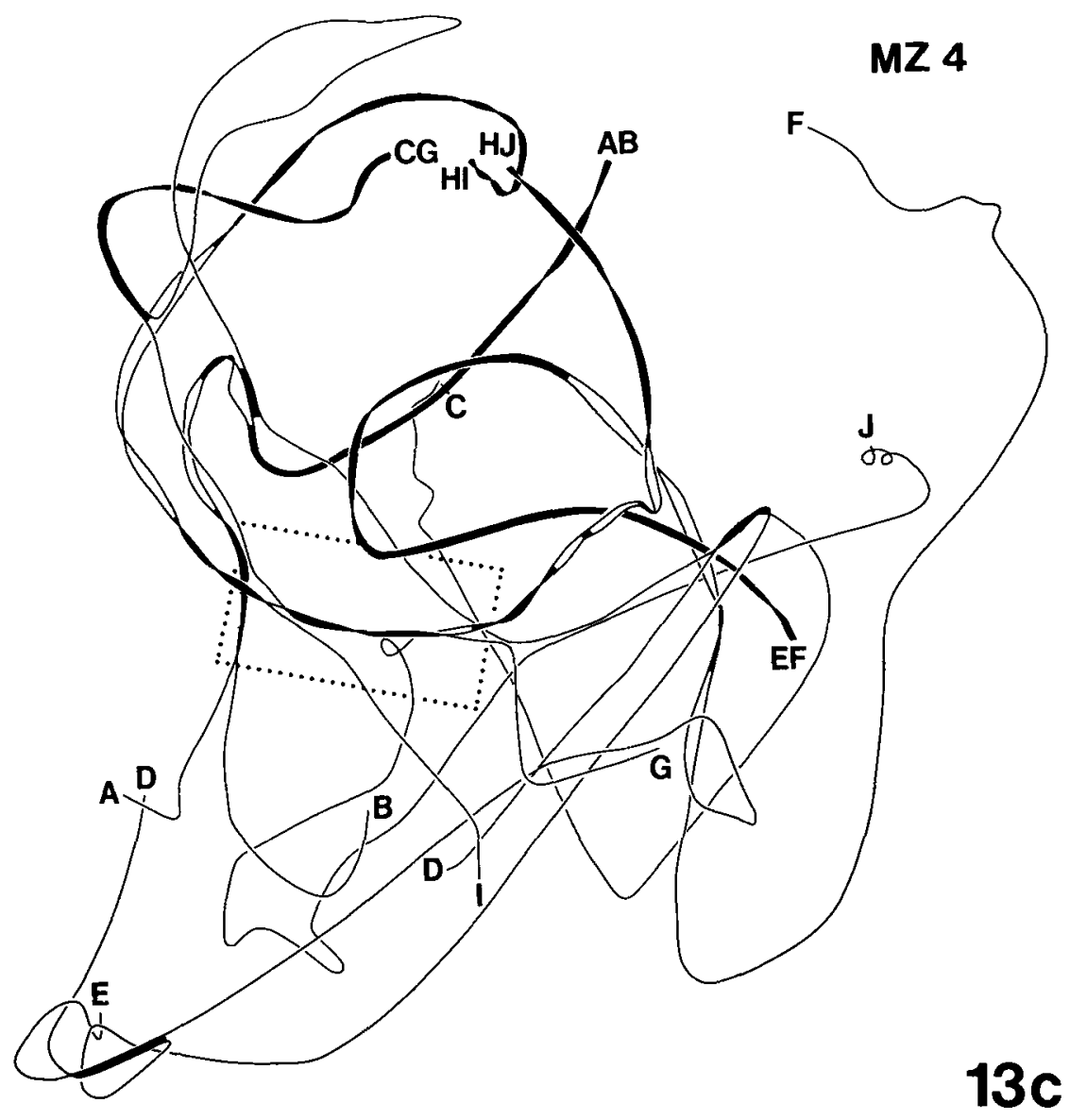

Figure 13. Tracings of multivalents observed in mid zygotene nuclei. The numbers on the tracings refer to the numbers of the nuclei and the letters denoting the individual chromosomes correspond to those used in the idiograms in Figure 10. Micrographs of the marked areas in Figures 13a and $13 \mathrm{c}$ are shown in Figures 11 and 12.

The last stage where synaptonemal complex segments can be identified is late diplotene. Spread diakinesis nuclei, i.e., nuclei where chromatin condensation has commenced, have also been identified, and these nuclei are devoid of synaptonemal complex remnants. The late diplotene nuclei are extensively spread with a homogeneous and electron transparent nuclear content save the synaptonemal complex segments (Figure 27). Some segments are relatively indistinct or with a morphology like that at pachytene, while others had thickened lateral components (Figure 28). In some cases the lateral components nearly merge, filling the central region (Figure 29). The segments within a nucleus appeared to display a continuous variation in thickness of the modified lateral components and a classification into different types was not considered possible.

The data in Table VI reveal that the complement length has decreased to half the mid diplotene length ( $325 \mu \mathrm{m}$ versus $647 \mu \mathrm{m})$ and that unpaired lateral components now are completely eliminated. In some cases unpaired ends of lateral components protrude from the end of these segments, but these minor lateral component segments are not measured. As is the case at the previous stages the degradation of the synaptonemal complex segments occurs primarily from the ends of the segments. The 

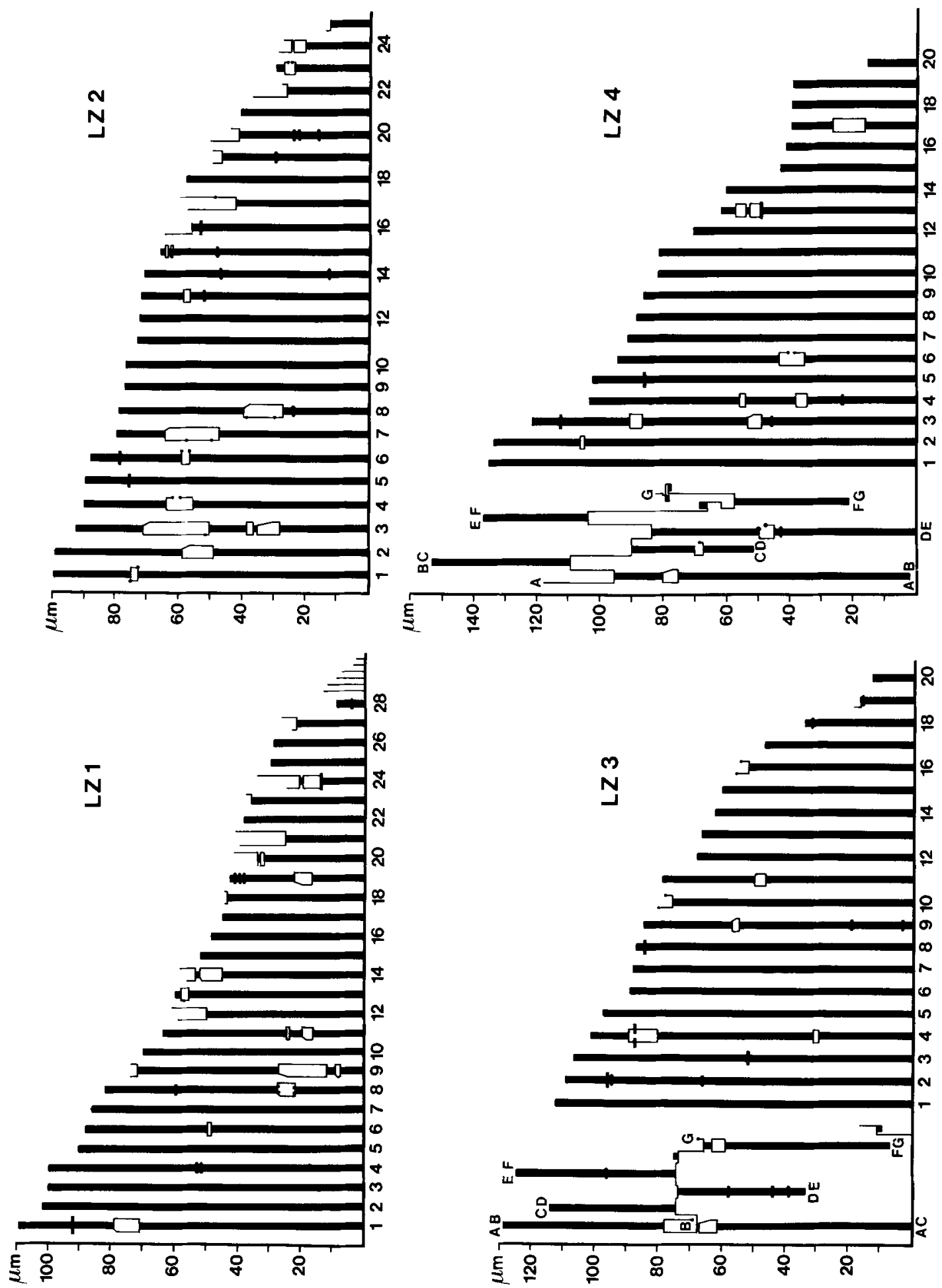

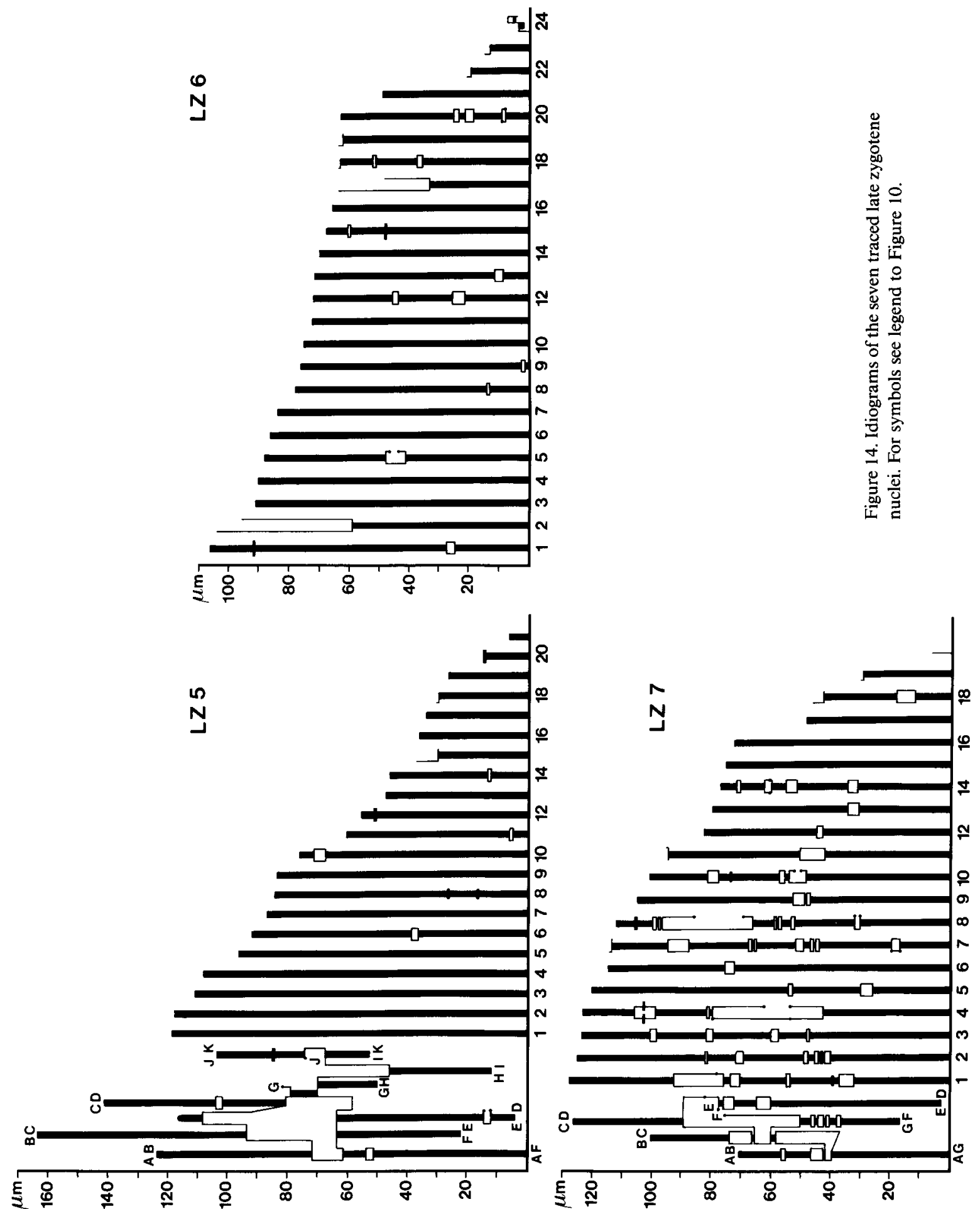
Table II.

Mean complement length (half of the lateral component length), degree of pairing in percent, number of synaptonemal complex (SC) segments and the number of chromosomes or chromosome fragments involved in multiple associations in the seven late zygotene nuclei.

s.d., standard deviation

\begin{tabular}{lllll}
\hline $\begin{array}{l}\text { Nucleus } \\
\text { number }\end{array}$ & $\begin{array}{l}\text { Complement } \\
\text { length }(\mu \mathrm{m})\end{array}$ & $\begin{array}{l}\text { Pairing } \\
\text { percent }\end{array}$ & $\begin{array}{l}\text { Number of } \\
\text { SC segments }\end{array}$ & $\begin{array}{c}\text { Number of chromosomes } \\
\text { in multiple } \\
\text { associations }\end{array}$ \\
\hline
\end{tabular}

\begin{tabular}{lrrrr} 
LZ1 & 1658 & 92.7 & 40 & 0 \\
LZ2 & 1641 & 92.9 & 39 & 0 \\
LZ3 & 1806 & 96.7 & 35 & 7 \\
LZ4 & 1911 & 93.3 & 41 & 7 \\
LZ5 & 1901 & 94.8 & 40 & 11 \\
LZ6 & 1664 & 94.2 & 38 & 0 \\
LZ7 & 2061 & 86.2 & 82 & 7 \\
\hline Mean & 1806 & 93.0 & 45 & \\
\pm s.d. & \pm 160 & \pm 3.3 & \pm 16 & \\
\hline
\end{tabular}

increase of the number of segments from 119 to 136 per nucleus between mid and late diplotene indicates that some interstitial degradation has occurred.

\section{DISCUSSION}

\subsection{The spreading procedure}

The present procedure developed for spreading wheat nuclei is a modification of the chromosome settling method employed by ALBINI, JONES and coworkers $(2,3)$ for spreading grasshopper spermatocytes and microsporocytes of Secale and Allium. In their procedure the anthers are macerated in a $1-2 \%$ solution of snail gut enzyme with $1 \%$ polyvinyl pyrrolidone and $1.5 \%$ sucrose. After a few minutes incubation, a detergent (Joy or Lipsol) is added to swell the nuclei. A similar procedure for plant microsporocytes has been developed by GILLIES (22) who macerated the anthers in Eagles medium containing 2 mM-EDTA and $0.1 \%$ bovine serum albumin and used the dishwashing agent Trix as the detergent.

The maceration and swelling media used in the present investigation were designed on the basis of the following experiments. Anthers containing microsporocytes at meiotic prophase were macerated in a variety of media with different concentrations of $\mathrm{Na}^{+}$and $\mathrm{Ca}^{2+}$, sorbitol, sucrose and with different buffers and at different $\mathrm{pH}$. The extruded nuclei were thereafter tested for their capability to swell by adding distilled water, while monitoring the process under phase contrast optics. The experiments revealed that intact nuclei could be obtained in media at a neutral $\mathrm{pH}$ containg $120-240 \mathrm{mM}$ $(0.5-1.0 \%) \mathrm{NaCl}$ or in $1 \mathrm{mM}^{-\mathrm{Ca}^{2+}}$ or in media with a slightly acidic $\mathrm{pH}$. It was not possible to obtain stable nuclei in media with a $\mathrm{NaCl}$ concentration at $60 \mathrm{~mm}$ or lower or in media with a neutral $\mathrm{pH}$ and a low $\mathrm{Na}^{+}$concentration since the nuclei started swelling and disintegrated immediately after their liberation from the pollen mother cells. Swelling of nuclei isolated in $120 \mathrm{~mm}-\mathrm{NaCl}$ was easily performed, whereas nuclei isolated in the presence of calcium or at low $\mathrm{pH}$ were difficult to swell and often contracted following the initial hypotonic shock. These results are in agreement with biochemical studies showing that chromatin is precipitated at a $\mathrm{Na}^{+}$concentration of 40-250 mM and at $\mathrm{a} \mathrm{Ca}^{2+}$ concentration of $1-10 \mathrm{~mm}$ (19). In the light of the present findings it is considered likely that the primary reason for the stability of the nuclei in the media employed by ALBINI, JONES and coworkers $(2,3)$ and GiLues $(22)$ is the low pH 

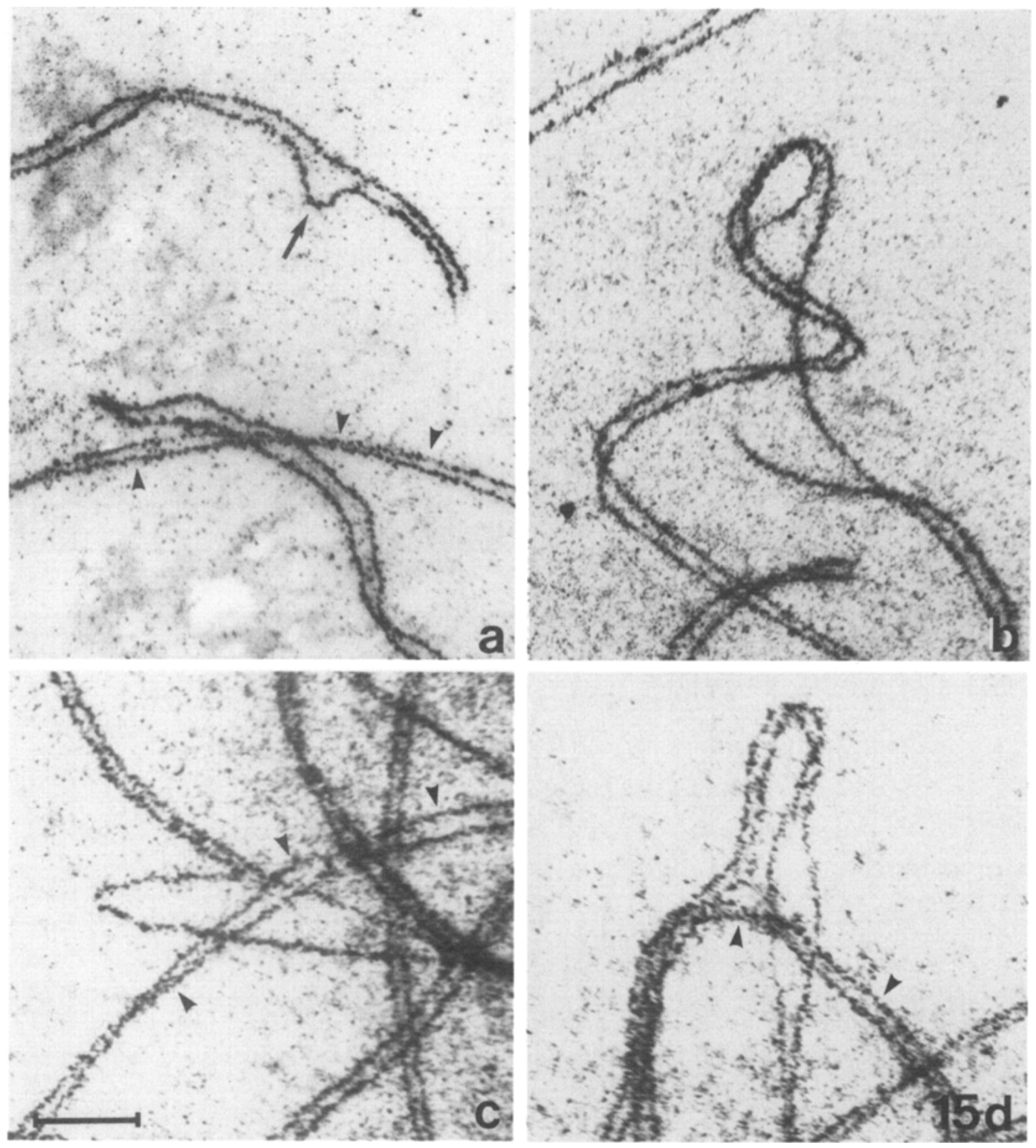

Figure 15. Examples of presumptive interlockings at late zygotene. In Figures 15a, $\mathrm{c}$ and $\mathrm{d}$ the bivalent marked by arrowheads appears to be interlocked between the lateral components of another bivalent. One of the interlocking lateral components in Figure $15 \mathrm{c}$ is somewhat longer than its partner (the involved chromosomes are chromosomes FG and AB in Figure 17b). The discontinuous lateral component in Figure 15b might reflect a resolution of an interlocking. Note the small lateral component loop in Figure 15a (arrow). (Bar $=1.0 \mu \mathrm{m}$ ).

of the former and the $\mathrm{Na}^{+}$concentration of Eagles medium in the latter procedure. Finally addition of EDTA appeared to facilitate the maceration of the anthers and the subsequent swelling of the nuclei.

A maceration medium was thus designed to contain a relatively low $\mathrm{NaCl}$ concentration $(0.5 \%)$, a high amount of chelating agent (10 

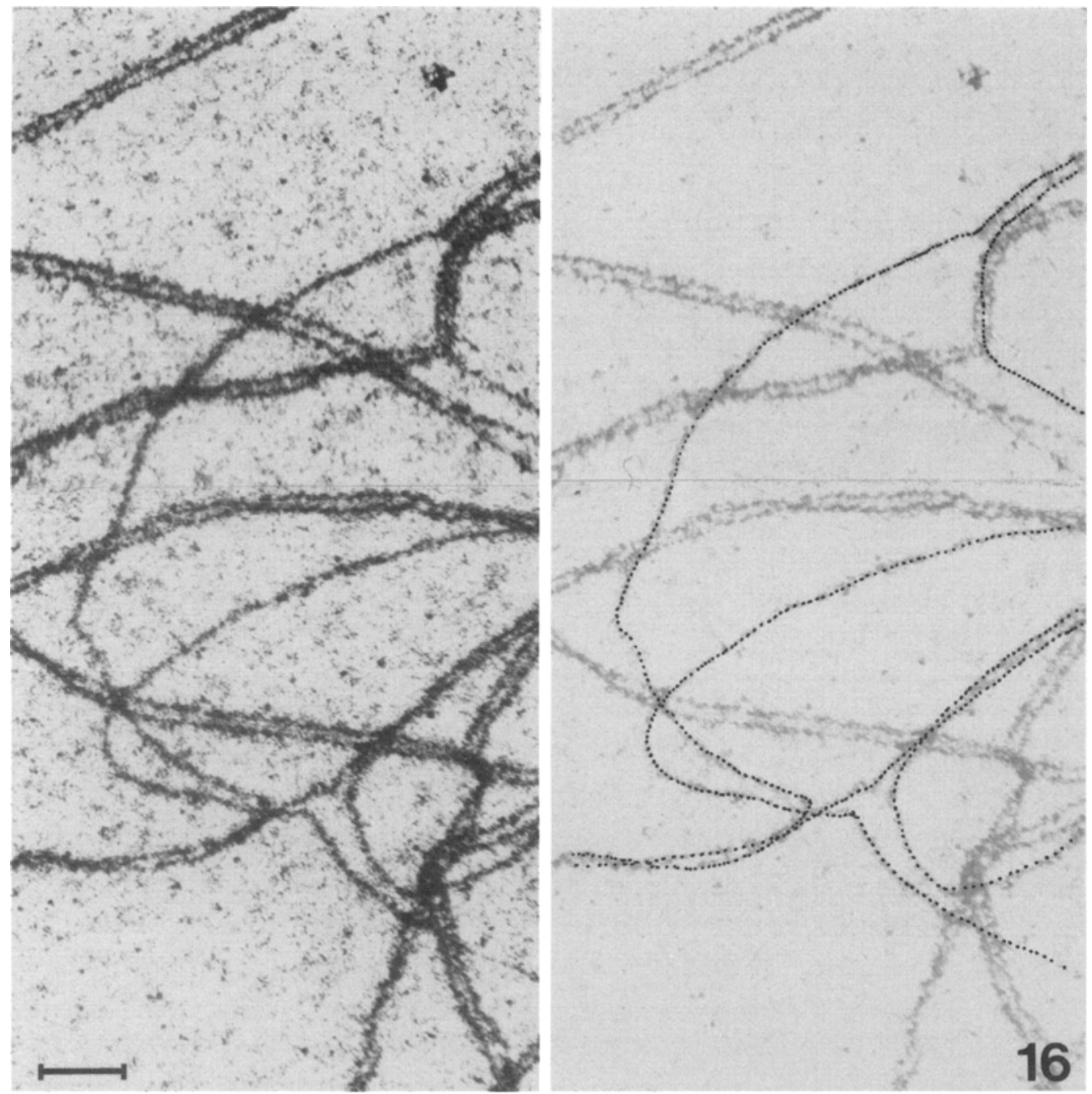

Figure 16. Micrographs and a tracing of a pairing partner exchange in a late zygotene nucleus (number LZ5). The entire multiple association is shown in Figure $17 \mathrm{c}$. (Bar $=1.0 \mu \mathrm{m})$.

mM-EDTA), some buffering capacity ( $6 \mathrm{mM}-\mathrm{Na}$ phosphate buffer) and a nearly neutral $\mathrm{pH}$. The presence of a weak ionic detergent in the swelling medium proved to be of importance to maintain the swollen state of the nuclei and perhaps also for the adherence of the nuclei to the plastic film. Strong detergents like sodium dodecyl sulphate (SDS) worked efficiently in the swelling process, but dissolved the nuclei during the long drying down period irrespective of the addition of formaldehyde. The dishwashing agent employed (Tex-I) contains the detergents sodium dodecyl benzene sulphonate and sodium dodecyl ether sulphate. The effect of these detergents separately and in combination on the spreading of meiotic nuclei of wheat are at present under investigation.

Breakage of lateral components and synaptonemal complexes is a major limitation in the use of spreading procedures for quantitative 
P B. Holm: Chromosome pairing in hexaploid wheat
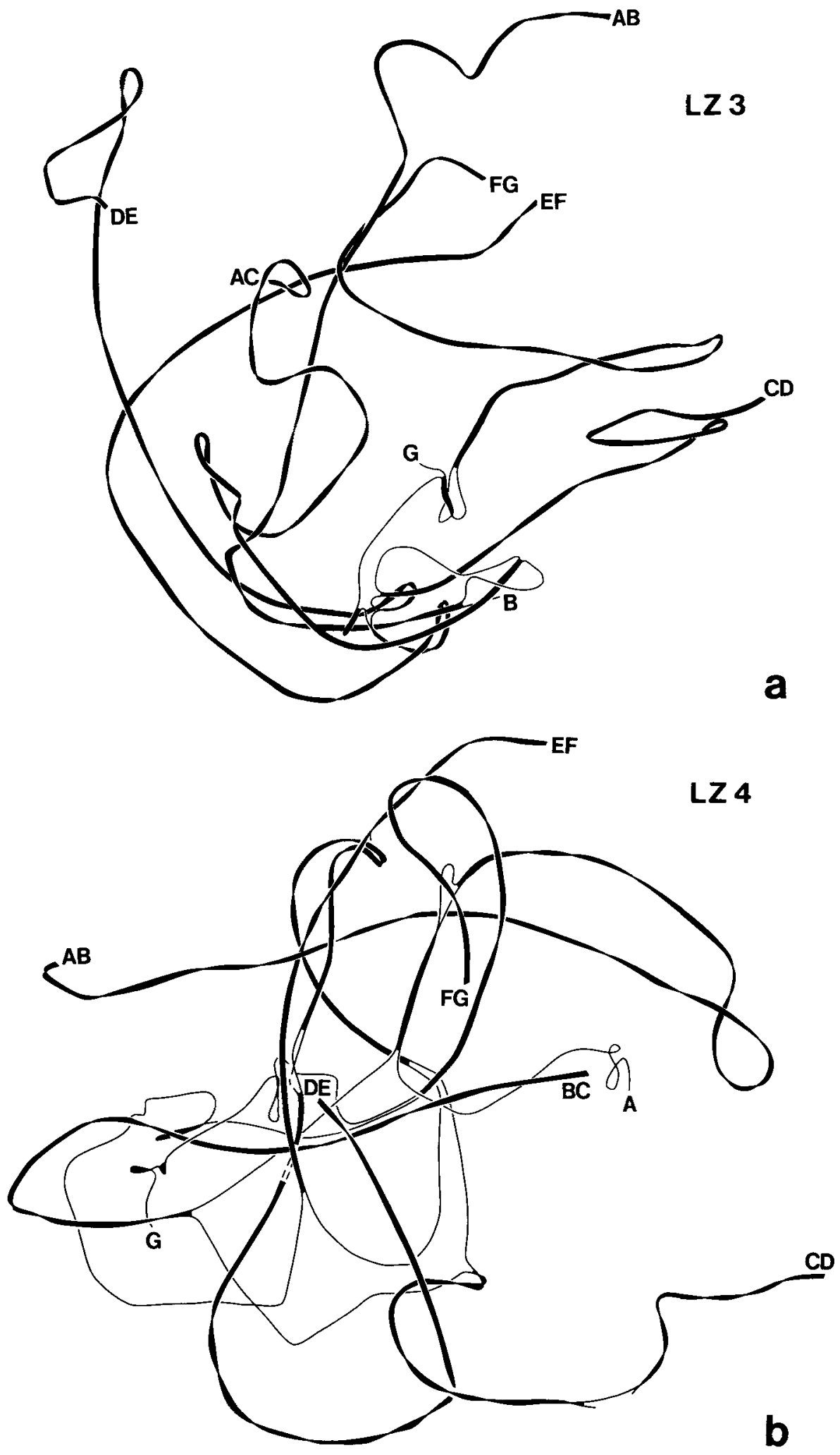

Carlsberg Res. Commun. Vol. 51, p. 239-294, 1986 

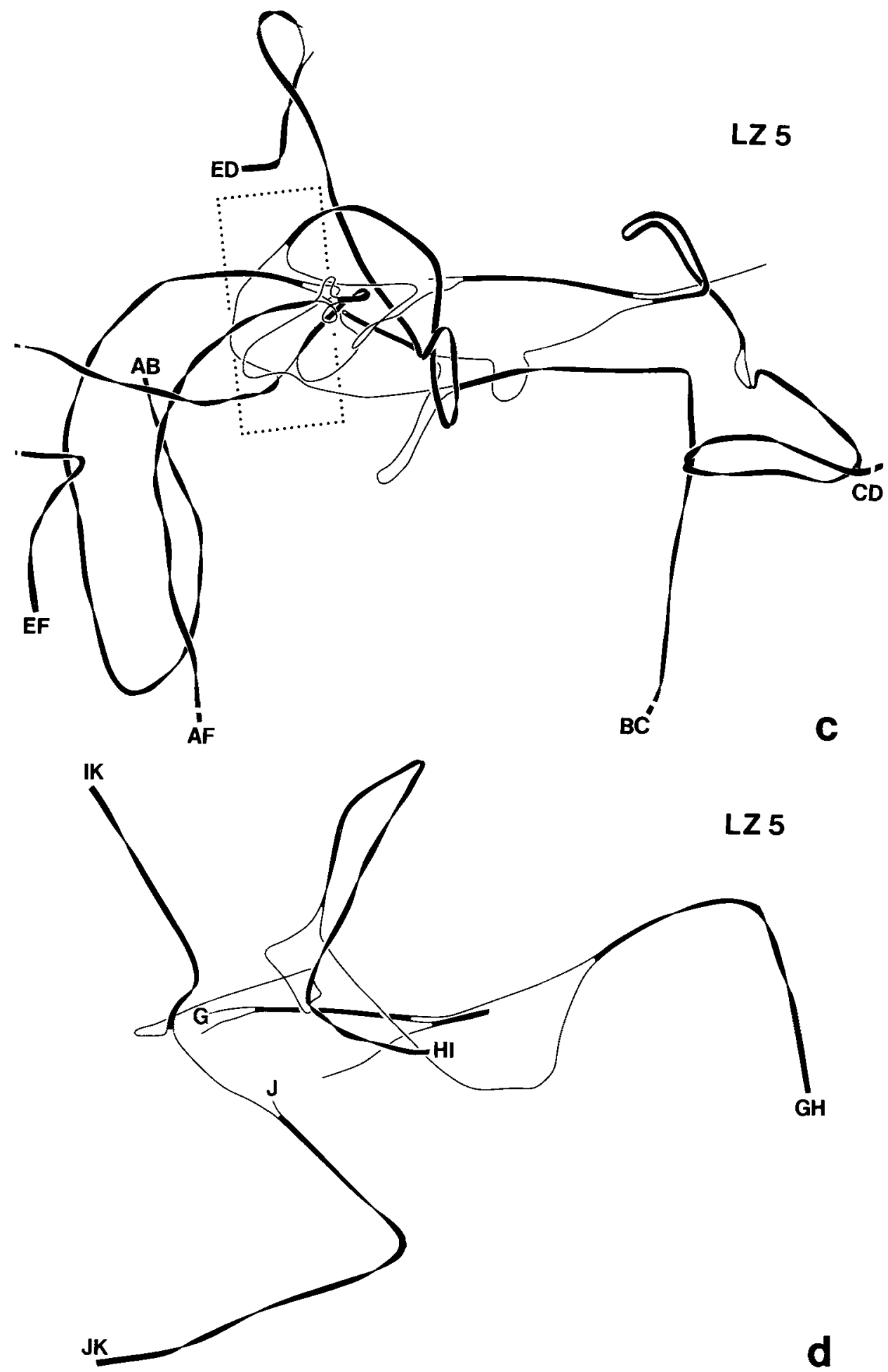


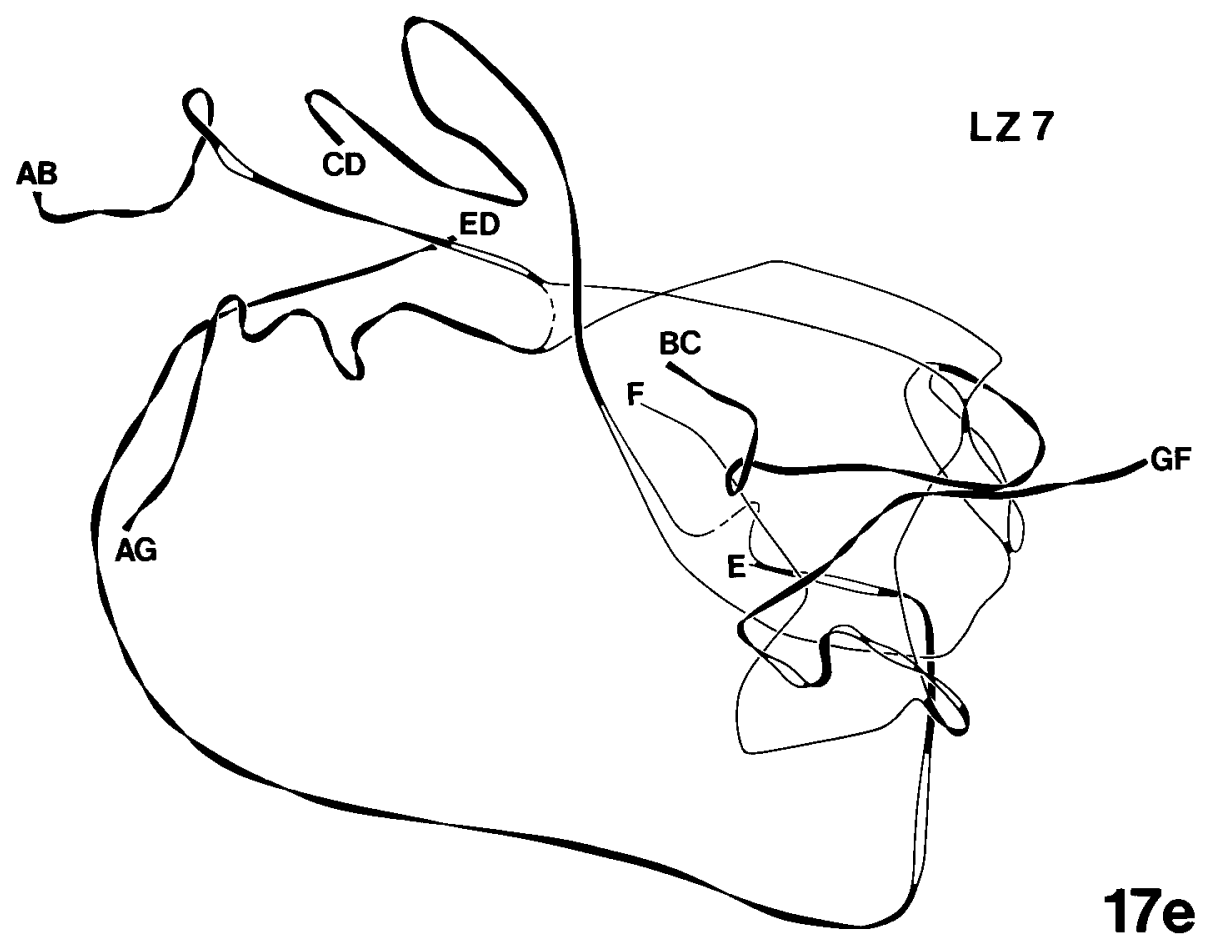

Figure 17. Tracings of the multivalents observed in the late zygotene nuclei. The numbers on the tracings refer to the number of the nucleus, and the letters denoting the individual chromosomes correspond to the letters used in the idiogram in Figure 14. A micrograph of the marked area in Figure 17c is shown in Figure 16. The hatched area in Figure 17d illustrates a part of a nucleolus. See also legend to Figure 4.

analysis of chromosome pairing in organisms with long chromosomes. Stretching, which eventually may lead to breakage of lateral components, appears to be an inherent problem in a procedure where nuclei are swollen and where the nuclear content collapses during air drying. Furthermore, the maceration of the anthers and the swelling of the nuclei probably liberates a variety of nucleases and proteases which may degrade the lateral components at least until formaldehyde is added. For these reasons the maceration, swelling and fixation was performed at $0-4{ }^{\circ} \mathrm{C}$, and high EDTA concentrations were used to inhibit nucleases. The brief post fixation with glutaraldehyde during washing was performed to stabilize the nuclei further before air drying and the inclusion of Ficoll in the washing medium has been reported to protect the nuclei during drying $(25,47)$. Altogether these precautions appeared to reduce the frequency of lateral component breakage.

\subsection{Chromosome pairing}

The present analysis of chromosome pairing in allohexaploid wheat has shown that with the exception of occasional exchanges of pairing partners to be discussed in section 4.5, chromosome pairing and synaptonemal complex formation proceeds as in diploid plants with similar chromosome lengths, i.e., Lilium (28), Secale (1, 22) and Tradescantia (25). A comparison with Secale is in particular relevant since the two genera are closely related and have partly homologous chromosomes (45). At the beginning of zygotene the telomeres aggregate and chromosome pairing and synaptonemal complex formation is preferentially initiated distally 
whereafter several interstitial segments synapse. Generally the longest synaptonemal complex segments seen at mid zygotene are those combining the distal segments in agreement with that pairing first is initiated at the telomeres. The number of synaptonemal complex segments per unit length of lateral component is similar in Triticum and Secale at the same stage of zygotene. ABIRACHED-DARMANCEY et al. (1) thus observed 96 synaptonemal complex segments in a $65 \%$ paired zygotene nucleus, while Gillies (22) found from 48-76 (mean of 59) segments per nucleus in six nuclei with a mean complement length of $610 \mu \mathrm{m}$, ranging in pairing from 40 to $77 \%$. The number of synaptonemal complex segments per $100 \mu \mathrm{m}$ of complement is thus 7.4 in Triticum and 9.7 in Secale at the same stage of pairing. In Lilium, 170 segments were observed in a nucleus $22.1 \%$ paired and a complement length of $3695 \mu \mathrm{m}$. HASENKAMPF (25) estimated the "potential number" of initiation sites for synaptonemal complex formation in six nuclei of Tradescantia ranging in synapsis from 3.5 to $64.4 \%$ and in the reconstructed zygotene nucleus of Lilium. This estimate was obtained by dividing the length of unpaired segments exceeding $50 \mu \mathrm{m}$ in length by the mean distance between mid points of adjacent synaptonemal complex segments. This number was then added to the number of observed initiation sites. It was estimated that in Lilium there were 12.4 initiation sites per 100 $\mu \mathrm{m}$ of complement, while in a Tradescantia nucleus with $24 \%$ pairing and a complement length of $2537 \mu \mathrm{m}$ the number of initiation sites was 9.9 per $100 \mu \mathrm{m}$. These figures are similar to those of Triticum and Secale and it appears that the kinetics of chromosome pairing and synaptonemal complex formation is similar in these four plants.

Based on observation from Lilium it was proposed (28) that synaptonemal complex formation could be initiated anywhere along the lateral components as evidenced by: I) Synaptonemal complex formation can be initiated in all segments, distal, interstitial and proximal. II) New segments of synaptonemal complex can form very close to other segments. III) There is no preferential distance between the neighbour segments. A similar conclusion can be drawn from the investigations of Secale, Tradescantia and Triticum. In these three organisms synapsis is not confined to a particular chromosome segment, new segments of synaptonemal complex may form at a center to center distance to adjacent ones as low as $0.5 \mu \mathrm{m}$ (this study) or 0.6 $\mu \mathrm{m}$ (Secale, 22), and in the frequency distribution histograms of distances between adjacent segments presented for Tradescantia and Secale there is no indication of periodicity. A comparison of the distribution of paired segments along the individual bivalents of Secale in three zygotene nuclei with 60,83 and $95 \%$ of their complement paired did likewise not suggest a bivalent specific pattern in the initiation and progression of synaptonemal complex formation (22).

In Lilium measurements of the length of both lateral components of the unpaired regions revealed that they generally were of the same length, even in segments more than $100 \mu \mathrm{m}$ long. This shows that the length of homologous lateral components is determined before they are paired with a synaptonemal complex. This appears also to be the case in Secale and Tradescantia and is documented in the present study where measurements of lateral component length in unpaired segments at mid zygotene have shown an equality in length of the lateral components in $93 \%$ of the segments in which both lateral components are continuous. In the remaining seven percent of the segments the lateral components'have different lengths, most of these differences can be attributed to interlocking as described in sections 3.2.1 and 3.2.2.

These observations are in agreement with the general conclusion that the zygotene pairing is highly specific (70) and further indicate that the information for a specific pairing may be housed all along the chromosome $(28,69)$. There are, however, a few examples of nonhomologous pairing during zygotene. In the present material as well as in Secale (22), Tradescantia (25), Homo $(9,52)$, Bombyx (30) and Lilium (28) fold-back pairing of unpaired lateral components is occasionally seen, while exchanges of pairing partner are very rare in the disploid species. In several cases these illegitimate pairing configurations can be attributed to interlockings, whereby nonhomologous chromosomes 


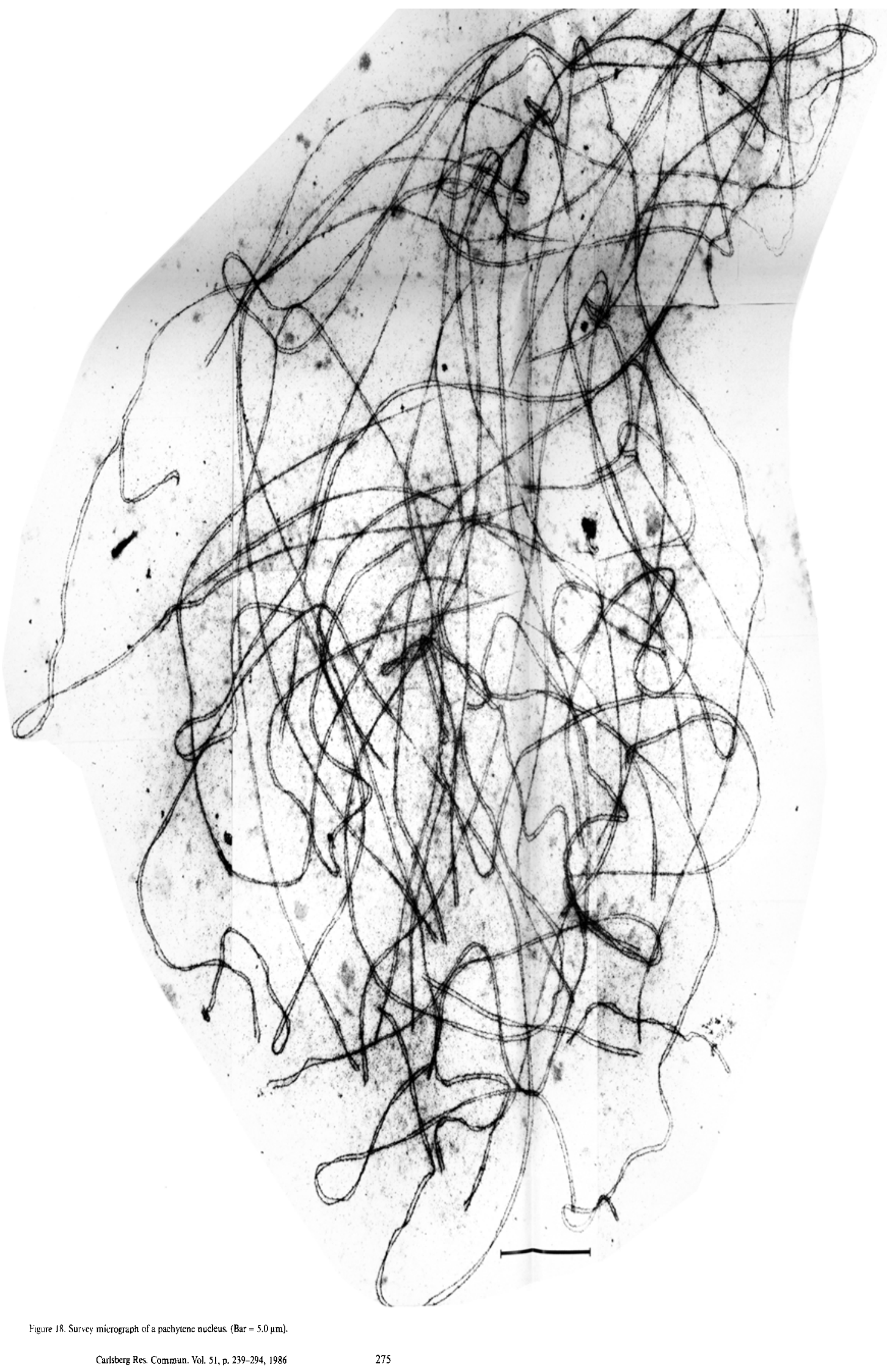


Table III.

Total complement length (half the total lateral component length) and degree of pairing in twenty pachytene nuclei.

s.d., standard deviation

\begin{tabular}{lcc}
\hline $\begin{array}{l}\text { Nucleus } \\
\text { number }\end{array}$ & $\begin{array}{c}\text { Complement } \\
\text { length }(\mu \mathrm{m})\end{array}$ & $\begin{array}{l}\text { Pairing } \\
\text { percent }\end{array}$ \\
\hline P1 & 1693 & 97.3 \\
P2 & 1348 & 99.0 \\
P3 & 1382 & 99.1 \\
P4 & $1884^{\mathrm{a}}$ & $64.6^{\mathrm{a}}$ \\
P5 & 1972 & 96.9 \\
P6 & 1609 & 90.4 \\
P7 & 1487 & 95.5 \\
P8 & 1285 & 86.9 \\
P9 & 1885 & 92.7 \\
P10 & 1412 & 99.3 \\
P11 & 1264 & 97.7 \\
P12 & 1525 & 99.4 \\
P13 & 1194 & 99.2 \\
P14 & 1346 & 99.8 \\
P15 & 1424 & 96.7 \\
P16 & 1258 & 94.9 \\
P17 & 1448 & 98.5 \\
P18 & 1258 & 96.5 \\
P19 & 1620 & 99.4 \\
P20 & 1613 & 99.0 \\
\hline Mean & 1474 & 96.7 \\
\pm s.d. & \pm 214 & \pm .5 \\
a) not included in the mean & \\
\hline
\end{tabular}

may be brought into proximity for a sufficient time to trigger synaptonemal complex formation $(30,52)$. The "skewed" pairing observed in the present study and in Secale (22) may represent an additional example of nonhomologous pairing at zygotene. As described in section 3.2.3, this pairing configuration appears to result from interlocking whereby the alignment process and subsequent synapsis of a segment within this region is disturbed.

Impediment of chromosome pairing by interlocking and stretching of lateral components may in particular be of importance towards the end of zygotene and at pachytene. It is thus well established that pairing and synaptonemal complex formation between nonhomologous segments occurs much more readily at pachytene than at zygotene (70). Interlocking and stretching of lateral components may thus account for the occasional misalignment of centromeres in synaptonemal complex segments of wheat (27, 38) and triploid Allium sphaerocephalum (43) and may also be the mechanism responsible for the presence of small unpaired loops of one of the lateral components in otherwise completely synapsed bivalents of diploid Lolium (39). Small loops have occasionally been seen in late zygotene and pachytene nuclei of wheat in this

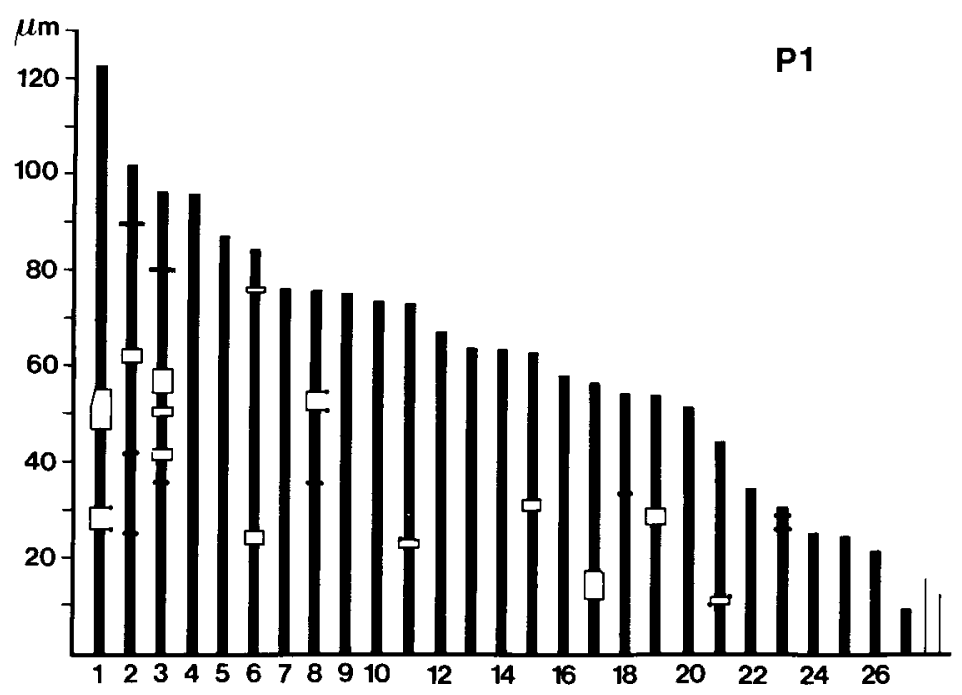

Figure 19. An idiogram of a pachytene nucleus. See also legend to Figure 10. 

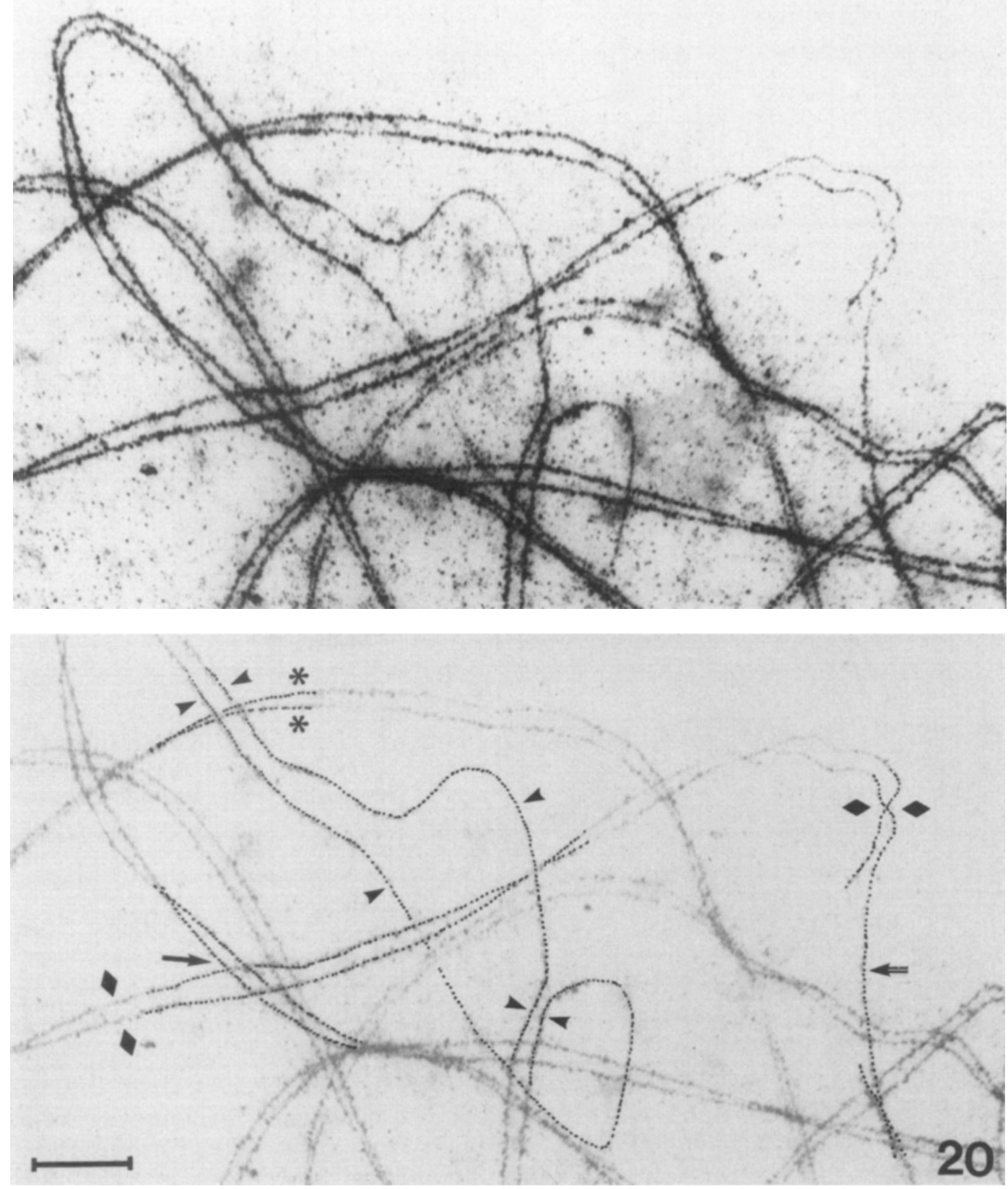

Figure 20. A micrograph and a tracing of presumptive bivalent interlockings in a pachytene nucleus. The bivalent marked by arrowheads appears to interlock two other bivalents marked by asterisks and diamond symbols. The bivalent denoted by diamond symbols appears to interlock another bivalent (arrow) and one of its lateral components is discontinuous on the right side in the micrograph (double arrow). (Bar $=1.0 \mu \mathrm{m}$ ).

analysis (Figure 15a) and in the late zygotene nucleus of wheat reconstructed by HOBOLTH (27).
The incomplete synapsis in several of the wheat microsporocytes remains at present unexplained. Wheat lines nullisomic for chromo- 


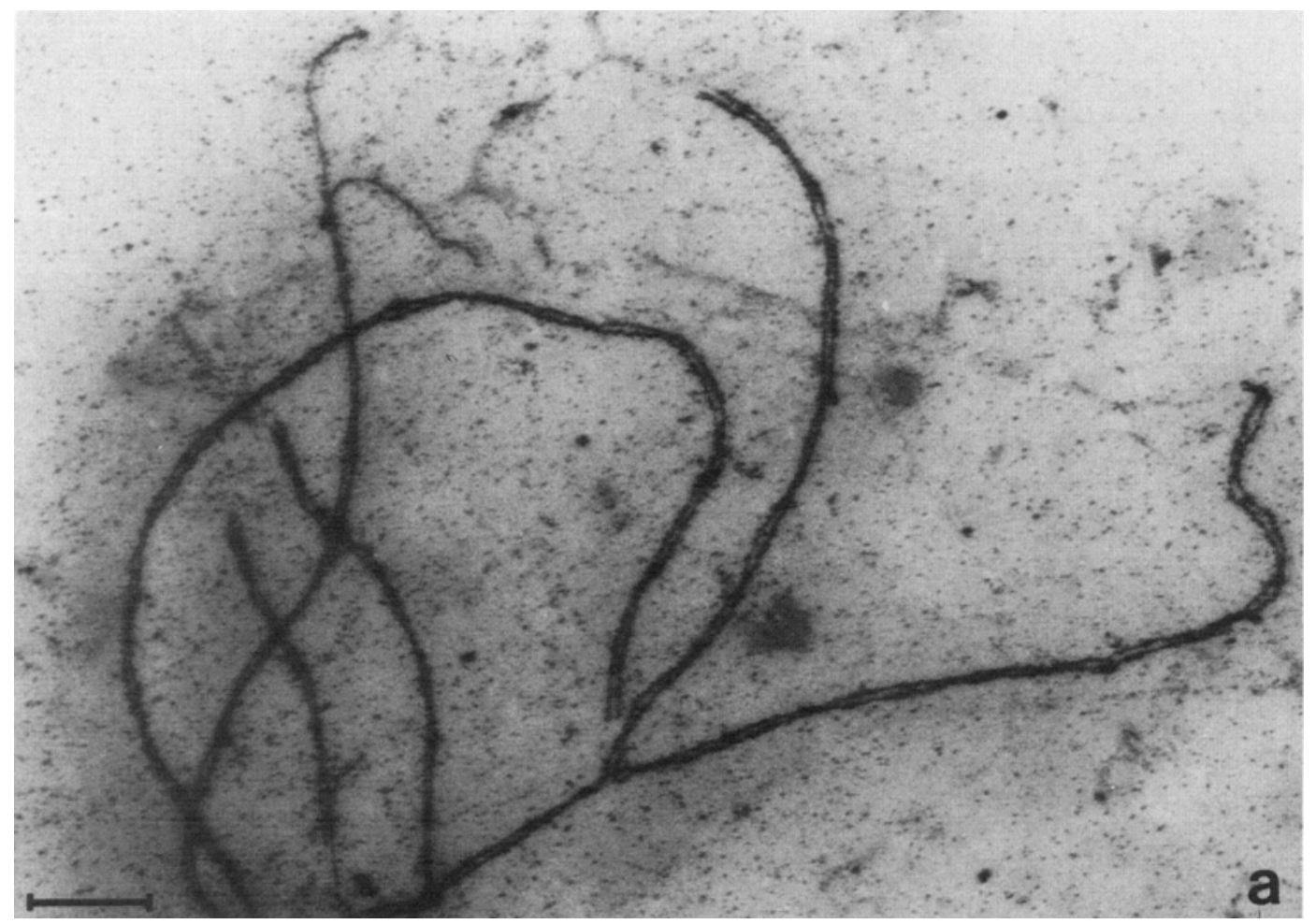

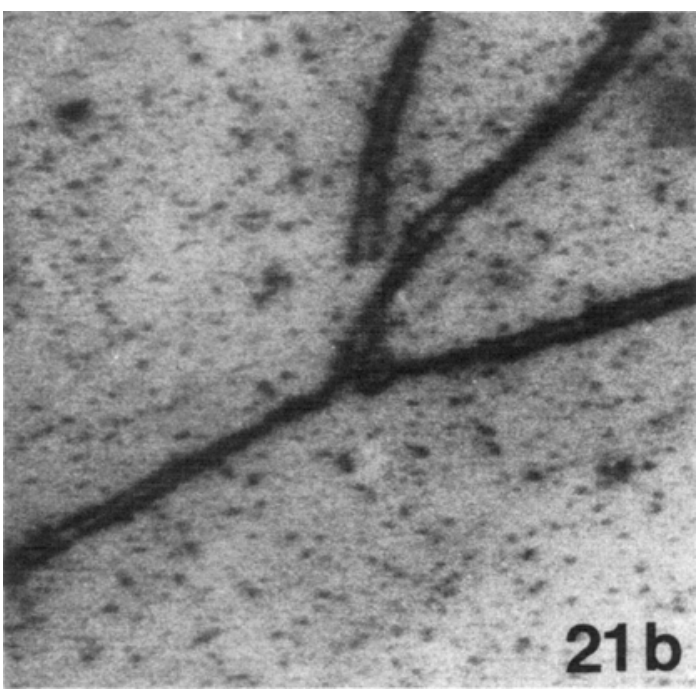

Figure 21. Micrographs of a pairing partner exchange (trivalent) in a pachytene nucleus. A higher magnification of the exchange region is shown in Figure $21 \mathrm{~b}$. (Bar $=2.0 \mu \mathrm{m}$ ). some 5D or diisosomic for the long arm of chromosome 5B have been reported to be completely or partly achiasmatic if grown at $15^{\circ} \mathrm{C}$, an effect which may be attributed to asynapsis $(7,56)$. Synapsis in normal wheat may also be affected by changing the environmental conditions. This possibility is at present under investigation. It might also be of interest to analyze whether the incomplete synapsis is a characteristic of the cultivar Chinese Spring and of possible 
significance for the frequent formation of aneuploids.

\subsection{Complement length}

The mean complement length at mid zygotene, late zygotene and pachytene amounted to $2063 \mu \mathrm{m}, 1806 \mu \mathrm{m}$ and $1474 \mu \mathrm{m}$. There is thus a gradual reduction in length of the lateral components, the pachytene length only being about two-thirds of the length observed at mid zygotene. The mid zygotene lengths are very similar to those of early zygotene in plants triisosomic for the long arm of chromosome 5B (71), where the mean complement length of five spread nuclei with $25 \%$ pairing was $2073 \mu \mathrm{m}$, while a reconstructed nucleus with $25 \%$ pairing had a complement length of $1722 \mu \mathrm{m}$. The shortening of the complement from late zygotene to pachytene is also apparent from the data presented by НовОLTH, where the complement length was $1897 \mu \mathrm{m}$ at late zygotene and $1235 \mu \mathrm{m}$ at pachytene (27). A similar reduction in length from the beginning of zygotene to pachytene has been reported for rye where the complement length decreased from a mean of 6-700 $\mu \mathrm{m}$ at mid zygotene to a mean of $437 \mu \mathrm{m}$ at pachytene (22). The wheat complement is thus roughly three times longer than the rye complement in agreement with the three fold difference in number of similar sized chromosomes. A reduction in length from zygotene to pachytene is also reported for maize (21) but is not a general feature of meiosis since the zygotene and the pachytene complements are roughly of the same length in a number of fungi, insects and mammals (70).

\subsection{Interlocking}

Interlockings are abundant in the analyzed mid zygotene nuclei. The estimated interlocking frequency of about 20 per nucleus is thus a minimum estimate at this stage. A substantial number of the unpaired segments of the complement are involved in interlocking events which probably impede the progression of chromosome pairing and synaptonemal complex formation. Most of the interlockings are bivalent interlockings in which a bivalent segment is trapped between a pair of unpaired homologues. As pairing progresses most of the interlockings are resolved by breakage and reunion of one or more of the involved chromosomes and bivalents, but even at a stage where $95 \%$ of the complement is synapsed, there is a least a mean number of five bivalent interlockings per nucleus. In the pachytene nuclei with almost complete pairing nearly all interlockings have disappeared. In nuclei with an incomplete synapsis some of the interlockings may persist into diplotene.

The observed interlocking frequency is much higher than that reported for the two serially sectioned and reconstructed early and late zygotene nuclei of wheat where only a single interlocking event was found in each nucleus $(27,38)$. The reasons for this discrepancy is obvious. The reconstructed late zygotene nucleus was nearly completely paired and virtually all interlockings had accordingly been eliminated, while only partial reconstructions were possible in the reconstructions of the early zygotene nucleus due to the presence of knots of entangled lateral components and synaptonemal complexes which generally were difficult to trace through the knots. As proposed by JENKINS (38) these knots may represent regions of multiple interlockings.

Interlockings can be maintained up to metaphase $I$ in wheat microsporocytes. YACOBI et al. (72) thus reported that $4 \%$ of the metaphases contained an interlocking. The validity of this estimate has, however, recently been questioned by HESLOP-HARRISON and BENNETT (26), who observed one chromosome interlocking and one bivalent interlocking in six serially sectioned and reconstructed meiotic metaphases of wheat. In two of these nuclei closely appressed bivalents were observed possibly indicating interlocking of these bivalents. It was argued that since it is often difficult to score for interlockings at metaphase I in wheat using light microscopic techniques, the frequency of interlocking at metaphase I may be somewhat higher than $4 \%$.

It is likely that retention of interlocking up to metaphase $I$ is related to the fact that transition from zygotene to diplotene in wheat is not dependent on the completion of synaptonemal 
complex formation. Interlockings appear to be resolved if the lateral components in a region of interlocking are brought into contact either due to progression of synaptonemal complex formation between the interlocking lateral components. or if the interlocked and interlocking chromosomes move in different directions. When synaptonemal complex formation ceases prematurely as the nuclei enter the pachytene stage, interlockings may no longer be recognized and resolved. Hence, the frequency of interlocking at metaphase I may be taken as a quantitative measure of the amount of complete pairing. Y ACOBI et al. (72) thus noted a linear correlation between the number of interlockings per ring bivalent at metaphase $I$ and the number of copies of the long arm of chromosome $5 \mathrm{~B}$ within the range from two to six copies. The freqency ranged from 0.002 in euploid wheat to 0.135 in plants triisosomic for the long arm of chromosome $5 \mathrm{~B}$. This effect was attributed as a dosis effect of the $\mathrm{Ph}$ gene on a premeiotic arrangement of the chromosomes (see section 4.7). As discussed by WISCHMANN (71) an increase in copy number of the iong arm of chromosome $5 \mathrm{~B}$ from two to four leads to pairing arrest at mid zygotene and six copies causes the pairing to stop at early zygotene. This shows that there is a correlation between the degree of synapsis and the interlocking frequency at metaphase $I$.

The frequency of interlocking at zygotene in a number of organisms ranging from fungi to man indicates that the number of interlocking events relates to the number and length of the chromosomes (33). Quadrivalent formation due to heterozygosity for a reciprocal translocation also greatly increases the frequency of interlocking $(33,34)$. The extensive zygotene interlocking reported for the long chromosomes of Secale supports this conclusion. ABIRACHED-DARMEN$C Y$ et al. (1) thus observed four bivalent and three chromosome interlockings in a reconstrueted zygotene nucleus which was $65 \%$ paired while GILLIES (22) found 9 interlockings pius 6 presumptive interiockings in a spread nucleus with $60 \%$ pairing and 9 interlockings and 2 presumptive interlockings in a nucleus where $83 \%$ of the complement had synapsed. In wheat the number of interlockings appears to be somewhat higher than in rye in agreement with the tinree- fold larger chromosome number and complement length and the occurrence of multiple associations.

\subsection{Chiasma formation}

The orderly degradation of the synaptonemal complex during diplotene is clearly revealed in the spread nuclei. It is apparent that the degradation is initiated at a low number of sites at early diplotene, generating on the average $89 \mathrm{seg}$ ments. During mid and late diplotene degradation is primarily from the ends of those segments and less frequently interstitially as evidenced by the rather modest increase in the number of segments at mid and late diplotene (119 and 136 segments). This degradation process is essentially similar to that reported for human spermatocytes (32). During diplotene the number of distinct synaptonemal complex segments remained almost constant with a mean of 72 segments per nucleus. In addition a mean number of 23 short and partly degraded segments was found at mid and late diplotene, generally at the ends of the distinct segments. Such a dimorphism is noc seen in wheat, where virtually all segments at early and mid dipiotene appeared to possess a normal morphology. The extensive thickening of the lateral components in some synaptonemal complex segments has so far not been described for other organisms with the exception of the achiasmatic diploid oocytes of the silkworm (51). Hiere the entire synaptonemal complex is retained between the homologues up to metaphase I to compensate for the lack of crossing over. During diplotene the lateral components in Bombyx oocytes enlarge in diameter and enguli eventually the centra. region. The significance of a similar modification of the laieral componen is in wheat is at present unclear. WischMANN (71) reported from a study of wheat triisosomic for the long arm of chromosome $5 \mathrm{~B}$ that in most late diplotene nuclei two distinctively different types of synaptonemal complex segments were retained, one with thickened lateral components and one with lateral components of normal appearance. A similar dimorphism is noc seen in normal wheat. where a cont:nuum of lateral component wioths is revealed 
As initially proposed from observations in Neottiella $(67,68)$ and the mouse (62) and later confirmed in a number of other organisms (70), synaptonemal complex segments are retained during diplotene where crossing over has taken place. It has thus been possible to estimate the number of crossovers in e.g., the human male and female by counting the number of retained synaptonemal complex segments at diplotene $(10,32)$. In both cases the number of segments correlated well with the number of crossovers inferred from the study of recombination nodules. This appears also to be the case e.g., in Sordaria (73) and Neurospora (20), where nearly all synaptonemal complex segments retained at diplotene possessed a recombination nodule.

The total length of the genetic map in wheat is not known. Around 40 chiasmata are consistently reported at metaphase I, most of them being terminal. Although there is some evidence for a distal localization of crossovers in wheat (16), terminalization may have reduced the number of recognizable chiasmata considerably up to metaphase I. The number of crossovers may therefore be considerably larger than 40 per nucleus.

HовоLTH (27) observed 88 recombination nodules in a reconstructed pachytene nucleus of wheat. The identity between this number and the mean number of synaptonemal complex segments at early diplotene ( 89 segments per nucleus) may indicate that the frequency of crossing over is of this order of magnitude. Several of the segments at mid and late diplotene may thus represent partially degraded segments devoid of crossovers.

\subsection{Multiple associations and their correction}

At mid zygotene eight of the eleven completely traced nuclei contain a total of eleven multiple associations. Four of the seven late zygotene nuclei contained a total of five multiple associations, and at pachytene only five associations were found in twenty nuclei. With one possible exception multiple associations were not identified at diplotene.

It can thus be concluded that maltiple association of chromosomes regularly form during the zygotene chromosome pairing in allohexapioid wheat and that these multiple associations subsequently are corrected into bivalents. Multiple associations, consisting of two bivalents held together by short interstitial segments of central region (type II) are first eliminated as evidenced by the scarcity of this type at late zygotene and pachytene. In contrast, multiple associations of type I which are characterized by a single exchange of pairing partner per chromosome are maintained through late zygotene and in most cases corrected during pachytene. As described for autotetraploid silkworm oocytes (53) the correction of such associations probably involve a gradual migration of the site of pairing partner exchange to the telomeres by a dissolution of the central region material in front of this region and reformation of the central region between the alternative combination of lateral components behind this region.

The results of this study are in agreement with those presented previously from serially sectioned and three dimensionally reconstructed zygotene nuclei of wheat. JENKINS (38) thus observed a type I multiple association of five lateral component segments at early zygotene whereas HOBOLTH (27) in a late zygotene nucleus found three single exchanges of lateral components (Type I) and eight double exchanges (Type II). The latter type of association differed, however, slightly from those seen in the present study since all four lateral components exchanged pairing partner over a short distance. Results similar to those obtained in the present study have been obtained by GILLIES (manuscript in preparation), who at the zygotene-pachytene transition in allohexaploid wheat observed up to two multiple associations per nucleus.

Recent studies have supported the contention by HовоLTH (27) that multivalent formation at zygotene and the subsequent transformation of multivalents into bivalents is a regular feature of meiosis in allopolyploids in which only bivalents are present at metaphase I. Correction of chromosome pairing in an allotriploid hybrid $(3 x=21)$ of Lolium perenne and Lolium temulentum containing two genomes of L. perenne, one genome of L. temulentum and two B-chromosomes has recently been reported by JENKINS (40). A reconstructed early zygotene nucleus contained six bivalents of the L. perenne chro- 


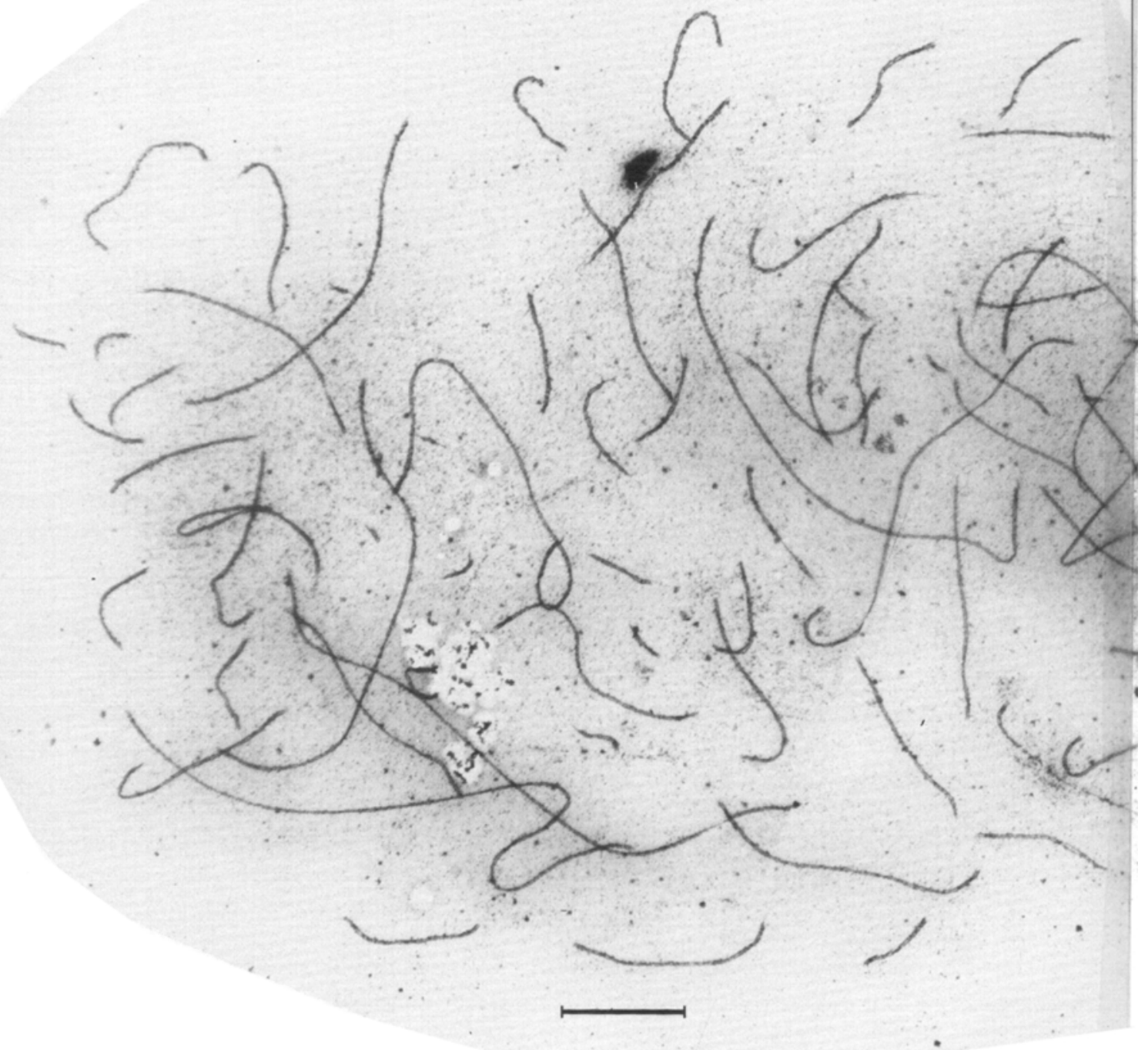

Figure 22. A survey micrograph of an early diplotene nucleus of wheat. The letter $\mathbf{N}$ marks the faintly stained nucleolus. $(\mathrm{Bar}=5.0 \mu \mathrm{m})$. 


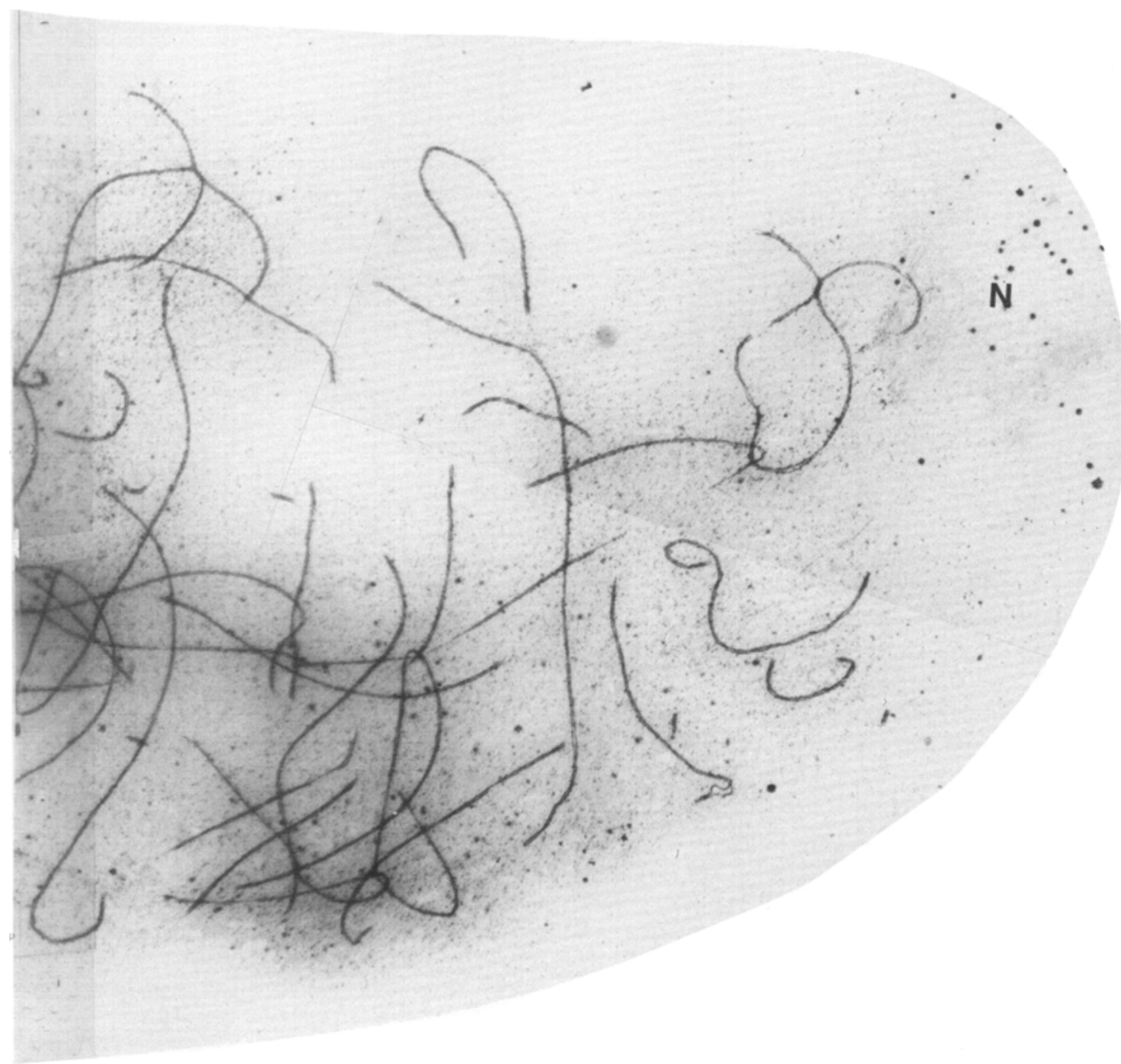


Table IV.

The number and length of the synaptonemal complex (SC) segments and haif the Sength of unpaired lateral components (LC) in 12 early diplotene nucle.

s.d., standard deviation

\begin{tabular}{|c|c|c|c|}
\hline $\begin{array}{l}\text { Nucleus } \\
\text { number }\end{array}$ & $\begin{array}{l}\text { Number of } \\
\text { segments }\end{array}$ & $\begin{array}{l}\text { SC length } \\
(\mu \mathrm{m})\end{array}$ & $\begin{array}{l}1 / 2 \text { unpaired } \\
\text { LC length } \\
(\mu \mathrm{m})\end{array}$ \\
\hline ED 1 & 106 & 695 & 273 \\
\hline ED 2 & 118 & 1280 & 218 \\
\hline ED 3 & 76 & 815 & 6 \\
\hline ED 4 & 96 & 733 & 265 \\
\hline ED 5 & 69 & 1213 & 118 \\
\hline ED 6 & 113 & 1077 & 161 \\
\hline ED 7 & 77 & 1118 & 68 \\
\hline ED 8 & 97 & 679 & 475 \\
\hline $\mathrm{ED} 9$ & 44 & 812 & 337 \\
\hline ED 10 & 89 & 919 & 27 \\
\hline ED 11 & 97 & 1088 & 0 \\
\hline ED 12 & 80 & 918 & 0 \\
\hline Mean & 89 & 946 & 162 \\
\hline \pm s.d. & \pm 21 & \pm 206 & \pm 154 \\
\hline
\end{tabular}

mosomes, a B-chromosome bivalent, six univalents (L. temulentum chromosomes) and a trivalent consisting of two perenne and one temulentum chromosome. In contrast a reconstructed pachytene nucleus contained seven bivalents, seven univalents with extensive nonhomologous pairing and a B-chromosome bivalent. These results are similar to those of wheat showing that the initial pairing preferentially is between homologues, and that multivalents which occasionally have formed are prone to correction. Reconstructions of an early zygotene nucleus of an allotetraploid hybrid ( $2 \mathrm{n}=4 \mathrm{x}=28$ ) of Lolium with four B-chromosomes revealed seven bivalents, one trivalent, one quadrivalent, one pentavalent and one fold-back paired univalent while two nuclei, which were considered to be at late pachytene each contained 10 bivalents, a quadrivalent of B-chromosomes, some multivalents and fold-back paired univalents. At metaphase only bivalents and univalents were observed in the light microscope. The increase in the number of bivalents from zygotene to pachytene indicates that a few multivalents have been transformed (41).
As described in the introduction, evidence from a number of organisms suggest that transformation of multiple associations into bivalents is prevented by the occurrence of crossing over in the paired segments flanking the region of pairing partner exchange. Ultrastructural studies of a number of organisms such as Drosophila (11), Bombyx (30), Coprinus (34), Homo (10, 31, 52) and mouse (23) (see 29, 70 for review) have thus indicated that crossing over first occurs at mid and late pachytene and is marked by structural, numerical and distributional changes of recombination nodules (29). Thus, at early pachytene the recombination nodules transform from small, generally spherical structures to larger electron dense and often elongated types. This appears also to be the case in rye (1), and large dense nodules are apparent at pachytene in wheat (27). Preliminary observations of spread pachytene and diplotene nuclei stained with phosphotungstic acid have revealed nodules of this large type.

It is apparent from a number of investigations that correction of chromosome pairing extends into mid pachytene (70), i.e., concurrently with 


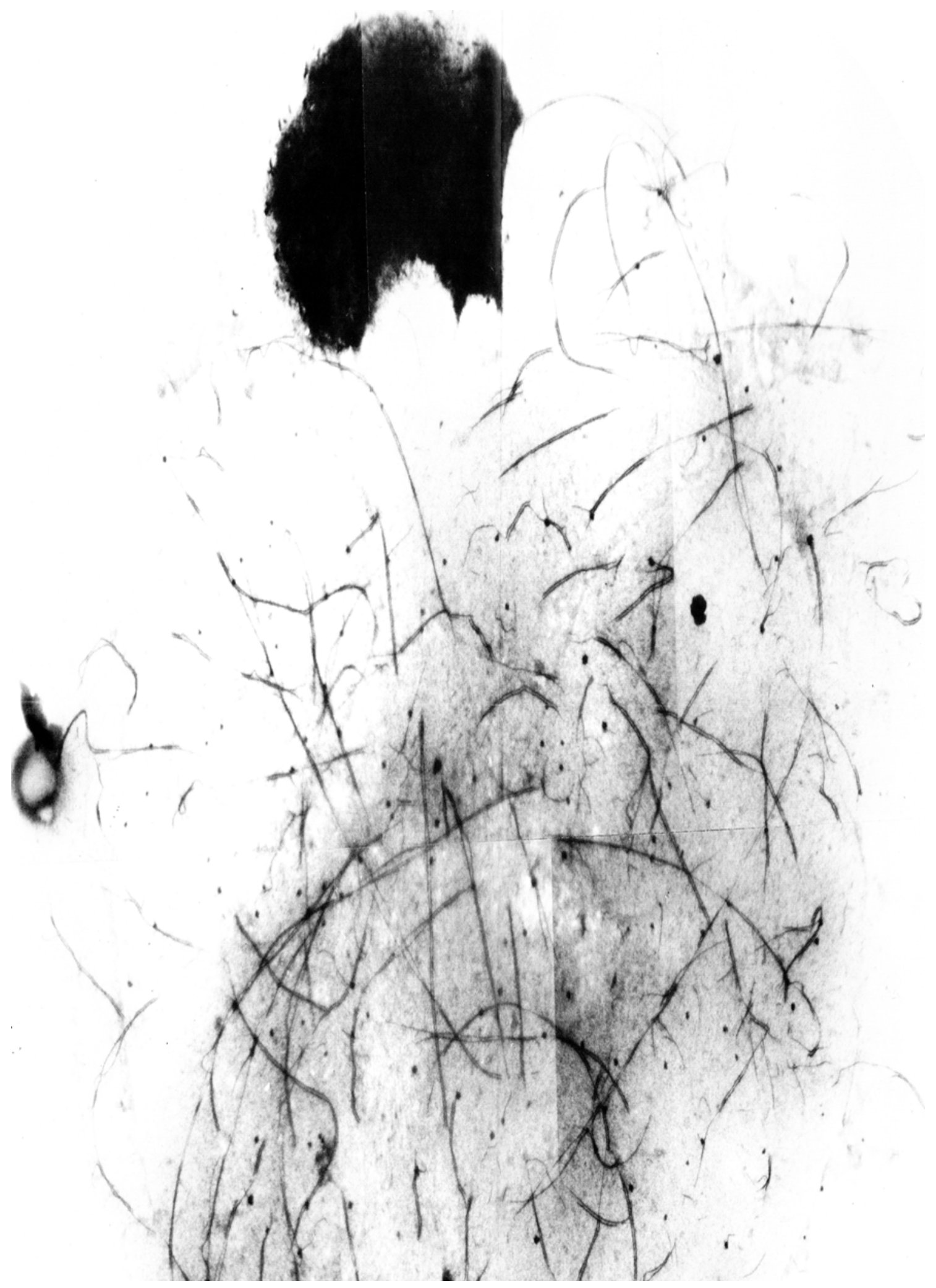

Figurc 23. A survey micrograph of an early diplotene nucleus in which pairing has not been completed. (Bar = $=5.0$

uml. 


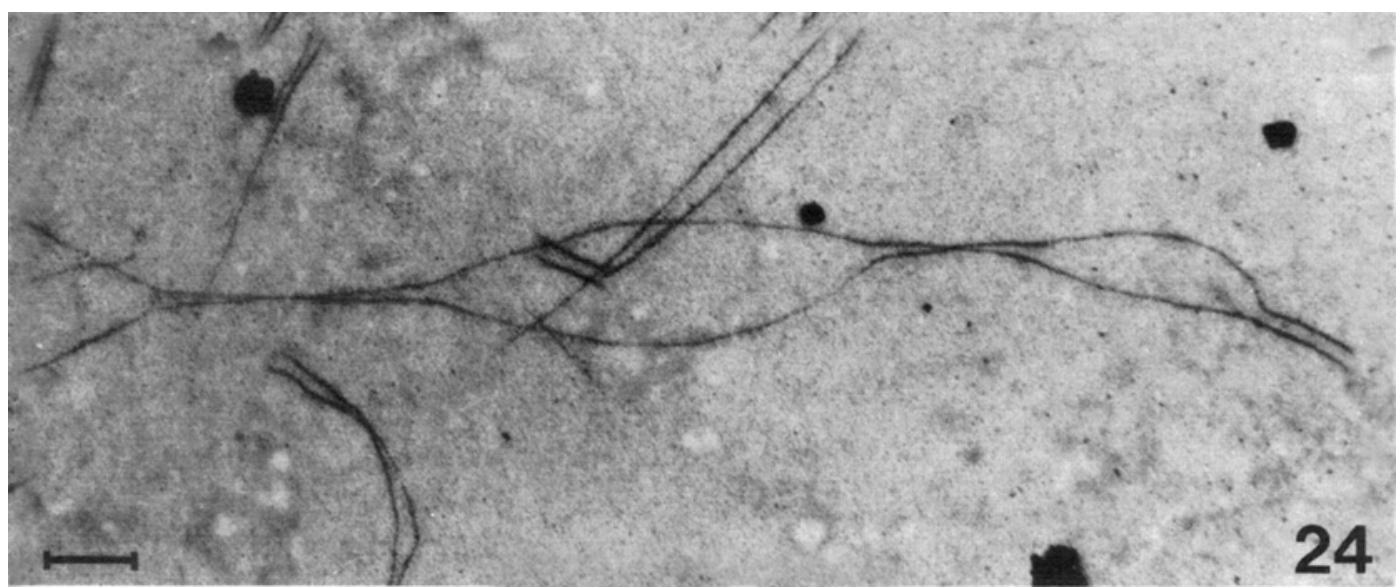

Figure 24. A micrograph of unpaired lateral components in an early diplotene nucleus. A presumptive bivalent interlocking is seen in the middle of the micrograph. (Bar $=1.0 \mu \mathrm{m}$ ).
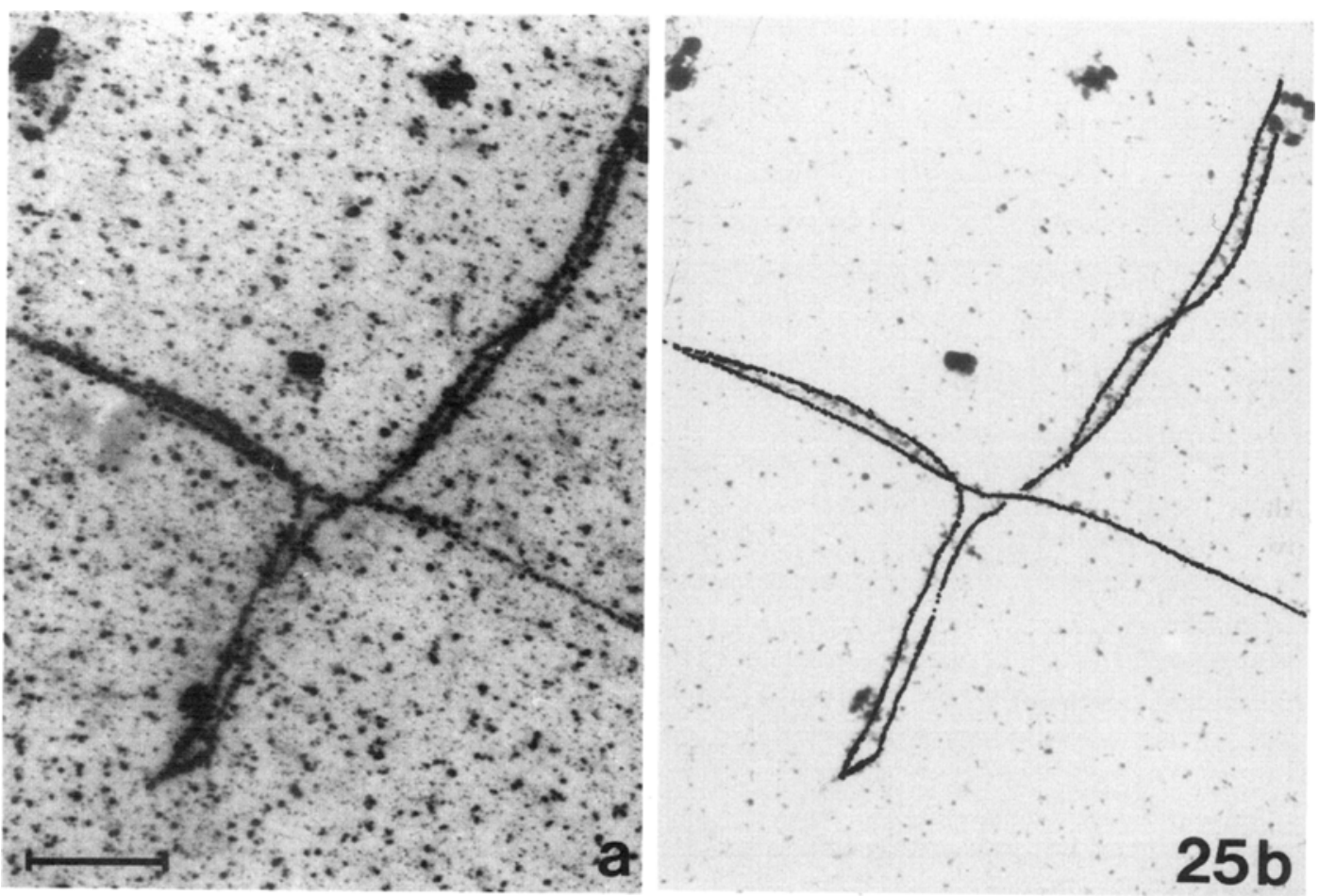

Figure 25. A micrograph and a tracing of a presumptive region of pairing partner exchange from an early diplotene nucleus. $($ Bar $=1.0 \mu \mathrm{m})$. 
Table V.

The number and length of the synaptonemal complex (SC) segments and half the length of unpaired lateral components (LC) in 21 mid diplotene nuclei.

s.d., standard deviation

\begin{tabular}{|c|c|c|c|}
\hline $\begin{array}{l}\text { Nucleus } \\
\text { number }\end{array}$ & $\begin{array}{l}\text { Number of } \\
\text { SC segments }\end{array}$ & $\begin{array}{l}\text { SC length } \\
(\mu \mathrm{m})\end{array}$ & $\begin{array}{l}1 / 2 \text { unpaired } \\
\text { LC length } \\
(\mu \mathrm{m})\end{array}$ \\
\hline MD I & 144 & 653 & 13 \\
\hline MD 2 & 91 & 675 & 19 \\
\hline MD 3 & 109 & 799 & 62 \\
\hline MD 4 & 104 & 604 & 0 \\
\hline MD 5 & 102 & 786 & 36 \\
\hline MD 6 & 126 & 762 & 9 \\
\hline MD 7 & 116 & 625 & 0 \\
\hline MD 8 & 112 & 486 & 0 \\
\hline MD 9 & 130 & 433 & 151 \\
\hline MD 10 & 114 & 394 & 0 \\
\hline MD 11 & 112 & 628 & 18 \\
\hline MD 12 & 100 & 749 & 10 \\
\hline MD 13 & 151 & 808 & 179 \\
\hline MD 14 & 112 & 780 & 40 \\
\hline MD 15 & 142 & 767 & 172 \\
\hline MD 16 & 139 & 740 & 41 \\
\hline MD 17 & 147 & 473 & 30 \\
\hline MD 18 & 88 & 518 & 0 \\
\hline MD 19 & 136 & 450 & 15 \\
\hline MD 20 & 128 & 707 & 0 \\
\hline MD 21 & 85 & 759 & 0 \\
\hline Mean & 119 & 647 & 38 \\
\hline \pm s.d. & \pm 20 & \pm 137 & \pm 57 \\
\hline
\end{tabular}

the initiation of crossing over. It is thus of major importance that crossing over does not occur in the multivalents before the correction process is completed. This may be attributed to a temporal delay of crossing over in multiple associations. Alternatively it can be proposed that crossing over cannot occur between partly homologous chromosome segments. Several lines of evidence thus indicate that there is a general suppression of crossing over between homoeologous chromosomes. In wheat a few multivalents persist during pachytene and although multivalents occasionally are seen at metaphase $\mathrm{I}$, their frequency is substantially lower than the frequency of multivalents at pachytene. This indicates a lack of crossing over between homoeologous and nonhomologous chromosomes in these multivalents which therefore can be re- solved into bivalents during diplotene when the synaptonemal complex is degraded. JENKINS (41) has reported similar results and interpretations from a study of allotetraploid Lolium, where a few multivalents were present at pachytene in the $\mathrm{B}$ chromosome containing allotetraploid, while only bivalents and univalents were observed at metaphase I.

The requirement for a high degree of homology between chromosome segments before crossing over is permitted, is well illustrated in recent studies of yeast $(35,48,49)$. In crosses between Saccharomyces cerevisiae and S. carlsbergensis, karyogamy after conjugation is suppressed if the heterokaryon is heterozygous for the karl mutation. In rare cases, however, such crosses yield viable progeny where one or a limited number of chromosomes has been transferred from the 


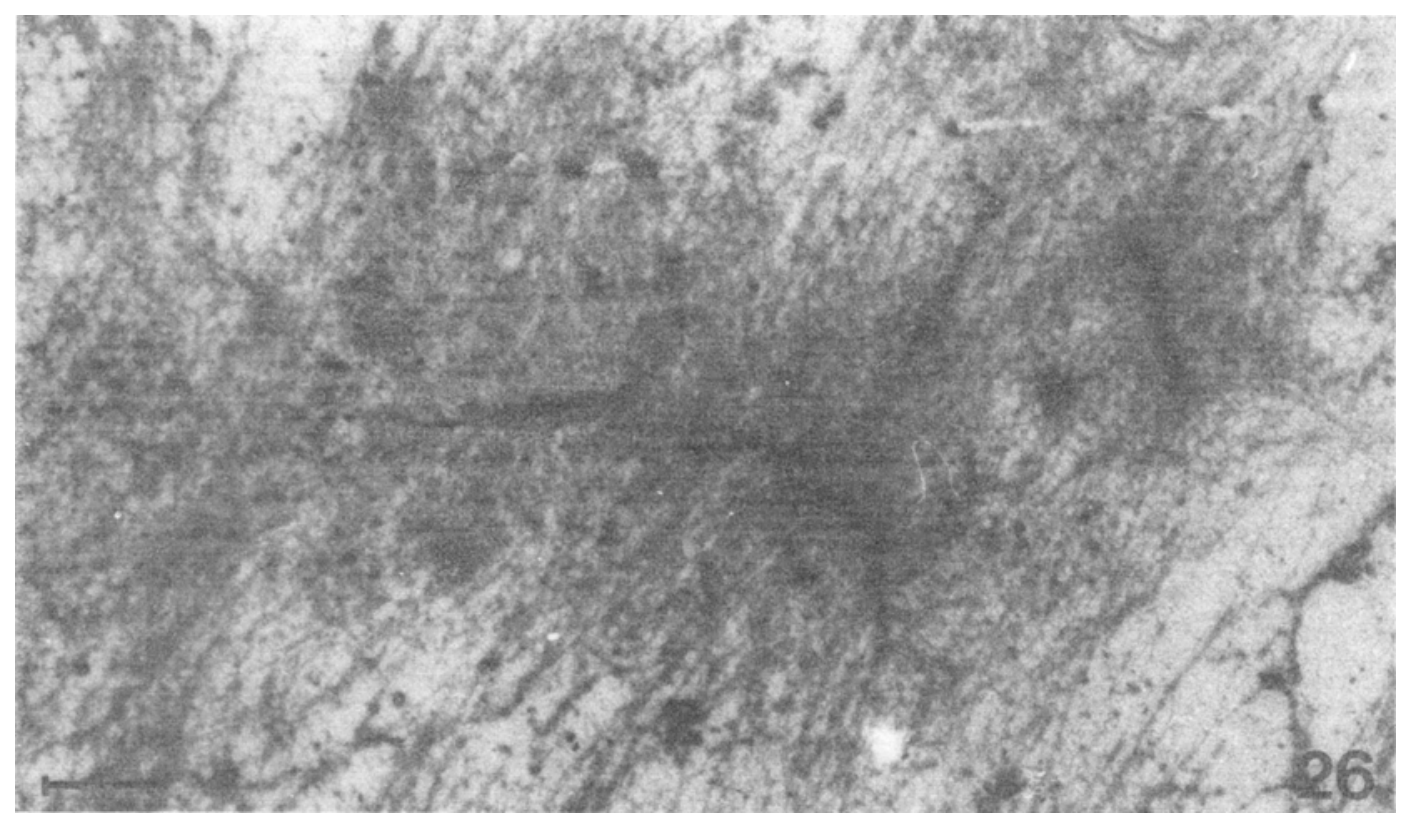

Figure 26. A micrograph of retained synaptonemal complex segments and surrounding chromatin fibers from a mid diplotene nucleus. (Bar $=2.0 \mu \mathrm{m})$.

nucleus of the one parent to the other nucleus. Genetic and molecular studies of a strain of $S$. cerevisiae, where chromosome III has been substituted by its homoeologue from S. carlsbergensis thus revealed that the two chromosomes recombined in a limited region where there was extensive homology, while crossing over was suppressed in segments of partial homology. Although the pairing behaviour of these chromosomes is at present unknown, it is considered likely that they synapse due to their partial homology and the lack of a competing homologue.

Recent biochemical evidence has also suggested that the regulation of crossing over is of importance in ensuring exclusive bivalent formation in allopolyploids $(36,37,63)$. The lily cultivar Black beauty is an achiasmatic diploid hybrid between Lilium speciosum and L. henryi. If pollen mother cells of the hybrid are made allotetraploid by treating the buds with colchicine, chiasma formation occurs and the complement consists at metaphase I of bivalents and a few univalents. Biochemical analysis of the achiasmatic diploid revealed that nicking of the pachytene small nuclear DNA, assumed to relate to crossing over, did not take place, in spite of a normal level of the meiotic endonuclease. This was presumably due to the lack of a pachytene small nuclear RNA, which has to bind to the DNA together with a protein before nicking can occur. The meiotic R-protein which facilitates the annealing of single stranded DNA was only present in one-fifth the amount seen in chiasmatic types. In the allotetraploid, however, the amount of this protein as well as the endonuclease nicking activity were restored to normal levels. Hence, in this case the biochemical machinery required for crossing over is only triggered if there is abundant or perhaps a nearly complete pairing of chromosomes which are homologous. A similar mechanism may account for the lack of crossing over in haploids of wheat, where the three genomes do not recombine as evidenced by the virtual absence of chiasmata at metaphase $I(54,55)$, irrespective of extensive pairing and synaptonemal complex formation (WANG, personal communication). 


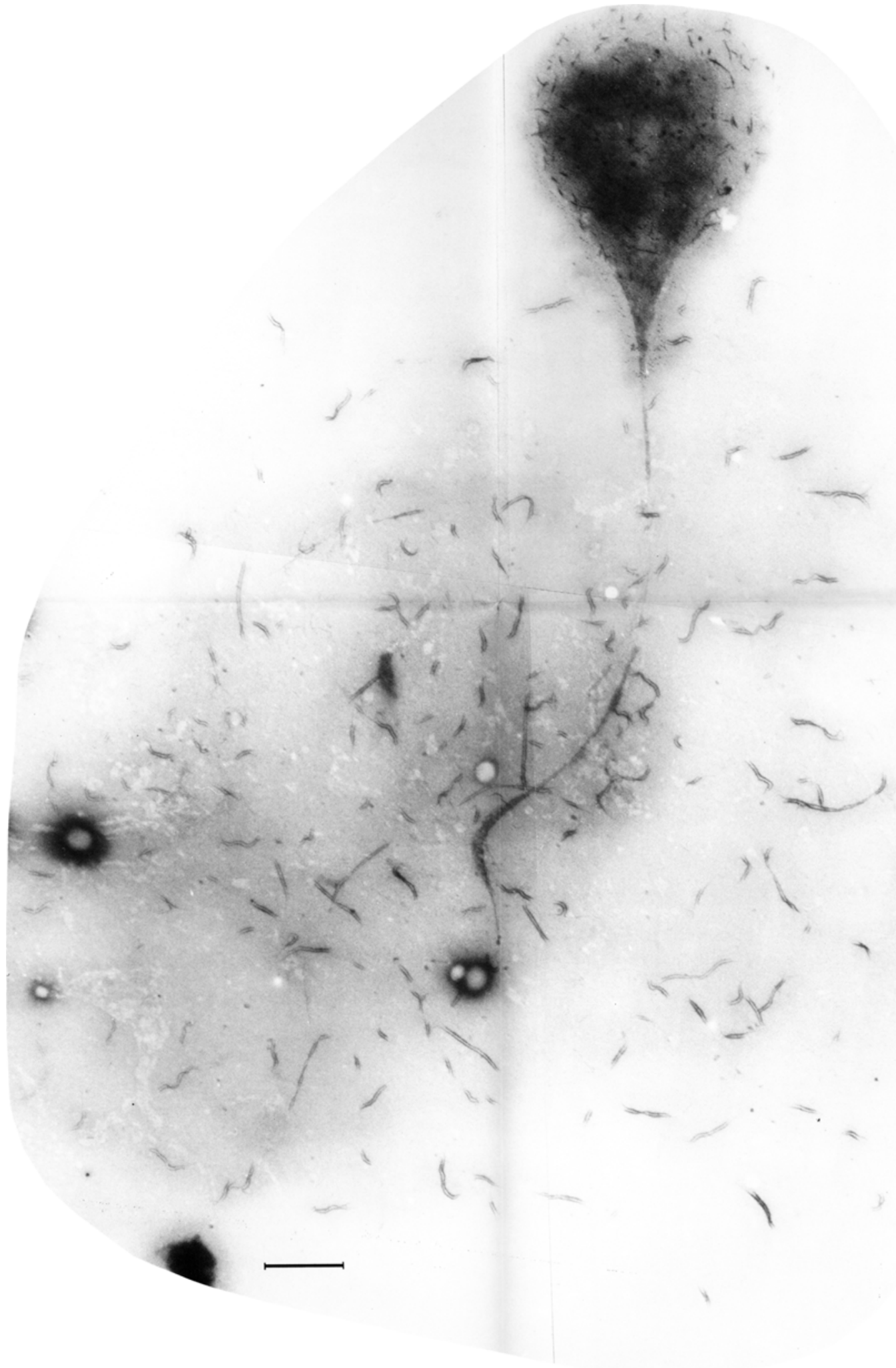




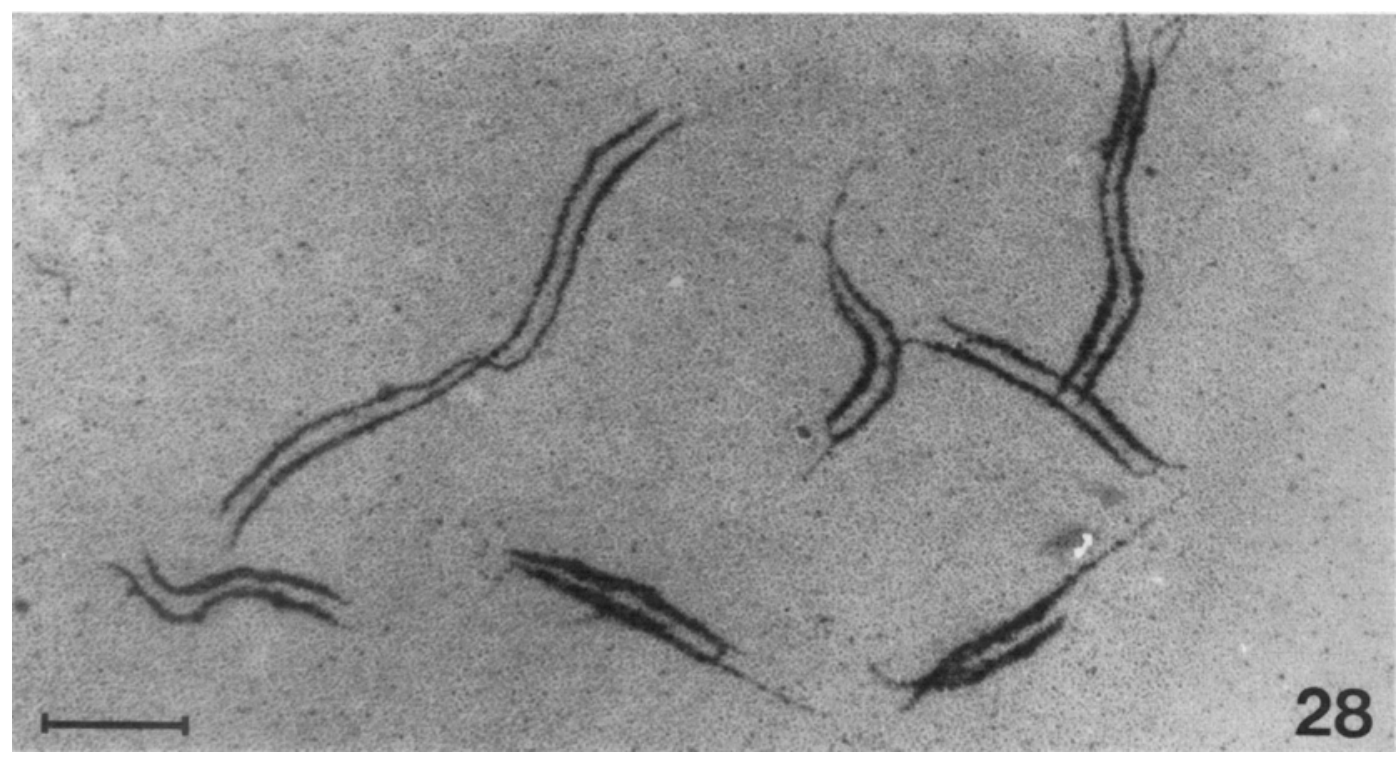

Figure 28. A micrograph of retained synaptonemal complex segments at late diplotene. Note the variation in lateral component thickness. $(\mathrm{Bar}=1.0 \mu \mathrm{m})$.

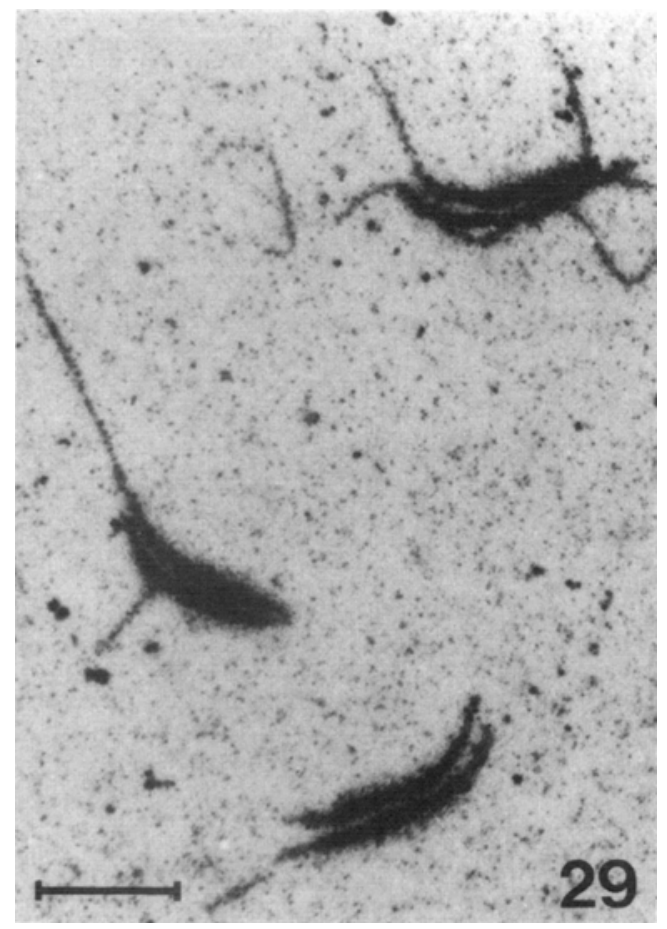

Figure 29. A micrograph of retained synaptonemal complex segments at late diplotene. Note the extensive modification of the lateral components. $($ Bar $=1.0$ $\mu \mathrm{m})$.

\subsection{The effect of the long arm of chromosome 5B}

The present study has suggested that the diploid meiotic behaviour and disomic inheritance of allohexaploid wheat is ensured by the following processes: I) A high stringency of pairing during zygotene which ensures that preferentially homologues pair with a synaptonemal complex. II) A correction process whereby multiple associations are resolved into bivalents. III) A suppression of crossing over between homoeologous chromosomes.

It can at present not be excluded that the $\mathrm{Ph}$ gene(s) on the long arm of chromosome 5B somehow affects aspects of chromosome alignment and synapsis during zygotene. As reported by WISCHMANN (71) an increase in dose from two to four to six copies of the long arm of chromosome 5B leads to increasing asynapsis. On the other hand, the frequency of multivalents at metaphase I in plants nullisomic for chromosome $5 \mathrm{~B}$ is very similar to the frequency of multiple associations seen at late zygotene in the present study, showing that the number of multivalents at metaphase $I$ in nullisomic 5B plants can be accounted for if crossing over 
Table VI.

The number and length of the synaptonemal complex (SC) segments in 16 late diplotene nuclei. s.d., standard deviation

\begin{tabular}{lcc}
\hline $\begin{array}{l}\text { Nucleus } \\
\text { number }\end{array}$ & $\begin{array}{l}\text { Number of } \\
\text { SC segments }\end{array}$ & $\begin{array}{l}\text { SC length } \\
(\mu \mathrm{m})\end{array}$ \\
\hline LD 1 & 156 & 318 \\
LD 2 & 169 & 430 \\
LD 3 & 134 & 376 \\
LD 4 & 119 & 374 \\
LD 5 & 143 & 312 \\
LD 6 & 146 & 342 \\
LD 7 & 129 & 219 \\
LD 8 & 149 & 290 \\
LD 9 & 117 & 210 \\
LD 10 & 125 & 310 \\
LD 11 & 142 & 366 \\
LD 12 & 111 & 181 \\
LD 13 & 116 & 468 \\
LD 14 & 115 & 484 \\
LD 15 & 165 & 290 \\
LD 16 & 144 & 230 \\
\hline Mean & 136 & 325 \\
土 s.d. & \pm 18 & \pm 90 \\
\hline
\end{tabular}

occurs before the transformation of the multiple associations. The proposal by FELDMAN and associates $(5,6,17,18)$ that the $\mathrm{Ph}$ gene(s) control the placement of individual chromosomes in the interphase nucleus, does not appear relevant. According to this hypothesis, homologous and homoeologous chromosomes are closely associated and aligned in the absence of $\mathrm{Ph}$. In normal dosage of $\mathrm{Ph}$ the association of homoeologues is suppressed, while only the alignment of the homologues is maintained. This hypothesis predicts that only homologues pair in normal wheat and interlocking does not occur in the presence of the normal two doses of the $\mathrm{Ph}$ gene. As shown by the present as well as previous ultrastructural studies of wheat this prediction is wrong since multivalent formation and interlocking are regular features of the zygotene chromosome pairing in normal wheat.

\section{ACKNOWLEDGEMENTS}

I am indebted to Professor DITER VON Wettstein, Dr. Søren W. Rasmussen and cand. scient. BENTE WISCHMANN for discussion and review of the manuscript. The expert technical assistance of BIBI STAMPE ANDERSEN, JEAN SAGE and Nina RASMUSSEN is gratefully acknowledged. The Royal Danish Academy of Sciences and Letters are thanked for having provided me with a Niels Bohr Scholarship. The work was also supported by grants BIO-E-417 DK(6) and BI6-168-DK from the Commission of the European Communities.

\section{REFERENCES}

1. ABIRACHED-DARMENCY, M., D.ZICKLER Y. CAUDERON: Synaptonemal complex and recombination nodules in rye (Secale cereale). Chromosoma 88 , 299-306 (1983)

2. Albini. S M.\& G. H. Jones: Synaptonemal complex-associated centromeres and recombination nodules in plant meiocytes prepared by an improved surface-spreading technique. Exp. Cell Res. 155, 588-592 (1984)

3. Albini. S M., G H. Jones \& B M. N. Wallace: A method for preparing two-dimensional surfacespreads of synaptonemal complexes from plant meiocytes for light and electron microscopy. Exp. Cell. Res. 152, 280-285 (1984)

4. Appels, R, W L Gerlach, E. S Dennis, H Swift \& W. J PEACOCK: Molecular and chromosomal organization of DNA sequences coding for the ribosome RNAs in cereals. Chromosoma 78, 293311 (1980)

5. Avivi, L, M. Feldman \& M. Brown: An ordered arrangement of chromosomes in the somatic nucleus of common wheat, Triticum aestivum L. I. Spatial relationships between chromosomes of the same genome. Chromosoma 86, 1-16 (1982)

6. Avivi, L. M. FELDMAN \& M BRown: An ordered arrangement of chromosomes in the somatic nucleus of common wheat, Triticum aestivum L. II. Spatial relationships between chromosomes of different genomes. Chromosoma 86, 17-26 (1982)

7. BAYLISS. M. W \& R RILEY: An analysis of temperature-dependent asynapsis in Triticum aestivum. Genet. Res. Camb. 20, 193-200 (1972)

8. Bloom, S. E. \& C. Goodpasture: An improved technique for selective silver staining of nucleolar organizer region in human chromosomes. Hum. Genet. 34, 199-206 (1976)

9. Bojko, M: Human meiosis VIII. Chromosome pairing and formation of the the synaptonemal complex in oocytes. Carlsberg Res. Commun. 48, $457-483$ (1983) 
10. Bojko. M.: Human meiosis IX. Crossing over and chiasma formation in oocytes. Carlsberg Res. Commur 5 ), 43-72 (1985)

11. Carpenter, A. T. C.: Synaptonemal complex and recombination nodules in wild type Drosophila melanogaster females. Genetics 9?, 511-54! (1979)

12. Catcheside, $\mathrm{D}$ G: The Genetics of Recombination (1977). Edward Arnold, London.

13. Driscoli, C J : Mathematical comparison of homologous and hornoeologous chromosome configurations and the mode of action of the genes regulating paring in wheat. Genetics 92, 947-95 (1979)

14. Driscoll. C. J. L. M. Bielig \& N L. Darvey: An analysis of frequencies of chromosome configurations in wheat and wheat hybrids. Genetics 91 , 755-767 (1979)

15. DVORAK J. K.C. Chen \& B. Giorgi: The C-band pattern of a Ph-mutant of durum wheat. Can. I Gene1. Cytol. 26, 360-363 \{1984)

16. DVORAK. 3 \& K C CMEN Distribuion of nonstructurat variation between wheat cultivars along chromosome 6Bp: Evkdence from the linkage map and physical map of the arm Genetics 106, 325-333 (1984)

17. Feldman, $M$ : The effect of chromosomes $5 B, 5 D$ and $S A$ on chromosomal pairing in Triticum aestrvum. Proc. Nat. Acad. Scr. USA 55, 1447. 1453 (1966)

18. Feldman M \& i. Avivi: Oidered arrangement of chromosomes in wheat. In: Chromosomes Today. eds. M. D. Bennet, A. Gropp \& U. Wolf. 8. 181-189, Georg Allen \& Unwin 1984

19. Gerrard, W I \& R. HANCOCK: Preparation of chromatin from animal tissues and cultured celìs. In: Meihods in Cell Biology, ed. D. M. Prescoti. Vol. XVII. Academic Press, New York, San Fransisco, London 1978

20. Gillies, C. B: The relationship between synaptonemal complexes, recombination nodules and crossing over in Neurospora crassa bivalents and translocation quadrivalents. Genetics 91, 1-17 (1979)

21. GILlies C B.: Vitrastruciural studies of the association of homologous and non-homologous parts of chromosomes in the mid-prophase of meiosis in Zea mays. Maydica 28, 265-287 (1983)

22. Gulues, C B: An electron mirsoscopic study of synaptonemal complex formation at zygotene in rye. Chromosoma 92. 165-175 (1985)

23. Glamann, J : Crossing over in the male mouse as analyzed by recombination nodules and bars. Carlsberg Res. Commun 51 143-162 (1986)

24. GoOdPasture, $C$ \& S E BroOM: Visualization of nucleolar organizer regions in mammalian chromosomes using silver staining. Chromosoma 53 , 37-50 (1975)

25. HASENKAMPF, C. A.: Synaptonemal complex formation in pollen mother cells of Tradescantia. Chromosoma 90, 275-284 (1984)

26. Heslop-HARRISON, J $S$ \& M. D BenNeTt: Interlocked bivalents in reconstructed metaphase I cells of bread wheat. J. Cell Sci. 75, 85-92 (1985)

27. Hовоцтн, P: Chromosome pairing in allohexaploid wheat var. Chinese Spring. Transformation of multivalents into bivalents, a mechanism for exclusively bivalent formation. Carlsberg Res. Commun. 46, 129-173 (1981)

28. Holm, $\mathrm{P}$ B : Three dimensional reconstruction of chromosome pairing during the zygotene stage of meiosis in Lilium longiflorum (Thunb. ). Carlsberg Res. Commun. 42, 103-151 (1977)

29. Holm, P. B : Ultrastructural characterization of meiosis. Biol. Skr. Dan. Vid. Selsk. 25, 39-90 (1985)

30. Holm. P. B. \& S. W. Rasmussen: Chromosome pairing, recombination nodules and chiasma formation in diploid Bombyx males. Carlsberg Res Commun. 45, 483-548 (1980)

31. Holm, P B. \& S Rasmussen: Human meiosis VI. Crossing over in human spermatocytes. Carlsberg Res. Commun. 48, 385-413 (1.983)

32. Holm. P. B. \& S W Rasmussen: Human meiosis VII. Chiasma formation in human spernatocytes. Carlsberg Res. Commun. 48, 415-456 (1983)

33. Holm P B.\&S W. Rasmussen: The synaptonemal complex in chromosome pairing and disjunction. In: Chromosomes Today, ed. M. D. Bennett, A. Gropp and U. Wolf. 8, 104-116 Georg Allen \& Unwin 1984

34. Holm, P B, S W. Rasmussen, D.Zickler. B. C. Lu \& J SAGE: Chromosome pairing, recombination nodules and chiasma formation in the basidiomycete Coprinus cinereus. Carlsberg Res. Commun. 46, 305-346 (1981)

35. HolmberG, S: Genetic differences between Saccharomyces carlsbergensis and $\mathrm{S}$. cerevisjae II. Restriction endonuclease analysis of genes in chromosome 11I. Carlsberg Res. Commun. 47, 233-244 (1982)

36. HotTa, Y, M D Bennett. L. A TOledo \& H STERN: Regulaion of $R$ proiein and endonuclease actrvities in meiocytes by homologous chromo some paining. Chromosoma, 72, 191-20l (1979)

37. HoTTA, Y \& H STERN: Small nuclear RNA molecules that regulate nuclease accessibility in specific chromatin regions of meiotic cells. Cell 27. 309-319 (1981)

38. JENkins, G.: Chromosome paing in Triucum 
aestivum cv. Chinese Spring. Carlisberg Res. Commun. 48. 255-283 (1983)

39. JENKINS, G: Synaptonemal complex formation in hybrids of Lolium temulentum $\times$ Lolium perenne (L. ). 1. High chiasma frequency diploid. Chromosoma 92, 81-88 (1985)

40. JENKINS G : Synaptonemal complex formation in hybrids of Lolium temulentum $\times$ Lolium perenne (L. ). Il. Triploid. Chromosoma 92, 387-390(1985)

4: JENKINS, G : Synaptonemal complex formation in hybrids of Lolium temulentum $\times$ Lolium perenne (L. ). III. Tetraploıd. Chromosoma (in press)

42. KOEBNER, R M D \& K.W SHEPHERD: Induction or recombination between rye chromosome $1 R L$ and wheat chromosomes. Theor. Appl. Genet 71 , $208-215(1985)$

4\%. LOIDL. J \& G H JONES: Synaptonemal compieX spreading in Allium : Triploid A. sphaerocephalum. Chromosoma (in press)

44 Mclntosh, R A : A catalogue of gene symbols for wheat (1983 edition). Proc. 6th International Whea: Genetics Symposium 1 197-1254 (1983)

45. MilLER. T E: The homoeologous relationship between the chromosomes of rye and wheat. Current: status. Can 1. Genet. Cytol. 25, 578-589

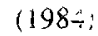

46. MOSES. Mi j : Synaptonemal complex karyotyping in spermatocytes of Chinese hamster (Cricetulus griseus). i. Morphology of the autosomal compiement in spread preparations. Chromosoma 60, 99-125(197\%)

47. NOSES. Mi J \& A J SOlARt: Positive contrast staining and protected drying of surface spreads: Electron microscopy of the synaptonemal complex by a new metnod. J. Ultrast. Res. 54, 109-1 14 (1976)

48 Nilsson-Tillgren $T$. J G Litske Petersen, $S$. HOLMBERG\& Mi C KIELLAND-BRANDT: Transfer of chromosome III during kar mediated cytoduction in yeast. Carisberg Res Commun. 45, 113-117 (1980)

49. Nilsson-Tillgren, T, C Gjermansen, M C KielLAND-Brand, j $G$ Litske Petersen \& S. HolmBERG: Genetic differences between Saccharomyces carisbergensis and S. cerevisiae. Analysis of chromosome UII by single chromosome transfer. Carlsherg Kes. Commun. 46. 65-76 (1981)

50. Ok+moro, $\mathrm{N}$. Asynaptic effect on chromosome V. Wheas in Serv 5.6-7(1957)

51. R+SMLISSEN, $5 \mathrm{~W}$. The transformation of the synaptonema! complex into tî̉e "elimination chromatun" in Bombyx mori oocytes. Chromosoma 60. 205-22: 1977

52. RASMUSSEN, S a P B HoLm: Human meiosis 11. Chromosome painng and recombination nodules in numan spermatocyses. Carlsberg Res. Com- mun. 43, 275-327 (1978)

53. Rasmussen, S W \& P B Holm: Chromosome pairing in autotetraploid Bombyx females. Mechanism for exclusive bivalent formation. Carlsberg Res. Commun. 44, 101-125 (1979)

54. RHLE, R: The diploidisation of polyploid wheat. Heredity $15,407-429$ (1960)

55. RIEEY. R \& V CHAPMAN: Genetic control of the cytologically diploid behaviour of hexaplord wheat. Nature 182.713-715 (1958)

56. Riley, R, V Chapman. R. M. Young \& A. M BELFIELD: Control of meiotic chromosome painng by the chromosomes of homoeologous group 5 of Triticum aestivum. Nature 212, 1475-1477(1966)

57. RILEY. R \& C KEMPANNA: The homoeologous nature of the non-homologous meiotic painng in Triticum aestivum deficient for chromosome $\mathrm{V}$ (5B). Heredity 18, 287-306 (1963)

58. SEARS. E R.: Wheat cytogenetics. Ann. Rev. Genet. 3, 451-468 (1969)

59. SEARS, E R : Genetic control of chromosome parring in wheat. Ann. Rev. Genet. 10, 31-51 (1976)

60 SEARS, E R: An induced mutant with homoeologous pairing in common wheat. Caa. J. Genet. Cytol 19,585-593 (1977)

61. SEARS, E R: The transfer to wheat of interstitia: segments of alien chromosomes. Proc. oth International Wheat Genetics Symposium, 5-12 (1983)

62. SOLARI, A I: The behavior of chromosomal axes during diplotene in mouse spermatocytes. Chromosoma 31, 217-230 (1970)

63. TOLEDo. L.A, M D BenNetT \& H STERN: Cytoingical investigation of the effect of colchicine on meiosis in Lilium hybrid cv. "Black Beauty" microsporocytes. Chromosoma 72, 157-173 (1979)

64. WALl, A M.. R. RILEY \& V CHAPMAN: Wheat mutants permitting homoeologous meiotic chromosome pairing. Genet. Res. Camb. 18, 311-328 (1971)

65. WALL, A M , R. RILEY \& M. D GALE: The position of a locus on chromosome $5 \mathrm{~B}$ of Triticum aestivum affecting homoeologous meiotic pairing. Genet. Res. Camb. 18, 329-339 (1971)

66. Watanabe, K.: Studies on the control of diploidlike meiosis in polyplold taxa of Chrysantinemur I. Hexaploid Ch. japonense Nakai. Cytologia 46, 459-498 (1981)

67. WESTERGAARD, M. \& D VON WETTSTEIN: The meiotic cycle in an ascomycete. In effects of radiation on meiotic systems. Internat. Atom. Energy Agency. Panel Proc. Sci. Pub. 173, 1 13-12! (1968)

68. WestergaARD, M \& D vON WetTstein: Studies on the mechanism of crossing-over $\mathrm{V} V$. The molecular organization of the synaptonemal compiex $1 n$ Neottiella (Cooke) Saccardo Ascomycetes). 
Compt. Rend. Trav. Lab. Carlsberg 37, 239-268 (1970)

69. Westergaard, M. \& D von Wettstein: The synaptinemal complex. Ann. Rev. Genet. 6, 71110 (1972)

70. WetTSTEIN,D von, S. W. RaSMUSSEN \& P. B. HOlm: The synaptonemal complex in genetic segregation. Ann. Rev. Genet. 18, 331-431 (1984)

71. WischmanN, B : Chromosome pairing and chiasma formation in wheat plants triisosomic for the long arm of chromosome 5B. Carlsberg Res. Commun. 51, 1-25 (1986)

72. YaCoBi, Y Z, T Mello-Sampayo \& M Feldman: Genetic induction of bivalent interlocking in common wheat. Chromosoma 87, 165-175 (1982)

73. ZICKLER, D: Development of the synaptonemal complex and the "recombination nodules" during meiotic prophase in the seven bivalents of the fungus Sordaria macrospora Auersw. Chromosoma 61, 289-316 (1977)

Accepted by SO ANDERSEN 